\title{
Aspectos estatísticos da amostragem de água de lastro
}

\author{
Eliardo Guimarães da Costa
}

DissertaÇÃo APRESENTADA

$\mathrm{AO}$

Instituto de Matemática e EstatísticA

DA

Universidade DE SÃo Paulo

PARA

OBTENÇÃO DO TÍTULO

DE

Mestre EM Cî̂nCIAS

\author{
Programa: Estatística \\ Orientador: Prof. Dr. Julio da Motta Singer
}

Durante o desenvolvimento deste trabalho o autor recebeu auxílio financeiro do CNPq

São Paulo, março de 2013 


\section{Aspectos estatísticos da amostragem de água de lastro}

Esta versão da dissertação contém as correções e alterações sugeridas pela Comissão Julgadora durante a defesa da versão original do trabalho, realizada em 01/03/2013. Uma cópia da versão original está disponível no Instituto de Matemática e Estatística da Universidade de São Paulo.

Comissão Julgadora:

- Prof. Dr. Julio da Motta Singer (orientador) - IME-USP

- Prof. Dr. Rubens Mendes Lopes - IO-USP

- Profa. Dra . Linda Lee Ho - EP-USP 
"O universal pelo regional."

Lema da Universidade Federal do Ceará

"I am the passenger and $I$ ride and I ride

I ride through the city's backsides I see the stars come out of the sky

Yeah the bright and hollow sky You know it looks so good tonight

I am the passenger I stay under glass

I look through my window so bright

I see the stars come out tonight

I see the bright and hollow sky

Over the city's ripped sky And everything looks good tonight(...)

He sees the city's ripped backsides He sees the winding ocean drive And everything was made for you and me All of it was made for you and me 'Cause it just belongs to you and me So let's take a ride and see what's mine(...)"

The Passenger - Iggy Pop

"Fortitudine: força, valor e coragem." Lema da cidade de Fortaleza 


\section{Agradecimentos}

Gostaria de agradecer:

Aos meus pais, Sônia e Sérgio, o carinho, a educação, o incentivo, o apoio mesmo sabendo que eu estaria longe. Aos meus irmãos, Eduardo e Sara, os momentos juntos, as alegrias, as risadas etc, e aos meus familiares que de alguma forma contribuíram para minha formação pessoal e profissional.

À minha namorada, Rachel Tarini, a companhia, o carinho, o apoio mesmo sabendo que estaríamos longe um do outro, os momentos juntos, os sonhos etc $(-\log 0)$.

Ao meu orientador, professor Julio Singer, a oportunidade, a paciência, os ensinamentos etc.

Aos membros da banca examinadora as sugestões e comentários sobre o trabalho, em especial ao professor Rubens a oportunidade de participar do projeto sobre água de lastro.

Aos professores do IME-USP: Luis Gustavo, Anatoli Iambartsev, Gilberto Paula, Heleno Bolfarine e Florência Leonardi que contribuíram para minha formação durante o mestrado.

Aos amigos do IME-USP os momentos de descontração, o futebol, as pizzas, os churrascos etc, dentre os quais destaco: Agatha, Andrés (Arias), Andrés (Rosso), Aníbal, Brian (Garoto), Benigno (Ó paí ó), Bruno (Quixadá, parceiro na "chegada" em SP), Carlos, Daniel, Diana (Colômbia), Diego (Chile), Elisângela (PB), Elizabeth (Colômbia), Fabi(ã)o, Felipe (Bhering), Isabelie (México), Josemir (PB), Leandr(ã)o, Marci(ã)o, Marcelo, Mel, Michel, Nathália, Rafael (o Jovem), Rafael Paixão, Raony, Soane, Thiago (Bayesiano), Tiago (Palmeirense), Vinicius (Cokin), William (Colômbia).

Aos amigos da república Gobbo os momentos de descontração, os churrascos: Alan (Peru), Benigno (Ó paí ó), Brian (Peru), Bruno (Quixadá), Daniel (México), Felipe (Santos), Inés (Camarões), Josué, Marcelo (Brasília), Moises (Guiné-Bissau), Othon (aka "Othin"), Tom (Quixeramobim), Rennan.

Aos professores do DEMA-UFC os ensinamentos, dentre os quais destaco: Ailton, André Jalles, César Teixeira, Lassance, Rafael Castro, Robson, Silvia, Welliandre. Em especial aos professores Maurício e Juvêncio a paciência, ensinamentos, incentivo para prosseguir para o mestrado.

Aos amigos da Estatística-UFC os momentos de descontração, companherismo etc, dentre os quais destaco: Andréa, Bruno (Quixadá), David (Lipan), Dayse, Ellyson, Helton (Jaguar), Jéssica, Jhonny, Josian, Josy, Laura, Leandro (Cabo Verde), Lívia, Patrícia, Rafa(el), Ramsés, Raquel, Sarah, Thiago (Minhoca).

Aos professores da escola Hilza Diogo de Oliveira (Fortaleza, CE) que contribuíram de alguma forma na minha formação, em especial a professora Socorro Oliveira (Matemática) por acreditar no meu potencial, o incentivo, os conselhos; lembro que a senhora me emprestou uma edição da 
RPM (editada no IME-USP) e quem diria que eu iria vir estudar no IME-USP.

Ao CNPq o apoio financeiro. 


\section{Resumo}

COSTA, E. G. Aspectos estatísticos da amostragem de água de lastro. 2013. 79 f. Dissertação (Mestrado) - Instituto de Matemática e Estatística, Universidade de São Paulo, São Paulo, 2013.

A água de lastro de navios é um dos principais agentes dispersivos de organismos nocivos à saúde humana e ao meio ambiente e normas internacionais exigem que a concentração desses organismos no tanque seja menor que um valor previamente especificado. Por limitações de tempo e custo, esse controle requer o uso de amostragem. Sob a hipótese de que a concentração desses organismos no tanque é homogênea, vários autores têm utilizado a distribuição Poisson para a tomada de decisão com base num teste de hipóteses. Como essa proposta é pouco realista, estendemos os resultados para casos em que a concentração de organismos no tanque é heterogênea utilizando estratificação, processos de Poisson não-homogêneos ou assumindo que ela obedece a uma distribuição Gama, que induz uma distribuição Binomial Negativa para o número de organismos amostrados. Além disso, propomos uma nova abordagem para o problema por meio de técnicas de estimação baseadas na distribuição Binomial Negativa. Para fins de aplicação, implementamos rotinas computacionais no software R.

Palavras-chave: determinação de tamanho amostral, distribuição Poisson, distribuição Binomial Negativa, processos de Poisson não-homogêneos. 


\section{Abstract}

COSTA, E. G. Statistical aspects of ballast water sampling. 2013. 79 f. MSc thesis - Instituto de Matemática e Estatística, Universidade de São Paulo, São Paulo, 2013.

Ballast water is a leading dispersing agent of harmful organisms to human health and to the environment and international standards require that the concentration of these organisms in the tank must be less than a prespecified value. Because of time and cost limitations, this inspection requires the use of sampling. Under the assumption of an homogeneous organism concentration in the tank, several authors have used the Poisson distribution for decision making based on hypothesis testing. Since this proposal is unrealistic, we extend the results for cases in which the organism concentration in the tank is heterogeneous, using stratification, nonhomogeneous Poisson processes or assuming that it follows a Gamma distribution, which induces a Negative Binomial distribution for the number of sampled organisms. Furthermore, we propose a novel approach to the problem through estimation techniques based on the Negative Binomial distribution. For practical applications, we implemented computational routines using the $\mathrm{R}$ software.

Keywords: sample size determination, Poisson distribution, Negative Binomial distribution, nonhomogeneous Poisson processes. 


\section{Sumário}

Lista de Figuras $\quad$ xi

Lista de Tabelas $\quad$ xiii

1 Introdução $\quad 1$

2 Avaliação do cumprimento das normas IMO por meio de testes de hipóteses 5

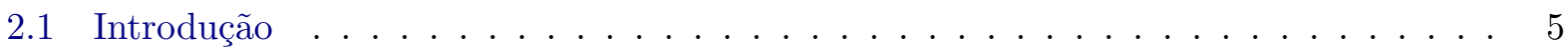

2.2 Amostragem com concentração de organismos homogênea . . . . . . . . . . . . . 5

2.3 Amostragem com concentração de organismos heterogênea . . . . . . . . . . . . . . 9

2.4 Amostragem com concentração de organismos aleatória . . . . . . . . . . . . . . . 19

3 Avaliação do cumprimento das normas IMO por meio de estimação 25

3.1 Introdução . . . . . . . . . . . . . . . . . . . . . . 25

3.2 Tamanho amostral aproximado . . . . . . . . . . . . . . . 26

3.3 Tamanho amostral exato . . . . . . . . . . . . . . . . . . 27

3.3.1 Fixação de limite apenas para o erro absoluto . . . . . . . . . . . . . . 28

3.3.2 Fixação de limite apenas para o erro relativo . . . . . . . . . . . . . . 29

3.3.3 Fixação de limites para os erros abosoluto e relativo . . . . . . . . . . . . . . 29

4 Conclusões e sugestões para futuras pesquisas $\quad 41$

A Demonstrações do Teorema 3.2 e do Corolário $3.1 \quad 43$

A.1 Demonstração do Teorema $3.2 \ldots \ldots \ldots$. . . . . . . . . . . . . 43

A.2 Demonstração do Corolário $3.1 \ldots \ldots \ldots$. . . . . . . . . . . . . . . 47

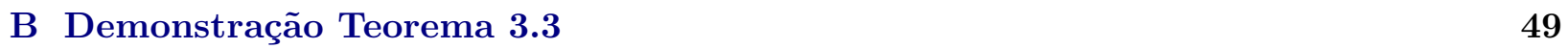

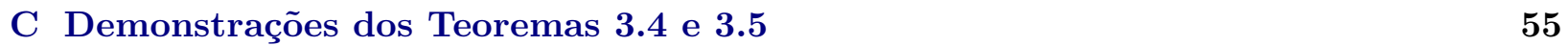

C.1 Demonstração do Teorema $3.4 \ldots \ldots \ldots \ldots \ldots \ldots$

C.2 Demonstração do Teorema $3.5 \ldots \ldots \ldots \ldots \ldots \ldots \ldots$

$\begin{array}{ll}\text { D Pseudo-algoritmos } & \mathbf{5 9}\end{array}$

D.1 Tamanho amostral para o teste $(2.4) \ldots \ldots \ldots \ldots \ldots$. . . . . . . . 59

D.2 Tamanho amostral para o teste $(2.7) \ldots \ldots \ldots \ldots \ldots \ldots \ldots$

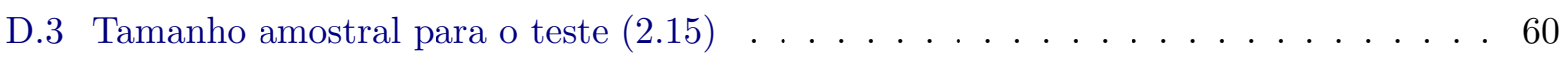

D.4 Tamanho amostral exato para o Teorema $3.3 \ldots \ldots \ldots \ldots$ 


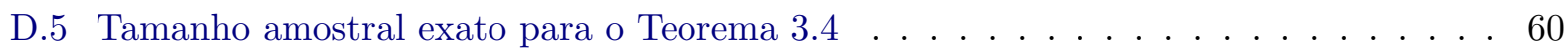

D.6 Tamanho amostral exato para o Teorema $3.5 \ldots \ldots \ldots \ldots \ldots$

$\begin{array}{ll}\text { Referências } & 61\end{array}$ 


\section{Lista de Figuras}

1.1 Navio descarregando água de lastro que possui organismos viáveis. . . . . . . . . . . 2

1.2 Navio descarregando água de lastro. . . . . . . . . . . . . . . . . . 3

2.1 Poder para o teste com uma alíquota usando a distribuição Poisson . . . . . . . . . . 7

2.2 Função da concentração de organismos na forma de potência $(2.10)$. . . . . . . . . 13

2.3 Simulação da contagem de organismos usando a função potência (2.10) em que $\theta<1.14$

2.4 Simulação da contagem de organismos usando a função potência (2.10) em que $\theta=1$. 15

2.5 Simulação da contagem de organismos usando a função potência $(2.10)(1<\theta<2)$. 16

2.6 Simulação da contagem de organismos usando a função potência (2.10) em que $\theta=2.17$

2.7 Simulação da contagem de organismos usando a função potência (2.10) em que $\theta>2$. 18

2.8 Função da concentração de organismos na forma recíproca $(2.11) \ldots$. . . . . . . . 19

2.9 Simulação da contagem de organismos usando a função recíproca (2.11) . . . . . . . . 20

2.10 Algumas formas para a distribuição da concentração de organismos no tanque de lastro. 21

2.11 Binomiais Negativas com média $\lambda=10$, Poisson com média $\lambda=10$. . . . . . . . 23

3.1 Estimativas e intervalos de confiança de $\lambda_{i}$ e $k(n=803) \ldots \ldots \ldots \ldots$

3.2 Distribuições de probabilidade teóricas e ajustadas $(\lambda=3, k=1) \ldots \ldots \ldots$. . . . 34

3.3 Estimativas e intervalos de confiança de $\lambda_{i}$ e $k(\lambda=5, k=20$ e $n=44) \ldots \ldots 35$

3.4 Distribuições de probabilidade e ajustadas $(\lambda=5, k=20) \ldots \ldots \ldots \ldots$

3.5 Estimativas e intervalos de confiança de $\lambda_{i}$ e $k(\lambda=18, k=3$ e $n=278) . \ldots 36$

3.6 Distribuições de probabilidade e ajustadas $(\lambda=18, k=3) \ldots \ldots \ldots$. . . . . 37

3.7 Estimativas e intervalos de confiança de $\lambda_{i}$ e $k(\lambda=13, k=15$ e $n=86) . \ldots 37$

3.8 Distribuições de probabilidade e ajustadas $(\lambda=13, k=15) \ldots \ldots \ldots$

3.9 Estimativas e intervalos de confiança de $\lambda_{i}$ e $k(\lambda=20, k=350$ e $n=24) \ldots \ldots . .39$

3.10 Distribuições de probabilidade e ajustadas $(\lambda=20, k=350) \ldots \ldots \ldots$ 


\section{Lista de Tabelas}

1.1 Volume de lastro nos tanques de alguns navios no porto de Tubarão/ES . . . . . . . 4

1.2 Taxa de bombeamento para alguns tipos de embarcação. . . . . . . . . . . . . . . 4

2.1 Limites de descumprimento da norma IMO usando a distribuição Poisson . . . . . . 6

2.2 Menor número de alíquotas $(n)$ para o teste (2.4) usando a distribuição Poisson . . . 8

2.3 Contagens simuladas de organismos usando (2.6) $\operatorname{com} n=13, \lambda=13$ e $v=1 \mathrm{~m}^{3}$. . 8

2.4 Menores volumes amostrais $\left(v_{h}\right)$ para o qual o poder do teste $(2.7)$ é $\geq 1-b(b=0.05) 10$

2.5 Menores volumes amostrais $\left(v_{h}\right)$ para o qual o poder do teste $(2.7)$ é $\geq 1-b(b=0.10) 10$

2.6 Contagens simuladas de organismos usando a função potência $(2.10)$ em que $\theta<1$. . 12

2.7 Estimativas para o modelo (2.9) usando a função potência $(2.10)$ em que $\theta<1 \ldots 13$

2.8 Contagens simuladas de organismos usando a função potência (2.10) em que $\theta=1$. . 14

2.9 Estimativas para o modelo (2.9) usando a função potência $(2.10)$ em que $\theta=1$. . 14

2.10 Contagens simuladas de organismos usando a função potência $(2.10)(1<\theta<2)$. . 15

2.11 Estimativas para o modelo (2.9) usando a função potência (2.10) em que $1<\theta<2$. 15

2.12 Contagens simuladas de organismos usando a função potência (2.10) em que $\theta=2$. . 16

2.13 Estimativas para o modelo (2.9) usando a função potência $(2.10)$ em que $\theta=2 \ldots 16$

2.14 Contagens simuladas de organismos usando a função potência $(2.10)$ em que $\theta>2$. . 17

2.15 Estimativas para o modelo (2.9) usando a função potência (2.10) em que $\theta>2 \ldots 17$

2.16 Contagens simuladas para o modelo (2.9) usando a função recíproca (2.11) . . . . . 18

2.17 Estimativas para o modelo (2.9) usando a função recíproca $(2.11)$. . . . . . . . . 19

2.18 Menor número de alíquotas de $1 \mathrm{~m}^{3}(n)$ usando a Binomial Negativa com $k=1$. . 22

2.19 Menor número de alíquotas de $1 \mathrm{~m}^{3}(n)$ usando a Binomial Negativa com $k=3$. . . 22

2.20 Menor número de alíquotas de $1 \mathrm{~m}^{3}(n)$ usando a Binomial Negativa com $k=25$. . 22

2.21 Contagens de organismos simuladas usando (2.14) para $\lambda=14, k=3$ e $n=33$. . . 23

2.22 Contagens de organismos simuladas usando (2.14) para $\lambda=14, k=25$ e $n=11$. . 23

2.23 Contagens de organismos simuladas usando (2.14) para $\lambda=15, k=3$ e $n=23 . .24$

3.1 Tamanho amostral aproximado usando o Teorema $3.2(\delta=0.05) \ldots \ldots \ldots \ldots$. . . . 27

3.2 Tamanho amostral aproximado usando o Teorema $3.2(\delta=0.01) \ldots \ldots \ldots$

3.3 Limitantes $a$ e $b$ da média para o cálculo do tamanho amostral exato. . . . . . . . 30

3.4 Tamanho amostral exato usando o Teorema $3.3 \ldots \ldots \ldots$. . . . . . . . . . 30

3.5 Tamanho amostral exato usando o Teorema $3.4 \ldots \ldots \ldots$. . . . . . . . . 31

3.6 Tamanho amostral exato usando o Teorema $3.5(\delta=0.05) \ldots \ldots \ldots \ldots$

3.7 Tamanho amostral exato usando o Teorema $3.5(\delta=0.01) \ldots \ldots \ldots$. . . . . . 32

3.8 Estimativas para o modelo (2.14) com média $\lambda_{i}, k=1$ e $n=803 \ldots \ldots$. . . . . 33 
3.9 Estimativas para o modelo (2.14) com média $\lambda_{i}, k=20$ e $n=44 \ldots \ldots$. . . . . 35

3.10 Estimativas para o modelo (2.14) com média $\lambda_{i}, k=3$ e $n=278 \ldots \ldots$. . . . . . 35

3.11 Estimativas para o modelo (2.14) com média $\lambda_{i}, k=15$ e $n=86 \ldots \ldots$. . . . . 35

3.12 Estimativas para o modelo (2.14) com média $\lambda_{i}, k=350$ e $n=24 \ldots \ldots$. . . . . 38 


\section{Capítulo 1}

\section{Introdução}

Lastro é qualquer material usado para aumentar o peso e/ou manter a estabilidade de um objeto. Navios cargueiros utilizam lastro para navegar com segurança quando estão sem ou com pouca carga, mantendo suas hélices propulsoras submersas. Areia, madeiras e rochas eram usados para essa finalidade até o início do século XX. Com o aumento do tamanho dos navios, o processo de lastragem com sólidos tornou-se inadequado [Carmo (2008)] e a água captada de rios ou mares passou a ser utilizada como lastro devido à facilidade de manejo, dando origem ao termo água de lastro. A facilidade daí oriunda, tem um preço, pois a biota liberada com a descarga no destino pode alterar o ecossistema aquático local [Souza et al. (2001); Walton (1998)]. Organismos introduzidos por navios têm prejudicado ambientes naturais, a saúde humana e a economia [David et al. (2007)]. Com o crescimento do tráfego marítimo e do uso de grandes navios, a água de lastro tornou-se o mais perigoso agente de dispersão de organismos marinhos [Souza et al. (2001)]. Tais organismos têm sido registrados em Santos/SP, Porto Alegre/RS, no Pantanal, na Bacia Amazônica e em Salvador/BA [Beasley et al. (2003); Carqueija \& Gouvêa (1996); Hager (2008); Tavares \& Mendonça Jr. (2004)]. Segundo Ruiz et al. (2000) patógenos humanos também estão sendo transferidos através de água de lastro. Como exemplo, podemos citar uma epidemia de cólera ocorrida no Peru em 1991 e rapidamente disseminada pela América Latina [McCarthy et al. (1992)].

Em torno de 12 bilhões de toneladas de água de lastro são transportadas anualmente em todo o mundo [IMO/MEPC (1998)], com cerca de 7000 espécies transitando diariamente [Carlton (2001)]. No Brasil, a situação é complicada devido à sua extensa costa e ao comércio exterior do país por via marítima que corresponde a 95\% do total [Carmo (2008)]. A Organização Marítima Internacional (IMO, na sigla em inglês) estimou a quantidade de água de lastro movimentada na costa brasileira em 40 milhões de toneladas por ano [Souza et al. (2001)]. 


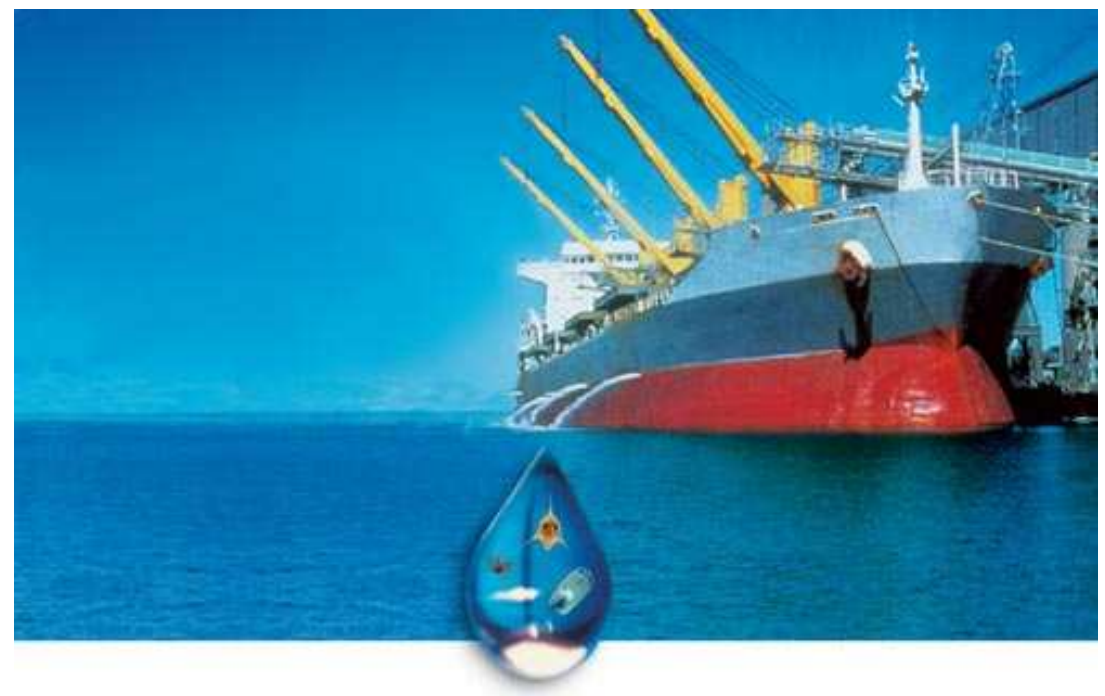

Figura 1.1: Navio descarregando água de lastro que possui organismos viáveis. Imagem meramente ilustrativa. Fonte: Mansfield (2012).

Recentemente o tratamento da água de lastro a bordo, envolvendo troca oceânica, fluxo contínuo, transbordamento, diluição etc têm sido empregado como tentativa para minimizar o problema [Pereira (2012)]. ${ }^{1}$

Para garantir a efetividade do tratamento, a IMO adotou [IMO (2008)] os seguintes limites de concentração de organismos vivos na água de lastro descarregada:

1. Menos que 10 organismos viáveis $/ \mathrm{m}^{3}$ com $50 \mu m$ ou mais em dimensão mínima (menor medida entre comprimento, largura e altura excluindo apêndices finos, como a antena sensorial e as cerdas). A maioria dos organismos nesta classe são zooplânctons.

2. Menos que 10 organismos viáveis $/ m l$ com dimensão mínima entre 10 e $50 \mu m$. A maioria dos organismos nesta classe são protozoários, incluindo zooplânctons.

3. Menos que as seguintes concentrações de indicadores de micróbios: (a) toxigênico Vibrio cholerae (sorotipos O1 e O139) < $1 \mathrm{cfu} / 100 \mathrm{ml}$; (b) Escherichia coli $<250 \mathrm{cfu} / 100 \mathrm{ml}$; (c) Enterococci intestinal $<100 \mathrm{cfu} / 100 \mathrm{ml}$.

Um dos problemas ainda em discussão na IMO é a regulamentação dos processos para avaliação do cumprimento dessas normas. Outro é a obtenção de amostras de água de lastro que forneçam estimativas com boas propriedades para a concentração de organismos viáveis ${ }^{2}$ do tanque de lastro [Carney et al. (2013)]. É impraticável avaliar toda a água de lastro descarregada por um navio, não só em função da quantidade (Tabela 1.1), quanto da velocidade com que é eliminada (Tabela 1.2)

\footnotetext{
${ }^{1}$ Neste texto o termo "água de lastro" será utilizado em lugar de "água de lastro tratada".

${ }^{2}$ Neste texto o termo "organismos" será utilizado em lugar de "organismos viáveis".
} 


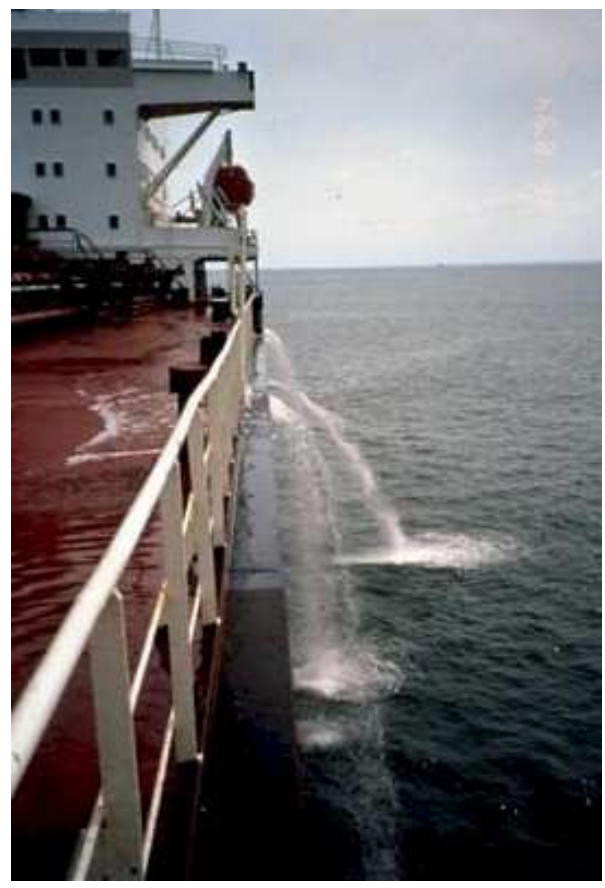

Figura 1.2: Navio descarregando água de lastro. Fonte: National Ballast Information Clearinghouse (2009).

e do tempo necessário para concretizar a análise em laboratório. Nesse contexto, é preciso recorrer à amostragem para avaliar se as normas são ou não satisfeitas. Basurko \& Mesbahi (2011), por exemplo, propuseram um esquema amostral baseado na distribuição Binomial, concluindo que é necessário amostrar entre $91 \%$ e 100\% da água de lastro de um navio para avaliar o cumprimento da primeira norma da IMO. Miller et al. (2011), por outro lado, utilizaram a distribuição Poisson para testar a hipótese de a água de lastro do navio estar dentro dos limites estabelecidos pela IMO. No primeiro caso, as frações amostrais são economicamente inviáveis. O segundo é baseado na suposição de que a concentração de organismos é uniforme no tanque, o que pode não ser realista, pois estudos mostram que organismos planctônicos são distribuídos de forma não-homogênea por todo o tanque de lastro [Murphy et al. (2002)].

Nosso objetivo é estudar esse problema, indicando alternativas que sejam mais realistas. No capítulo 2 descrevemos a proposta de Miller et al. (2011) e mostramos como pode ser adaptada para acomodar situações em que a concentração de organismos no tanque não é homogênea. Com essa finalidade, consideraremos amostragem estratificada sob a distribuição Poisson ou uma solução baseada em um processo de Poisson não-homogêneo. Também utilizamos a distribuição Binomial Negativa, obtida a partir de uma mistura Poisson/Gama, que além de acomodar a heterogeneidade e uma possível superdispersão ${ }^{3}$, permite avaliar a distribuição da concentração de organismos no tanque. No capítulo 3, consideraremos o mesmo problema discutido nas seções anteriores sob o

\footnotetext{
${ }^{3}$ Variância maior que a média.
} 
enfoque de estimação, indicando suas vantagens. Finalmente, no capítulo 4, apresentamos algumas conclusões, identificamos problemas de implementação prática e consideramos sugestões para futuras pesquisas.

Tabela 1.1: Volume de lastro nos tanques de alguns navios no porto de Tubarão/ES.

\begin{tabular}{lcc}
\hline \hline Navio & Comprimento $(m)$ & Lastro $($ ton $)$ \\
\hline Marina II & 190 & 12431 \\
Anita & 225 & 18905 \\
Barbara & 225 & 22200 \\
Alterego & 225 & 45439 \\
Aquagrace & 283 & 43600 \\
Cape Azalea & 289 & 59429 \\
Cape Provence & 289 & 52360 \\
Edward N & 314 & 59865 \\
Bergeland & 339 & 89234 \\
Alster N & 340 & 106985 \\
\hline \hline
\end{tabular}

Fonte: Pereira (2012)

Tabela 1.2: Taxa de bombeamento para alguns tipos de embarcação.

\begin{tabular}{lc}
\hline \hline Tipo de embarcação & Taxa de bombeamento $\left(\mathrm{m}^{3} / \mathrm{h}\right)$ \\
\hline Navio de granel seco & $5000-10000$ \\
Navio de minério & 10000 \\
Petroleiro & $10000-15000$ \\
Navio de containeres & $1000-2000$ \\
Balsa & $200-500$ \\
Navio de passageiros & $1000-2000$ \\
Pesqueiro & 50 \\
Pesqueiro industrial & 500 \\
Navio militar & $50-100$ \\
\hline \hline
\end{tabular}

Fonte: Pereira (2012) 


\section{Capítulo 2}

\section{Avaliação do cumprimento das}

\section{normas IMO por meio de testes de}

\section{hipóteses}

\subsection{Introdução}

Basurko \& Mesbahi (2011) utilizaram uma aproximação Normal para a distribuição Binomial com a finalidade de estimar a probabilidade de $1 \mathrm{~m}^{3}$ de água de lastro conter 10 ou mais organismos, chegando à conclusão de que é necessário amostrar entre $91 \%$ e 100\% do volume do tanque. Essas frações amostrais são obviamente inviáveis economicamente, dado que o volume de água de lastro em um navio pode chegar a milhares de $\mathrm{m}^{3}$. Uma alternativa para melhorar o plano amostral é considerar uma distribuição de probabilidade mais apropriada para modelar a distribuição de organismos no tanque.

\subsection{Amostragem sob a hipótese de homogeneidade da concentra- ção de organismos no tanque de lastro}

Para avaliar o cumprimento da norma IMO para organismos com dimensão mínima $\geq 50 \mu m^{1}$, Miller et al. (2011) utilizaram um método baseado na distribuição Poisson [Haight (1967)] para testar a hipótese de a concentração $(\lambda)$ de organismos no tanque de lastro ser $\leq 10 / \mathrm{m}^{3}$, ou seja,

\footnotetext{
${ }^{1} \mathrm{O}$ mesmo procedimento vale para organismos com dimensão entre 10 e $50 \mu \mathrm{m}$, pois nesse caso a norma IMO estabelece que a concentração $\lambda$ seja $\leq 10 \mathrm{org} / \mathrm{ml}$.
} 
para testar

$$
\left\{\begin{array}{c}
H_{0}: \lambda \leq 10 \\
\text { versus } \\
H_{1}: \lambda>10
\end{array}\right.
$$

Esses autores propõem duas alternativas de amostragem com essa finalidade. No primeiro caso considera-se uma amostra contendo uma única alíquota com volume $v \mathrm{~m}^{3}$ coletada do tanque de lastro, conta-se o número $X$ de organismos na amostra e supõe-se que esta observação segue uma distribuição Poisson com média $v \lambda$. Então, um procedimento de teste é

$$
\left\{\begin{array}{l}
\text { Rejeitar } H_{0}, \text { se } X \geq c \\
\text { ou } \\
\text { Nao rejeitar } H_{0}, \text { se } X<c,
\end{array}\right.
$$

em que $c$ é obtido a partir de

$$
\alpha=\mathbb{P}[X \geq c \mid \lambda=10] \quad \text { e } \quad \beta=\mathbb{P}[X<c \mid \lambda>10]
$$

com $\alpha$ denotando a probabilidade do erro do tipo I, $\beta$, a probabilidade do erro do tipo II e

$$
\mathbb{P}[X=x \mid \lambda]=e^{-v \lambda}(v \lambda)^{x} / x !, \quad \lambda>0, \quad x=0,1, \ldots
$$

Miller et al. (2011) apresentam alguns valores de $c$ para identificar o descumprimento da norma IMO com níveis de significância $\alpha=5 \%$ ou $10 \%$. Alguns desses valores estão reproduzidos na Tabela 2.1. Miller et al. (2011) também fixam volumes amostrais, tamanhos amostrais, níveis de significância e estudam as correspondentes curvas do poder $[1-\beta(\lambda)]$ do teste $(2.2)$, que reproduzimos na Figura 2.1.

Tabela 2.1: Limites de descumprimento da norma IMO $\left(\leq 10 \mathrm{org} / \mathrm{m}^{3}\right)$ usando a distribuição Poisson para níveis de significância $\alpha=0.05$ e $\alpha=0.10$ em termos do número de organismos (c) e da concentração correspondente $\left(\mathrm{org} / \mathrm{m}^{3}\right)$.

\begin{tabular}{ccccc}
\hline \hline Volume & \multicolumn{2}{c}{$\alpha=0.05$} & \multicolumn{2}{c}{$\alpha=0.10$} \\
\cline { 2 - 5 } amostral $(v)$ & $c$ & org $/ m^{3}$ & $c$ & org $/ m^{3}$ \\
\hline $1 m^{3}$ & 16 & 16.00 & 15 & 15.00 \\
$3 m^{3}$ & 40 & 13.34 & 38 & 12.67 \\
$5 m^{3}$ & 63 & 12.60 & 60 & 12.00 \\
$7 m^{3}$ & 85 & 12.14 & 82 & 11.71 \\
\hline \hline
\end{tabular}


Em muitas situações é necessário colher várias alíquotas de $v m^{3}$ que irão compor uma amostra. Neste caso, contam-se os números $\left(X_{1}, X_{2}, \ldots, X_{n}\right)$ de organismos nas alíquotas de $v m^{3}$ consideradas independentes. Supondo que cada uma dessas observações segue uma distribuição Poisson com média $v \lambda$, um procedimento de teste é

$$
\left\{\begin{array}{l}
\text { Rejeitar } H_{0}, \text { se } \sum_{i=1}^{n} X_{i} \geq c \\
\text { ou } \\
\text { Não rejeitar } H_{0}, \text { se } \sum_{i=1}^{n} X_{i}<c
\end{array}\right.
$$

em que $c$ é determinado a partir de

$$
\alpha=\mathbb{P}\left[\sum_{i=1}^{n} X_{i} \geq c \mid \lambda=10\right] \quad \text { e } \quad \beta=\mathbb{P}\left[\sum_{i=1}^{n} X_{i}<c \mid \lambda>10\right]
$$

com

$$
\mathbb{P}\left[\sum_{i=1}^{n} X_{i}=x \mid \lambda\right]=e^{-n v \lambda}(n v \lambda)^{x} / x !, \quad \lambda>0, \quad x=0,1, \ldots
$$
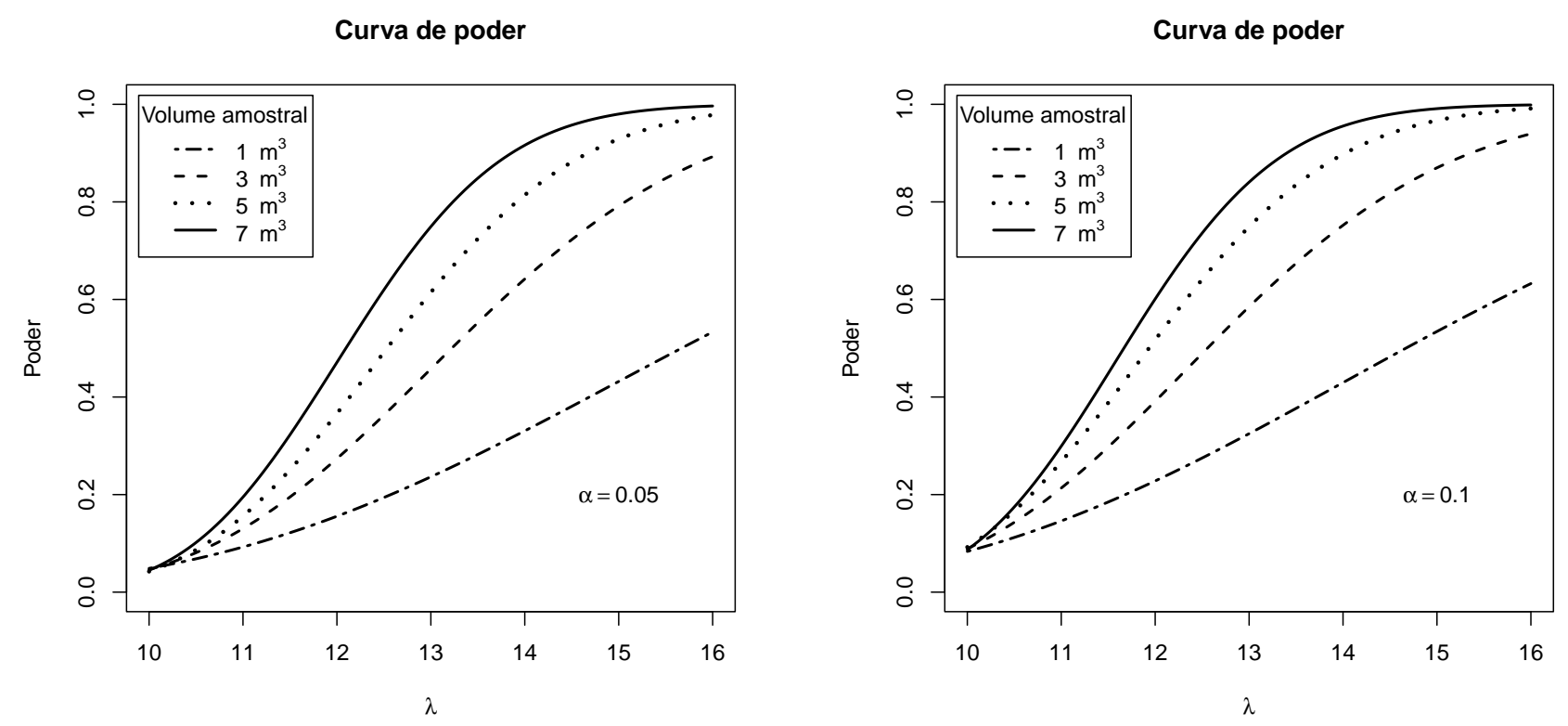

Figura 2.1: Curva de poder para o teste (2.2) com uma alíquota de $v m^{3}$ usando a distribuição Poisson.

Por meio de (2.5), pode-se calcular o menor tamanho amostral ou número de alíquotas de $v m^{3}$ (n) para o qual o teste (2.4) tenha poder $[1-\beta(\lambda)] \geq 1-b$ e nível de significância $(\alpha) \leq a$, com $a$ e $b$ fixados e diferentes valores para $\lambda$. Alguns valores estão apresentados na Tabela 2.2. Por exemplo, para detectar uma concentração $\lambda=14 \mathrm{org} / \mathrm{m}^{3}$ com nível de significância de no máximo 
5\% e poder no mínimo de $95 \%$ é necessário colher 10 alíquotas de $1 \mathrm{~m}^{3}$. Um pseudo-algoritmo para obtenção de $n$ está descrito no Apêndice D.

Tabela 2.2: Menor número ( $n$ ) de alíquotas de $v m^{3}$ para o qual o poder do teste (2.4) é $\geq 1-b$ e o nível de significância é $\leq a(a=0.05)$, usando a distribuição Poisson.

\begin{tabular}{ccccccccccccc}
\hline \hline & \multicolumn{4}{c}{$v=1 m^{3}$} & \multicolumn{4}{c}{$v=3 m^{3}$} \\
\cline { 2 - 13 } & \multicolumn{3}{c}{$b=0.05$} & \multicolumn{3}{c}{$b=0.10$} & \multicolumn{3}{c}{$b=0.05$} & \multicolumn{3}{c}{$b=0.10$} \\
\cline { 2 - 13 } & $c$ & $n$ & $1-\beta(\lambda)$ & $c$ & $n$ & $1-\beta(\lambda)$ & $c$ & $n$ & $1-\beta(\lambda)$ & $c$ & $n$ & $1-\beta(\lambda)$ \\
\hline 11 & 1207 & 116 & 0.951 & 961 & 92 & 0.900 & 1228 & 40 & 0.952 & 981 & 32 & 0.909 \\
12 & 340 & 32 & 0.956 & 277 & 26 & 0.914 & 361 & 12 & 0.964 & 298 & 10 & 0.931 \\
13 & 171 & 16 & 0.963 & 139 & 13 & 0.922 & 171 & 6 & 0.963 & 139 & 5 & 0.922 \\
14 & 107 & 10 & 0.962 & 85 & 8 & 0.916 & 107 & 4 & 0.962 & 107 & 4 & 0.962 \\
15 & 74 & 7 & 0.962 & 63 & 6 & 0.929 & 74 & 2 & 0.962 & 74 & 2 & 0.962 \\
\hline \hline
\end{tabular}

Usando os tamanhos amostrais apresentados na Tabela 2.2, foram simuladas contagens de organismos por meio de (2.3) considerando alíquotas amostrais de 1 e $3 \mathrm{~m}^{3}$, poder mínimo de 0.90 $(b=0.10)$, nível de significância de 0.05 e $\lambda=13 \mathrm{org} / \mathrm{m}^{3}$ ou $15 \mathrm{org} / \mathrm{m}^{3}$.

Para $\lambda=13$, o tamanho amostral para alíquotas de $1 \mathrm{~m}^{3}$ é 13 . Valores simulados para um tanque sob essas condições estão apresentados na Tabela 2.3. Considerando $\alpha=0.05$, temos $c=139$ e como $\sum_{i=1}^{n} X_{i}=149>c$, concluimos que o tanque não cumpre a norma IMO. Para $v=3 \mathrm{~m}^{3}$, o tamanho amostral necessário é 5 . As contagens simuladas foram 29, 27, 38, 41 e 42 o que implica que $\sum_{i=1}^{n} X_{i}=177$. Considerando $\alpha=0.05$ temos $c=139$ e como $\sum_{i=1}^{n} X_{i}>c$, concluimos que o tanque não cumpre a norma IMO.

Tabela 2.3: Contagens simuladas de organismos usando (2.6) com $n=13, \lambda=13$ e $v=1 \mathrm{~m}^{3}$.

\begin{tabular}{lllllllllllll}
\hline \hline \multicolumn{11}{c}{ Contagens $\left(\mathrm{org} / \mathrm{m}^{3}\right)$} \\
\hline 7 & 6 & 12 & 14 & 14 & 6 & 17 & 7 & 14 & 10 & 18 & 16 & 8 \\
\hline \hline
\end{tabular}

No caso $\lambda=15$, o tamanho amostral para alíquotas de $1 \mathrm{~m}^{3}$ é 6 . As contagens simuladas para um tanque sob essas condições foram $11,10,16,18,19$ e 13 o que implica que $\sum_{i=1}^{n} X_{i}=87$. Considerando $\alpha=0.05$ temos $c=63$ e como $\sum_{i=1}^{n} X_{i}>c$, concluimos que o tanque não cumpre a norma IMO. Para $v=3 \mathrm{~m}^{3}$, o tamanho amostral necessário é 2 . As contagens simuladas foram 38 e 37 o que implica que $\sum_{i=1}^{n} X_{i}=75$. Considerando $\alpha=0.05$ temos $c=74$ e como $\sum_{i=1}^{n} X_{i}>c$, concluimos que o tanque não cumpre a norma IMO. 


\subsection{Amostragem sob condições de heterogeneidade controlada da concentração de organismos no tanque de lastro}

A hipótese de homogeneidade da concentração de organismos no tanque de lastro admitida por Miller et al. (2011) não é realista. Essa concentração pode ser diferente em diferentes regiões do tanque, e este comportamento pode ser explicado pela ausência de luz, pelo formato do tanque, pelas mudanças na temperatura da água etc, [Murphy et al. (2002)]. Em alguns casos, a água de lastro é armazenada em vários tanques e em cada um deles a concentração de organismos pode ser diferente. Quando há informações sobre essa heterogeneidade, uma alternativa é aplicar amostragem estratificada, dividindo o tanque em $L$ regiões (estratos) com diferentes concentrações de organismos.

Suponha que no estrato $h(h=1,2, \ldots, L)$ a concentração seja $\lambda_{h}$ e o volume, $V_{h}$, o que implica que o volume total e concentração média de organismos no tanque são $V=\sum_{h=1}^{L} V_{h}$ e $\lambda=$ $V^{-1} \sum_{h=1}^{L} V_{h} \lambda_{h}$, respectivamente. Considere que em cada estrato $h$ seja observada uma alíquota com volume amostral $v_{h}=\left(V_{h} / V\right) v$ e suponha que o número de organismos nesse volume $\left(X_{h}\right)$ segue uma distribuição Poisson com média $v_{h} \lambda_{h}$. Então, $\sum_{h=1}^{L} X_{h}$ também segue uma distribuição Poisson, com média $v \lambda$. A hipótese a ser testada é (2.1), e o procedimento de teste é

$$
\left\{\begin{array}{l}
\text { Rejeitar } H_{0}, \text { se } \sum_{h=1}^{L} X_{h} \geq c \\
\text { ou } \\
\text { Não rejeitar } H_{0}, \text { se } \sum_{h=1}^{L} X_{h}<c,
\end{array}\right.
$$

em que o valor de $c$ é obtido de forma que

$$
\alpha=\mathbb{P}\left[\sum_{h=1}^{L} X_{h} \geq c \mid \lambda=10\right] \quad \text { e } \beta=\mathbb{P}\left[\sum_{h=1}^{L} X_{h}<c \mid \lambda>10\right]
$$

com

$$
\mathbb{P}\left[\sum_{h=1}^{L} X_{h}=x \mid \lambda\right]=e^{-v \lambda}(v \lambda)^{x} / x !, \quad \lambda>0, \quad x=0,1, \ldots
$$

Um pseudo-algoritmo para calcular o menor volume amostral total $(v)$ para qual o teste $(2.7)$ tem nível de significância $\leq a$ e poder $\geq 1-b$ está descrito no Apêndice D. Exemplos para diferentes valores de $a$ e $b$ estão indicados nas Tabelas 2.4 e 2.5 .

Considerando o volume amostral de cada estrato calculado nas Tabelas 2.4 e 2.5 foram simuladas contagens de organismos em cada estrato do tanque de lastro adotando nível de significância $\alpha=$ 
Tabela 2.4: Menores volumes amostrais $\left(v_{h}\right)$ para os quais o poder do teste (2.7) é $\geq 1-b(b=0.05) e$ $o$ nivel de significância é $\leq a(a=0.05)$, com $L=4$ estratos com volumes $V_{1}=1000 \mathrm{~m}^{3}, V_{2}=2000 \mathrm{~m}^{3}$, $V_{3}=500 \mathrm{~m}^{3}$ e $V_{4}=1500 \mathrm{~m}^{3}$, usando amostragem estratificada sob distribuição Poisson.

\begin{tabular}{|c|c|c|c|c|c|c|c|c|c|}
\hline \multicolumn{4}{|c|}{$\begin{array}{l}\text { Concentração por } \\
\text { estrato }\left(\mathrm{org} / m^{3}\right)\end{array}$} & \multirow{2}{*}{$\begin{array}{c}\text { Concentração } \\
\text { média }\left(\text { org } / m^{3}\right) \\
\lambda\end{array}$} & \multicolumn{4}{|c|}{$\begin{array}{l}\text { Volume amostral } \\
\text { por estrato }\left(\mathrm{m}^{3}\right)\end{array}$} & \multirow{2}{*}{-} \\
\hline$\lambda_{1}$ & $\lambda_{2}$ & $\lambda_{3}$ & $\lambda_{4}$ & & $v_{1}$ & $v_{2}$ & $v_{3}$ & $v_{4}$ & \\
\hline 9 & 10.5 & 9.5 & 12 & 10.6 & 73.51 & 147.02 & 36.75 & 110.26 & 3775 \\
\hline 11.5 & 11.5 & 10 & 12 & 11.5 & 10.34 & 20.67 & 5.17 & 15.5 & 554 \\
\hline 12.5 & 12 & 13 & 13.5 & 12.7 & 3.49 & 6.98 & 1.74 & 5.23 & 196 \\
\hline 12.5 & 14 & 14 & 13.5 & 13.6 & 2.01 & 4.03 & 1.01 & 3.02 & 117 \\
\hline 13 & 15.5 & 14 & 14.5 & 14.6 & 1.28 & 2.56 & 0.64 & 1.92 & 77 \\
\hline
\end{tabular}

Tabela 2.5: Menores volumes amostrais $\left(v_{h}\right)$ para os quais o poder do teste (2.7) é $\geq 1-b(b=0.10) e$ $o$ nivel de significância é $\leq a(a=0.05)$, com $L=4$ estratos com volumes $V_{1}=1000 \mathrm{~m}^{3}, V_{2}=2000 \mathrm{~m}^{3}$, $V_{3}=500 \mathrm{~m}^{3}$ e $V_{4}=1500 \mathrm{~m}^{3}$, usando amostragem estratificada sob distribuição Poisson.

\begin{tabular}{cccccccccc}
\hline \hline \multicolumn{3}{c}{$\begin{array}{c}\text { Concentração por } \\
\text { estrato }\left(\mathrm{org} / \mathrm{m}^{3}\right)\end{array}$} & $\begin{array}{c}\text { Concentração } \\
\text { média }\left(\mathrm{org} / \mathrm{m}^{3}\right)\end{array}$ & \multicolumn{4}{c}{$\begin{array}{c}\text { Volume amostral } \\
\text { por estrato }\left(\mathrm{m}^{3}\right)\end{array}$} & - \\
\hline$\lambda_{1}$ & $\lambda_{2}$ & $\lambda_{3}$ & $\lambda_{4}$ & $\lambda$ & $v_{1}$ & $v_{2}$ & $v_{3}$ & $v_{4}$ & $c$ \\
\hline 9 & 10.5 & 9.5 & 12 & 10.6 & 58.11 & 116.22 & 29.06 & 87.17 & 2994 \\
11.5 & 11.5 & 10 & 12 & 11.5 & 8.16 & 16.32 & 4.08 & 12.24 & 441 \\
12.5 & 12 & 13 & 13.5 & 12.7 & 2.74 & 5.48 & 1.37 & 4.11 & 156 \\
12.5 & 14 & 14 & 13.5 & 13.6 & 1.59 & 3.18 & 0.79 & 2.38 & 94 \\
13 & 15.5 & 14 & 14.5 & 14.6 & 1.01 & 2.02 & 0.50 & 1.51 & 62 \\
\hline \hline
\end{tabular}

0.05 , poder $1-\beta(\lambda)=0.90$ ou 0.95 , com os seguintes valores para $\lambda_{h}$.

(a) $\lambda_{1}=12.5, \lambda_{2}=12, \lambda_{3}=13, \lambda_{4}=13.5(\lambda=12.7)$.

Neste caso as contagens simuladas de organismos nos estratos foram 39, 69, 18 e 82, para um poder mínimo de 0.95, o que implica que $\sum_{h=1}^{L} X_{h}=208$. Considerando $\alpha=0.05$ temos que $c=196$ e como $\sum_{h=1}^{L} X_{h}>c$, concluimos que o tanque não cumpre a norma IMO. Para um poder mínimo de 0.90 as contagens simuladas nos estratos foram 30, 53, 13 e 66 o que implica que $\sum_{h=1}^{L} X_{h}=162$. Considerando $\alpha=0.05$ temos que $c=156$ e como $\sum_{h=1}^{L} X_{h}>c$, concluimos que o tanque não cumpre a norma IMO.

(b) $\lambda_{1}=13, \lambda_{2}=15.5, \lambda_{3}=14, \lambda_{4}=14.5(\lambda=14.6)$.

Neste caso as contagens simuladas de organismos nos estratos foram 14, 29, 6 e 35, para um poder mínimo de 0.95 , o que implica que $\sum_{h=1}^{L} X_{h}=84$. Considerando $\alpha=0.05$ temos que $c=77$ e como $\sum_{h=1}^{L} X_{h}>c$, concluimos que o tanque não cumpre a norma IMO. Para um poder mínimo de 0.90 as contagens simuladas nos estratos foram $11,22,5$ e 28 o que implica que $\sum_{h=1}^{L} X_{h}=66$. Considerando $\alpha=0.05$ temos que $c=62$ e como $\sum_{h=1}^{L} X_{h}>c$, concluimos que o tanque não cumpre a norma IMO.

Suponha agora que a concentração de organismos varie continuamente ao longo de uma dimen- 
são, como o volume de água deslastrado, $v$, durante um processo que irá descarregar um volume total $V$ de água de lastro. Podemos então utilizar um processo de Poisson não-homogêneo para modelar a distribuição do número de organismos $\{X(v)\}$ no volume de água $v$ deslastrado do tanque em que a concentração é $\lambda(v)$ [Ver Ross (1996) ou Basawa \& Rao (1980) para detalhes]. O número de organismos observados durante o processo de deslastro que descarrega continuamente de 0 até $V$ unidades de volume de água segue uma distribuição Poisson com média $\int_{0}^{V} \lambda(v) d v$. A hipótese a ser testada é (2.1), em que $\lambda=V^{-1} \int_{0}^{V} \lambda(v) d v$, corresponde à concentração média de organismos no tanque. A forma da função $\lambda(v)$ determina a variação da concentração de organismos no tanque com o volume de água deslastrado. Alguns exemplos empregados na prática são

$$
\lambda(v)=\eta \theta v^{\theta-1}, \quad \lambda(v)=\eta \theta \exp (-\theta v) \quad \text { ou } \quad \lambda(v)=\eta \theta /(1+\theta v) .
$$

Suponha que o processo $\{X(v)\}$ seja observado em $n$ instantes do processo de deslastro $\left(v_{i}, i=\right.$ $1,2, \ldots, n)$, ou entre dois instantes com diferença desprezível em relação ao volume $V$. Neste caso, a verossimilhança é

$$
L(\eta, \theta)=\prod_{i=1}^{n} \frac{e^{-\lambda\left(v_{i}\right)} \lambda\left(v_{i}\right)^{x_{i}}}{x_{i} !}, \quad x_{i}=0,1, \ldots
$$

em que $x_{i}$ é o número de organismos observados no volume $v_{i}$ de água deslastrado. Estimativas de máxima verossimilhança para $\eta$ e $\theta$, são obtidas maximizando (2.9), e utilizadas para calcular a estimativa de máxima verossimilhança de $\lambda$ por meio do princípio da invariância [Roussas (1997)]. A matriz de covariância de $\eta$ e $\theta$ é calculada através da matriz de informação de Fisher [Ver Sen et al. (2009), por exemplo]. O erro padrão da estimativa de máxima verossimilhança de $\lambda$ é calculado por meio do método Delta [Ver Sen et al. (2009), por exemplo]. O procedimento de teste para a hipótese (2.1) é

$$
\left\{\begin{array}{l}
\text { Rejeitar } H_{0}, \text { se } \xi_{W} \geq c \\
\text { ou } \\
\text { Não rejeitar } H_{0}, \text { se } \xi_{W}<c,
\end{array}\right.
$$

em que $\xi_{W}=(\widehat{\lambda}-\lambda) / \hat{\sigma}_{\hat{\lambda}}, \widehat{\lambda}$ é a estimativa de máxima verossimilhança de $\lambda$ e $\widehat{\sigma}_{\hat{\lambda}}$ é a estimativa do erro padrão de $\hat{\lambda}$. Fixando um nível de significância $\alpha$, o valor de $c$ é obtido de tal forma que

$$
\alpha=\mathbb{P}[Z \geq c \mid \lambda=10],
$$


em que $Z$ tem distribuição Normal padrão. Utilizamos a função mle do pacote stats 4 do software R [R Development Core Team (2012)], baseada no método BFGS de otmização [Broyden (1970); Fletcher (1970); Goldfarb (1970); Shanno (1970)], para calcular as estimativas de máxima verossimilhança de $\eta$ e $\theta$ com base nas observações da quantidade de organismos $\left(x_{i}\right)$ em cada volume de água deslastrado $v_{i}$. Os códigos podem ser obtidos em www.ime.usp.br/〜 eliardo.

Em seguida apresentamos detalhes para dois casos particulares.

\section{Função potência:}

$$
\lambda(v)=\eta \theta v^{\theta-1}, \quad \eta, \theta>0 .
$$

Neste caso o comportamento da concentração de organismos de acordo com o volume de água deslastrado pode assumir várias formas dependendo do valor do parâmetro $\theta$ (Figura 2.2). As estimativas de $\eta$ e $\theta$ são obtidas por meio da solução do sistema de equações.

$$
\left\{\begin{array}{l}
-\widehat{\theta} \sum_{i=1}^{n} v_{i}+(\widehat{\theta} / \widehat{\eta}) \sum_{i=1}^{n} x_{i}=0, \\
-\widehat{\eta} \sum_{i=1}^{n} v_{i}^{\widehat{\theta}-1}-\widehat{\eta} \widehat{\theta} \sum_{i=1}^{n} v_{i}^{\widehat{\theta}-1} \log v_{i}+(\widehat{\eta} / \widehat{\theta}) \sum_{i=1}^{n} x_{i}+\sum_{i=1}^{n} x_{i} \log v_{i}=0 .
\end{array}\right.
$$

Suponha que em um processo de deslastro seja descarregado um volume total de água de $V=$ $5 \mathrm{~m}^{3}$ e que sejam coletadas 9 observações em diferentes instantes do processo. Consideramos as seguintes situações.

(a) $\theta<1$

Neste caso a concentração de organismos diminui à medida que o volume de água deslastrado aumenta. Fixamos valores $\eta=20$ e $\theta=0.7$, o que implica $\lambda=\eta V^{\theta-1}=12.341$. Contagens simuladas sob estas condições estão apresentadas na Tabela 2.6 e na Figura 2.3. Na Tabela 2.7 encontram-se as estimativas de máxima verossimilhança obtidas a partir das contagens simuladas. O valor da estatística de Wald é $\widehat{\xi}_{W}=1.483$. Considerando $\alpha=0.05$ temos $c=1.645$ e como $\widehat{\xi}_{W}<c$, concluimos que o tanque cumpre a norma IMO.

Tabela 2.6: Contages simuladas de organismos usando (2.9) $\operatorname{com} \lambda(v)$ dado pela função potência (2.10) $e$ $\theta<1 \quad(\eta=20$ e $\theta=0.7)$.

\begin{tabular}{cccccccccc}
\hline \hline \multicolumn{10}{c}{ Contagens $\left(\mathrm{org} / \mathrm{m}^{3}\right)$} \\
\hline$v_{i}\left(m^{3}\right)$ & 0.1 & 0.7 & 1.3 & 1.9 & 2.5 & 3.1 & 3.7 & 4.3 & 4.9 \\
$x_{i}\left(v_{i}\right)$ & 27 & 13 & 7 & 8 & 15 & 9 & 12 & 10 & 8 \\
\hline \hline
\end{tabular}




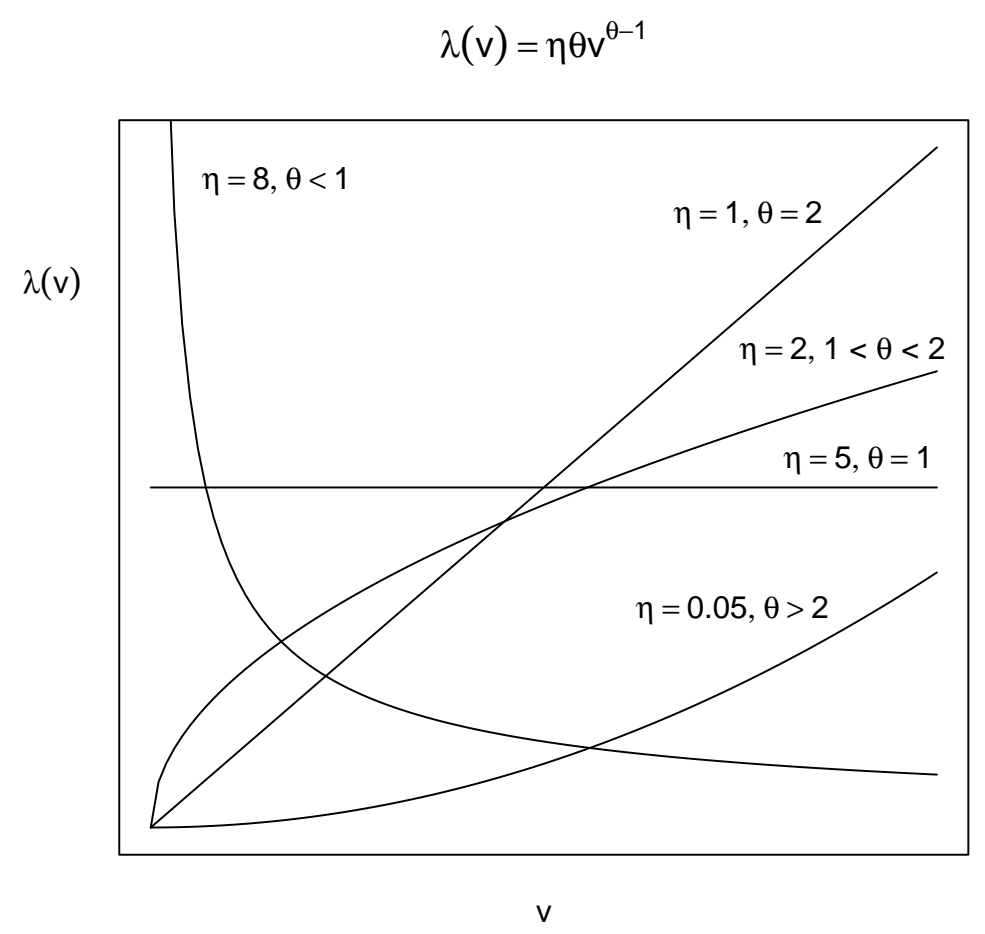

Figura 2.2: Variação da concentração de organismos ao longo do volume (v) de água deslastrado do tanque de acordo com uma função potência (2.10). Obs: Para cada valor de $\theta$, a forma da curva é similar para outros valores de $\eta$.

Tabela 2.7: Estimativas para o modelo (2.9) usando a função potência (2.10) para $\eta=20, \theta=0.7$ $(\lambda=12.341)$.

\begin{tabular}{cccc}
\hline \hline Parâmetro & Estimativa & Erro padrão & IC $(95 \%)$ \\
\hline$\eta$ & 18.113 & 2.486 & {$[13.754 ; 23.590]$} \\
$\theta$ & 0.727 & 0.068 & {$[0.596 ; 0.864]$} \\
$\lambda$ & 11.67 & 1.126 & - \\
\hline \hline
\end{tabular}

(b) $\theta=1$.

Quando $\theta=1$, a concentração de organismos é constante ao longo do processo de deslastro da água do tanque. Fixamos valores $\eta=15$ e $\theta=1$, o que implica $\lambda=\eta V^{\theta-1}=15$. Contagens simuladas sob estas condições estão apresentadas na Tabela 2.8 e na Figura 2.4. Na Tabela 2.9 encontram-se as estimativas de máxima verossimilhança usando as contagens simuladas. O valor da estatística de Wald é $\widehat{\xi}_{W}=3.235$. Considerando $\alpha=0.05$ temos $c=1.645$ e como $\widehat{\xi}_{W}>c$, concluimos que o tanque não cumpre a norma IMO.

(c) $1<\theta<2$.

Neste caso, a concentração de organismos aumenta ao longo do processo de deslastro da água do tanque de forma que a concavidade da curva correspondente é voltada para 


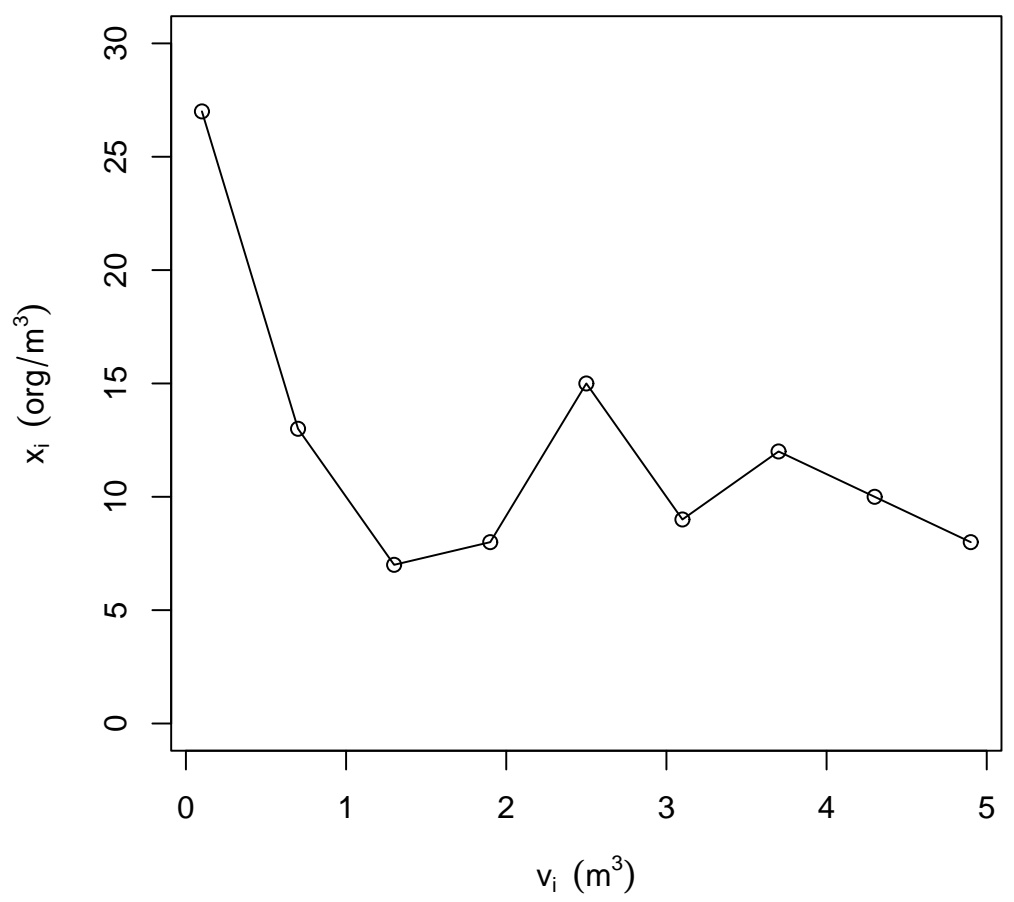

Figura 2.3: Simulação da contagem de organismos para diferentes volumes $\left(v_{i}\right)$ de água deslastrada do tanque usando (2.9) com $\lambda(v)$ dado pela função potência (2.10) e $\theta<1 \quad(\eta=20$ e $\theta=0.7)$.

Tabela 2.8: Contagens simuladas de organismos usando (2.9) $\operatorname{com} \lambda(v)$ dado pela função potência (2.10) $\operatorname{com} \theta=1(\eta=15$ e $\theta=1)$.

\begin{tabular}{cccccccccc}
\hline \hline \multicolumn{10}{c}{ Contagens $\left(\mathrm{org} / \mathrm{m}^{3}\right)$} \\
\hline$v_{i}\left(m^{3}\right)$ & 0.1 & 0.7 & 1.3 & 1.9 & 2.5 & 3.1 & 3.7 & 4.3 & 4.9 \\
$x_{i}\left(v_{i}\right)$ & 14 & 12 & 9 & 11 & 20 & 13 & 17 & 16 & 14 \\
\hline \hline
\end{tabular}

Tabela 2.9: Estimativas para o modelo (2.9) usando a função potência (2.10) para $\eta=15, \theta=1(\lambda=15)$.

\begin{tabular}{cccc}
\hline \hline Parâmetro & Estimativa & Erro padrão & IC $(95 \%)$ \\
\hline$\eta$ & 12.888 & 1.968 & {$[9.439 ; 17.196]$} \\
$\theta$ & 1.054 & 0.081 & {$[0.903 ; 1.220]$} \\
$\lambda$ & 14.063 & 1.256 & - \\
\hline \hline
\end{tabular}

baixo. Fixamos valores $\eta=10$ e $\theta=1.5$, o que implica $\lambda=\eta V^{\theta-1}=22.361$. Contagens simuladas sob estas condições estão apresentadas na Tabela 2.10 e na Figura 2.5. Na Tabela 2.11 encontram-se as estimativas de máxima verossimilhança usando as contagens simuladas. O valor da estatística de Wald é $\widehat{\xi}_{W}=7.378$. Considerando $\alpha=0.05$ temos $c=1.645$ e como $\widehat{\xi}_{W}>c$, concluimos que o tanque não cumpre a norma IMO.

(d) $\theta=2$.

Quando $\theta=2$, a concentração de organismos aumenta de forma linear ao longo do pro- 


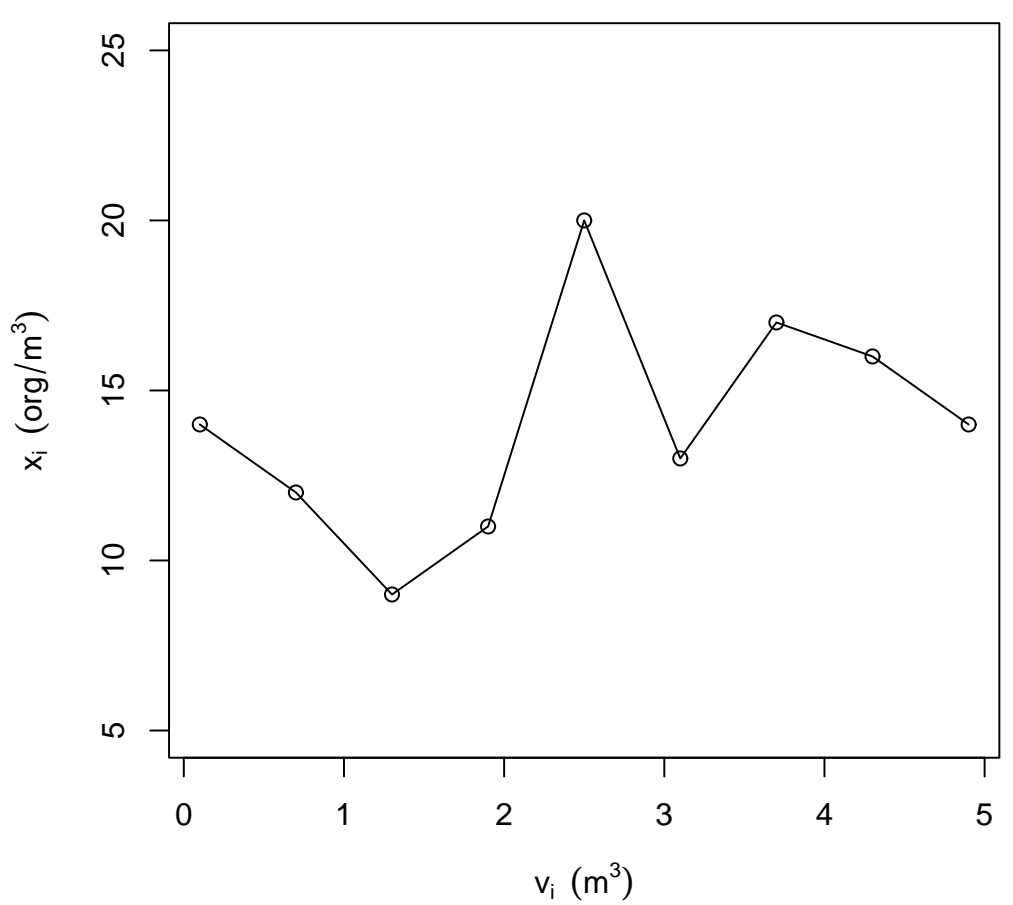

Figura 2.4: Simulação da contagem de organismos para diferentes volumes $\left(v_{i}\right)$ de água deslastrada do tanque usando (2.9) $\operatorname{com} \lambda(v)$ dado pela função potência (2.10) $\operatorname{com} \theta=1 \quad(\eta=15$ e $\theta=1)$.

Tabela 2.10: Contagens simuladas de organismos usando (2.9) com $\lambda(v)$ dado pela função potência (2.10) $e 1<\theta<2(\eta=10$ e $\theta=1.5)$.

\begin{tabular}{cccccccccc}
\hline \hline \multicolumn{11}{c}{ Contagens $\left(\mathrm{org} / \mathrm{m}^{3}\right)$} \\
\hline$v_{i}\left(m^{3}\right)$ & 0.1 & 0.7 & 1.3 & 1.9 & 2.5 & 3.1 & 3.7 & 4.3 & 4.9 \\
$x_{i}\left(v_{i}\right)$ & 4 & 10 & 10 & 16 & 30 & 24 & 32 & 33 & 32 \\
\hline \hline
\end{tabular}

Tabela 2.11: Estimativas para o modelo (2.9) usando a função potência (2.10) para $\eta=10, \theta=1.5$ $(\lambda=22.361)$.

\begin{tabular}{cccc}
\hline \hline Parâmetro & Estimativa & Erro padrão & IC $(95 \%)$ \\
\hline$\eta$ & 7.689 & 1.490 & {$[5.159 ; 11.036]$} \\
$\theta$ & 1.638 & 0.110 & {$[1.434 ; 1.866]$} \\
$\lambda$ & 21.471 & 1.555 & - \\
\hline \hline
\end{tabular}

cesso de deslastro da água do tanque. Fixamos valores $\eta=1.5$ e $\theta=2$, o que implica $\lambda=\eta V^{\theta-1}=7.5$. Contagens simuladas sob estas condições estão apresentadas na Tabela 2.12 e na Figura 2.6. Na Tabela 2.13 encontram-se as estimativas de máxima verossimilhança utilizando as contagens simuladas. O valor da estatística de Wald é $\widehat{\xi}_{W}=-2.955$. Considerando $\alpha=0.05$ temos $c=1.645$ e como $\widehat{\xi}_{W}<c$, concluimos que o tanque cumpre com a norma IMO. 


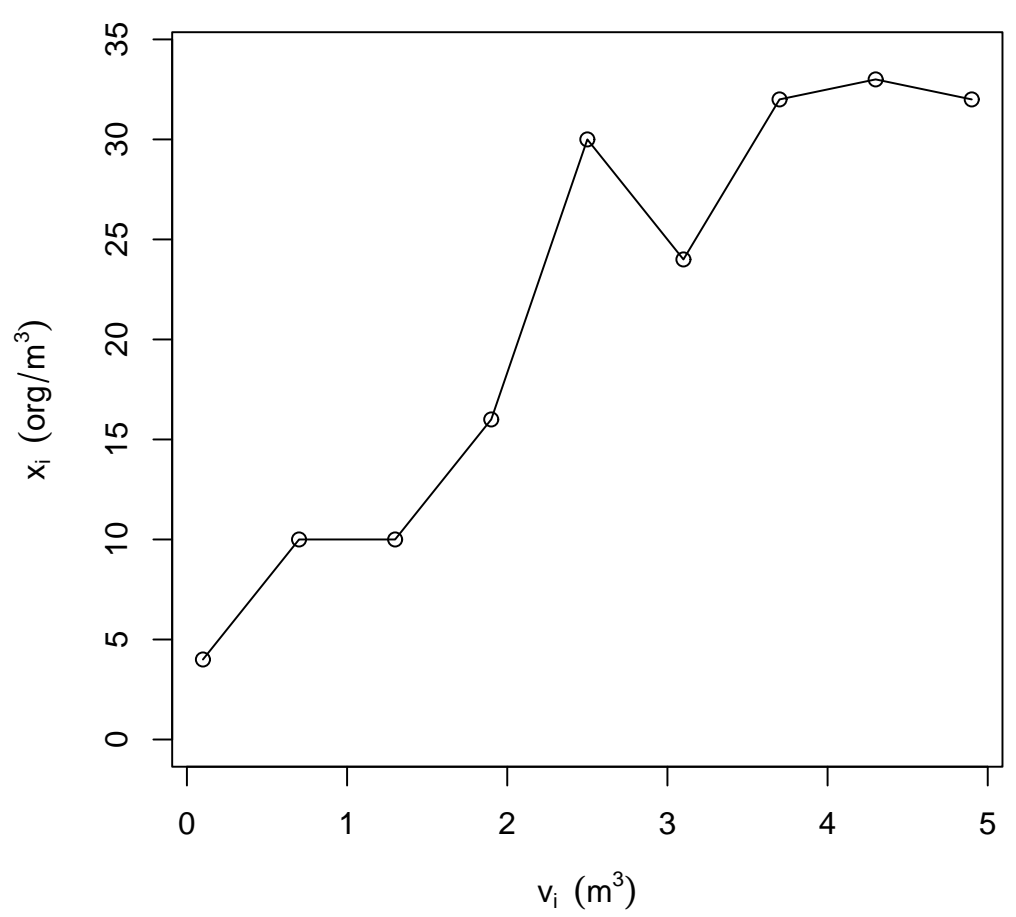

Figura 2.5: Simulação da contagem de organismos para diferentes volumes $\left(v_{i}\right)$ de água deslastrada do tanque usando (2.9) com $\lambda(v)$ dado pela função potência (2.10) e $1<\theta<2$ ( $\eta=10$ e $\theta=1.5)$.

Tabela 2.12: Contagens simuladas de organismos usando $2.9 \mathrm{com} \lambda(v)$ dada pela função potência (2.10) para $\theta=2(\eta=1.5$ e $\theta=2)$.

\begin{tabular}{cccccccccc}
\hline \hline \multicolumn{10}{c}{ Contagens $\left(\mathrm{org} / \mathrm{m}^{3}\right)$} \\
\hline$v_{i}\left(m^{3}\right)$ & 0.1 & 0.7 & 1.3 & 1.9 & 2.5 & 3.1 & 3.7 & 4.3 & 4.9 \\
$x_{i}\left(v_{i}\right)$ & 0 & 1 & 1 & 4 & 12 & 8 & 13 & 14 & 14 \\
\hline \hline
\end{tabular}

Tabela 2.13: Estimativas para o modelo (2.9) usando a função potência (2.10) para $\eta=1.5, \theta=2(\lambda=7.5$ ).

\begin{tabular}{cccc}
\hline \hline Parâmetro & Estimativa & Erro padrão & IC (95\%) \\
\hline$\eta$ & 0.798 & 0.395 & {$[0.281 ; 1.948]$} \\
$\theta$ & 2.379 & 0.291 & {$[1.940 ; 2.999]$} \\
$\lambda$ & 7.340 & 0.900 & - \\
\hline \hline
\end{tabular}

(e) $\theta>2$.

Neste caso, a concentração de organismos aumenta com o volume de água deslastrado do tanque, de forma que a concavidade da curva correspondente é voltada para cima. Fixamos $\eta=2$ e $\theta=3$, o que implica $\lambda=\eta V^{\theta-1}=50$. Contagens simuladas sob estas condições estão apresentadas na Tabela 2.14 e na Figura 2.7. Na Tabela 2.15 encontramse as estimativas de máxima verossimilhança usando as contagens simuladas. $\mathrm{O}$ valor da estatística de Wald é $\widehat{\xi}_{W}=17.176$. Considerando $\alpha=0.05$ temos $c=1.645$ e como 


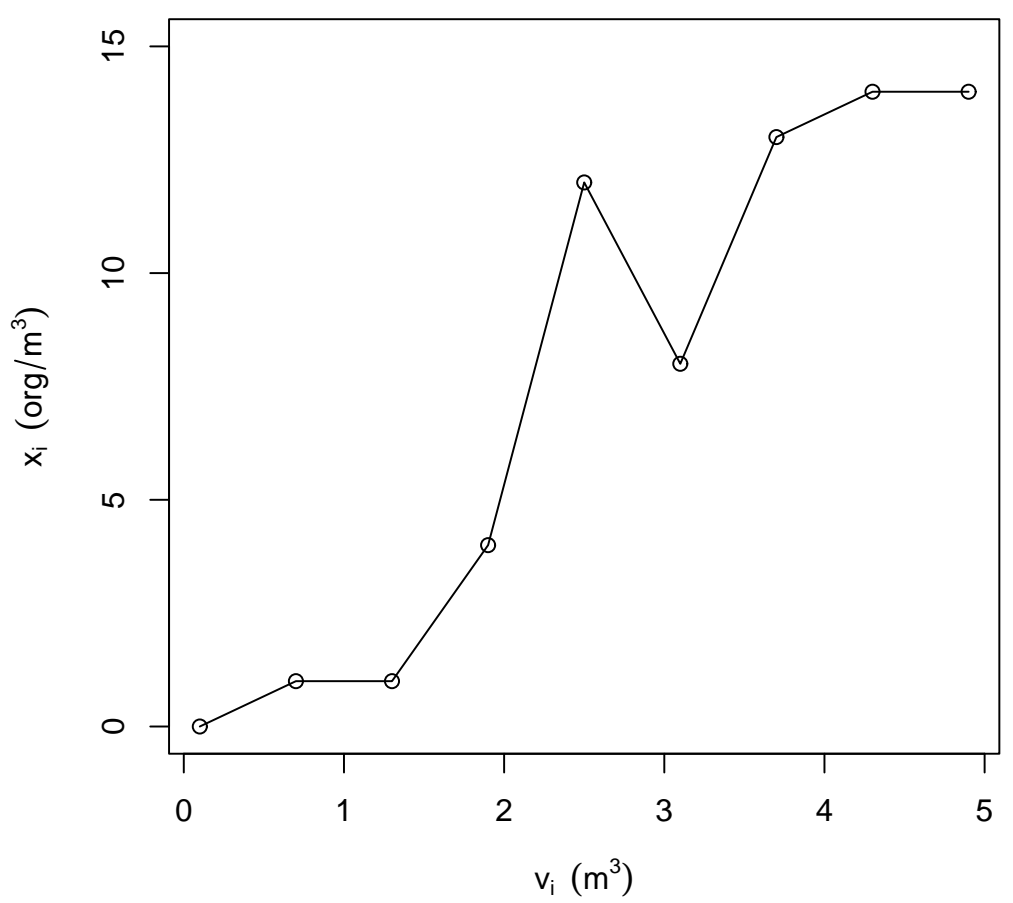

Figura 2.6: Simulação da contagem de organismos para diferentes volumes $\left(v_{i}\right)$ de água deslastrada do tanque usando $2.9 \operatorname{com} \lambda(v)$ dada pela função potência (2.10) para $\theta=2(\eta=1.5$ e $\theta=2)$.

$$
\widehat{\xi}_{W}>c \text {, concluimos que o tanque não cumpre a norma IMO. }
$$

Tabela 2.14: Contagens simuladas de organismos usando (2.9) com $\lambda(v)$ dada pela função potência (2.10) para $\theta>2(\eta=2$ e $\theta=3)$.

\begin{tabular}{cccccccccc}
\hline \hline \multicolumn{10}{c}{ Contagens $\left(\mathrm{org} / \mathrm{m}^{3}\right)$} \\
\hline$v_{i}\left(m^{3}\right)$ & 0.1 & 0.7 & 1.3 & 1.9 & 2.5 & 3.1 & 3.7 & 4.3 & 4.9 \\
$x_{i}\left(v_{i}\right)$ & 0 & 2 & 5 & 17 & 46 & 54 & 88 & 114 & 142 \\
\hline \hline
\end{tabular}

Tabela 2.15: Estimativas para o modelo (2.9) usando a função potência (2.10) para $\eta=2, \theta=3(\lambda=50)$.

\begin{tabular}{cccc}
\hline \hline Parâmetro & Estimativa & Erro padrão & IC (95\%) \\
\hline$\eta$ & 1.557 & 0.383 & {$[0.948 ; 2.489]$} \\
$\theta$ & 3.153 & 0.147 & {$[2.874 ; 3.450]$} \\
$\lambda$ & 49.817 & 2.318 & - \\
\hline \hline
\end{tabular}

\section{Função recíproca:}

$$
\lambda(v)=\eta \theta /(1+\theta v), \quad \eta, \theta>0 .
$$

Neste caso a concentração de organismos decresce à medida que o volume de água deslastrada do tanque aumenta sendo que o valor da concentração no início do processo $(v=0)$ é $\eta \theta$. A Figura 2.8 ilustra o comportamento desta função. Fixamos valores $\eta=23$ e $\theta=$ 


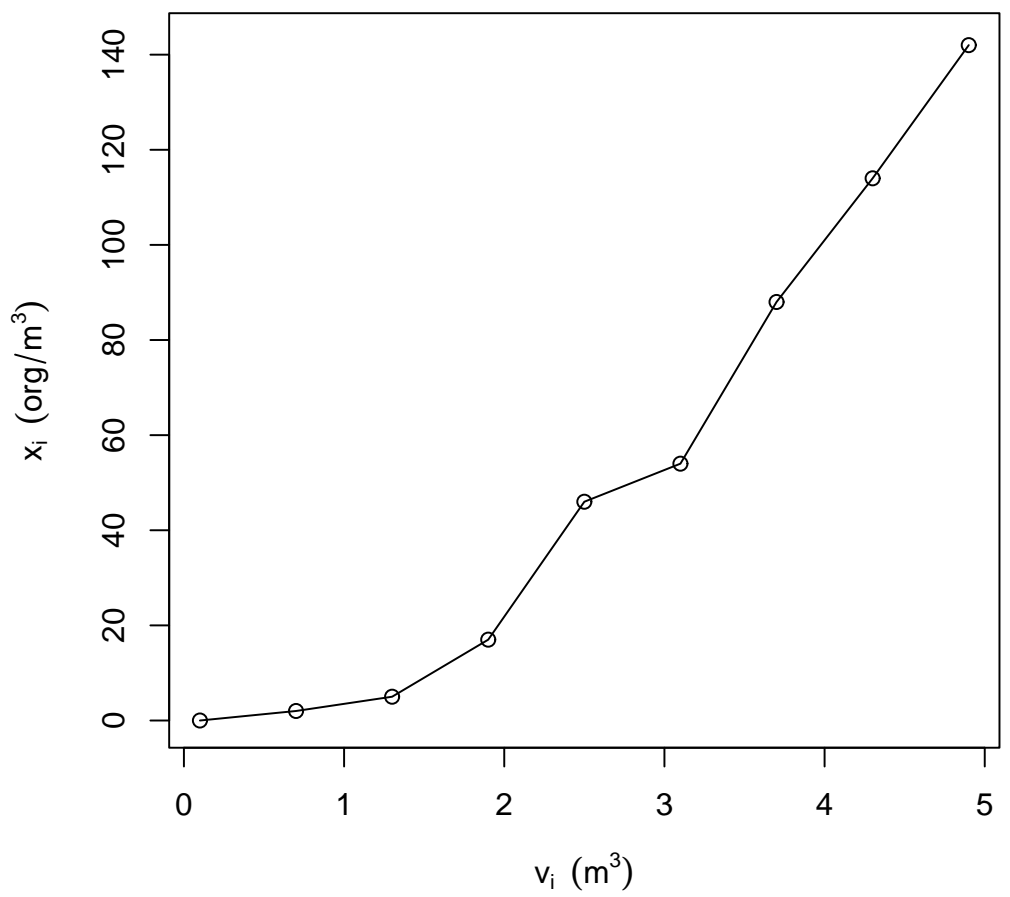

Figura 2.7: Simulação da contagem de organismos para diferentes volumes $\left(v_{i}\right)$ de água deslastrada do tanque usando (2.9) $\operatorname{com} \lambda(v)$ dada pelaa função potência (2.10) para $\theta>2(\eta=2$ e $\theta=3)$.

5, o que implica $\lambda=(\eta / V) \log (1+\theta V)=14.987$. E seguindo a mesma configuração da simulação para a função (2.10) simulamos as contagens de organismos que estão apresentadas na Tabela 2.16 e na Figura 2.9. As estimativas de máxima verossimilhança de $\eta$ e $\theta$ são obtidas através do sistema de equações (2.12). Na Tabela 2.17 encontram-se as estimativas de máxima verossimilhança de $\eta, \theta$ e $\lambda$. O valor da estatística de Wald é $\widehat{\xi}_{W}=3.301$. Considerando $\alpha=0.05$ temos $c=1.645$ e como $\widehat{\xi}_{W}>c$, concluimos que o tanque não cumpre a norma IMO.

$$
\left\{\begin{array}{l}
-\widehat{\theta} \sum_{i=1}^{n} \frac{1}{1+\widehat{\theta} v_{i}}+(\widehat{\theta} / \widehat{\eta}) \sum_{i=1}^{n} x_{i}=0 \\
-\widehat{\eta} \sum_{i=1}^{n}\left(1+\widehat{\theta} v_{i}\right)^{-2}+\widehat{\eta} \widehat{\theta} \sum_{i=1}^{n} x_{i}-\sum_{i=1}^{n} \frac{x_{i} v_{i}}{1+\hat{\theta} v_{i}}=0
\end{array}\right.
$$

Tabela 2.16: Contagens simuladas de organismos para o modelo (2.9) usando a função recíproca (2.11) para $\eta=23, \theta=5(\lambda=14.987)$.

\begin{tabular}{cccccccccc}
\hline \hline \multicolumn{110}{c}{ Contagens $\left(\mathrm{org} / \mathrm{m}^{3}\right)$} \\
\hline$v_{i}\left(m^{3}\right)$ & 0.1 & 0.7 & 1.3 & 1.9 & 2.5 & 3.1 & 3.7 & 4.3 & 4.9 \\
$x_{i}\left(v_{i}\right)$ & 75 & 22 & 9 & 7 & 13 & 6 & 8 & 6 & 4 \\
\hline \hline
\end{tabular}




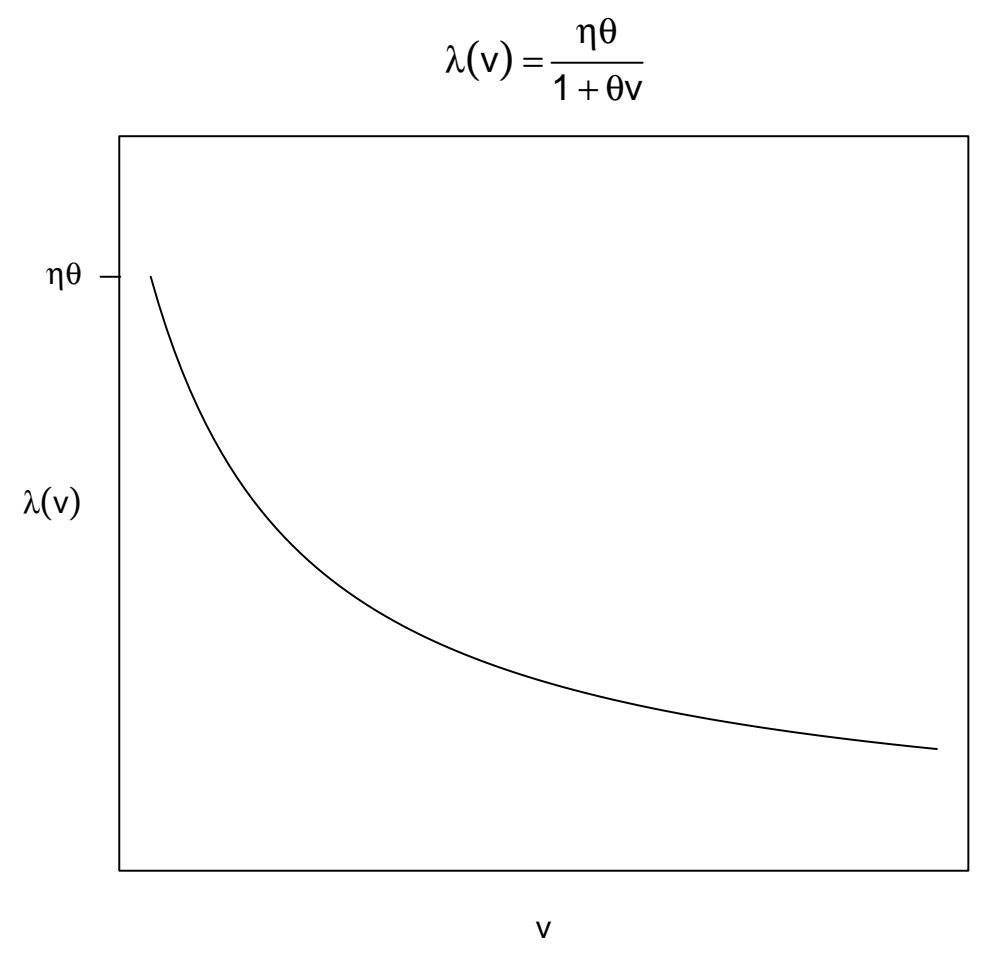

Figura 2.8: Variação da concentração de organismos no tanque $(\lambda(v))$ de lastro ao longo do volume de água (v) deslastrado do tanque com comportamento de uma função recíproca (2.11).

Tabela 2.17: Estimativas para o modelo (2.9) usando a função recíproca (2.11) para $\eta=23, \theta=5$ $(\lambda=14.987)$.

\begin{tabular}{cccc}
\hline \hline Parâmetro & Estimativa & Erro padrão & IC (95\%) \\
\hline$\eta$ & 20.909 & 2.984 & {$[15.759 ; 27.632]$} \\
$\theta$ & 5.462 & 1.689 & {$[3.080 ; 10.741]$} \\
$\lambda$ & 13.981 & 1.206 & - \\
\hline \hline
\end{tabular}

\subsection{Amostragem sob heterogeneidade aleatória da concentração de organismos no tanque de lastro}

Quando não há informações a priori sobre a distribuição da concentração dos organismos no tanque, uma alternativa é considerá-la como uma variável aleatória $\mathcal{L}$ com distribuição Gama, i.e., para o qual a função densidade é

$$
f(\ell)=\frac{1}{\Gamma(k)}\left(\frac{\ell k}{\lambda}\right)^{k} \exp (-k \ell / \lambda) / \ell, \quad \ell>0
$$

Neste contexto, a distribuição da concentração de organismos no tanque pode assumir várias 


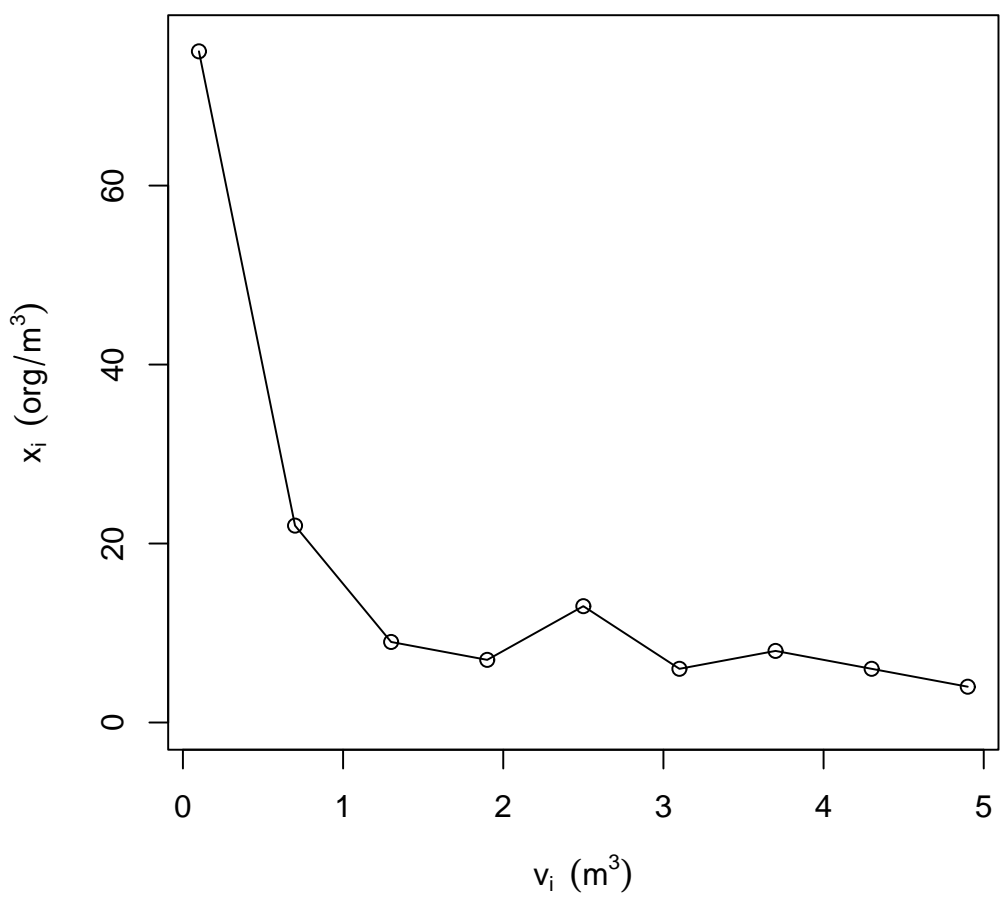

Figura 2.9: Simulação da contagem de organismos para diferentes volumes $\left(v_{i}\right)$ de água deslastrada do tanque usando (2.9) com $\lambda(v)$ dada pela função recíproca (2.11) $(\eta=23$ e $\theta=5)$.

formas:

- Muitas regiões no tanque de lastro com concentração baixa e poucas com concentração alta $(\lambda=3, k=1$, por exemplo).

- Muitas regiões do tanque de lastro com concentrações diferentes $(\lambda=18, k=3$, por exemplo).

- Muitas regiões do tanque de lastro com concentrações em torno de um determinado valor $(\lambda=5, k=20$ ou $\lambda=20, k=350$, por exemplo).

Gráficos correspondentes a esses três casos estão apresentados na Figura 2.10. Usando (2.13) pode-se mostrar que $\mathbb{E}[\mathcal{L}]=\lambda$ e $\operatorname{Var}[\mathcal{L}]=\lambda^{2} / k$. Condicionalmente a $\ell$ e $k$, suponha que a contagem de organismos em uma alíquota de $1 \mathrm{~m}^{3}, X \mid \ell$, segue uma distribuição Poisson com média $\ell$. Isso implica que $X$ segue uma distribuição Binomial Negativa com média $\lambda$ e parâmetro de dispersão $k$, i.e., com função de probabilidade

$$
\mathbb{P}[X=x \mid \lambda, k]=\frac{\Gamma(k+x)}{\Gamma(x+1) \Gamma(k)}\left(\frac{\lambda}{\lambda+k}\right)^{x}\left(\frac{k}{\lambda+k}\right)^{k}, \quad \lambda, k>0, \quad x=0,1,2, \ldots,
$$

em que $\Gamma(x)=\int_{0}^{\infty} t^{x-1} e^{-t} d t$ é a função gama. Neste contexto, $\mathbb{E}[X]=\lambda$ e $\operatorname{Var}[X]=\lambda+\lambda^{2} / k$, 


\section{Distribuição da concentração de organismos no tanque}

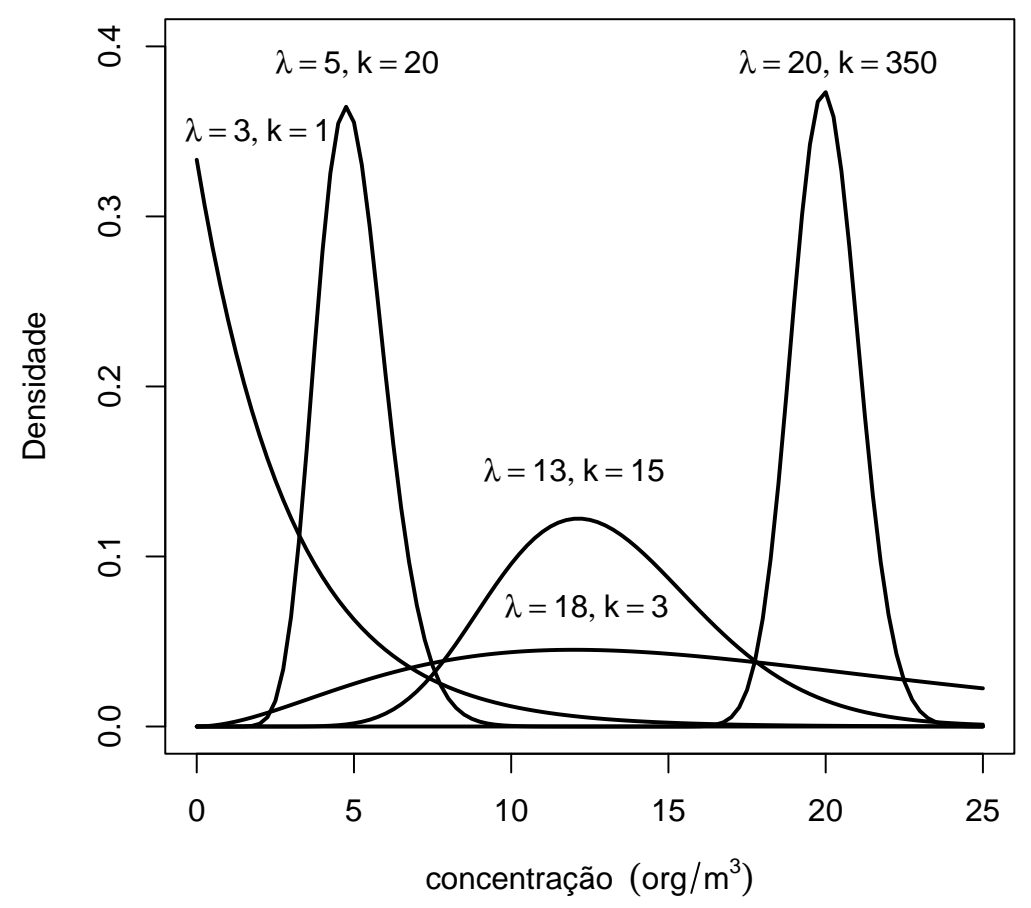

Figura 2.10: Algumas formas para a distribuição da concentração de organismos no tanque de lastro.

indicando que essa distribuição pode acomodar uma possível superdispersão, i.e., variância maior que a média. Esta distribuição também foi sugerida por Bierman et al. (2012) para o mesmo tipo de problema abordado aqui.

Suponha que $n$ alíquotas de $1 \mathrm{~m}^{3}$ tenham sido colhidas aleatoriamente do tanque e que o número de organismos na alíquota $i, X_{i}$, segue uma distribuição Poisson com média $\lambda_{i}$. Assumindo que $\lambda_{i}$ segue uma distribuição Gama então $X_{i}$ segue uma distribuição Binomial Negativa. Neste caso o procedimento de teste para a hipótese (2.1) é

$$
\left\{\begin{array}{l}
\text { Rejeitar } H_{0}, \text { se } \sum_{i=1}^{n} X_{i} \geq c \\
\text { ou } \\
\text { Não rejeitar } H_{0}, \text { se } \sum_{i=1}^{n} X_{i}<c
\end{array}\right.
$$

em que $c$ é determinado usando (2.5) com

$$
\mathbb{P}\left[\sum_{i=1}^{n} X_{i}=x \mid \lambda, k\right]=\frac{\Gamma(n k+x)}{\Gamma(x+1) \Gamma(n k)}\left(\frac{\lambda}{\lambda+k}\right)^{x}\left(\frac{k}{\lambda+k}\right)^{n k}, \quad \lambda, k>0, \quad x=0,1,2, \ldots
$$

Consideramos valores $k=1,3$ e 25, que correspondem a diferentes distribuições da concentração de organismos com valor esperado $\lambda=10$ (Figura 2.11) para calcular o menor tamanho amostral $n$ 
para o qual o teste tem nível de significância $\leq a$ e poder $\geq 1-b$ para diferentes valores de $\lambda$. Um pseudo-algoritmo para a obtenção de $n$ está descrito no Apêndice D. Os resultados estão dispostos nas Tabelas 2.18, 2.19 e 2.20 .

Os procedimentos de teste apresentados neste capítulo são uniformemente mais poderosos [DeGroot (1975)], com exceção do caso para o qual utilizamos processos de Poisson não-homogêneos.

Usando os tamanhos amostrais apresentados nas Tabelas 2.19 e 2.20, foram simuladas contagens de organismos por meio de (2.14) considerando alíquotas de $1 \mathrm{~m}^{3}$ poder mínimo de $0.90(b=0.10)$, nível de significância de 0.05 e $\lambda=14 \mathrm{org} / \mathrm{m}^{3}$ ou $15 \mathrm{org} / \mathrm{m}^{3}$.

Tabela 2.18: Menor número ( $\mathrm{n}$ ) de alíquotas de $1 \mathrm{~m}^{3}$ para qual o poder do teste (2.15) é $\geq 1-b$ e o nível de significância é $\leq a(a=0.05)$, usando a distribuição Binomial Negativa com $k=1$.

\begin{tabular}{ccccccc}
\hline \hline \multirow{2}{*}{$\lambda$} & \multicolumn{3}{c}{$b=0.05$} & \multicolumn{3}{c}{$b=0.10$} \\
\cline { 2 - 7 } & $c$ & $n$ & $1-\beta(\lambda)$ & $c$ & $n$ & $1-\beta(\lambda)$ \\
\hline 11 & 13690 & 1307 & 0.9500 & 10871 & 1032 & 0.9001 \\
12 & 3892 & 357 & 0.9501 & 3106 & 282 & 0.9009 \\
13 & 1952 & 173 & 0.9502 & 1557 & 136 & 0.9009 \\
14 & 1234 & 106 & 0.9506 & 983 & 83 & 0.9011 \\
15 & 873 & 73 & 0.9504 & 707 & 58 & 0.9039 \\
\hline \hline
\end{tabular}

Tabela 2.19: Menor número ( $n$ ) de alíquotas de $1 \mathrm{~m}^{3}$ para qual o poder do teste (2.15) é $\geq 1-b$ e o nível de significância é $\leq a(a=0.05)$, usando a distribuição Binomial Negativa com $k=3$.

\begin{tabular}{ccccccc}
\hline \hline \multirow{2}{*}{$\lambda$} & \multicolumn{3}{c}{$b=0.05$} & \multicolumn{3}{c}{$b=0.10$} \\
\cline { 2 - 7 } & $c$ & $n$ & $1-\beta(\lambda)$ & $c$ & $n$ & $1-\beta(\lambda)$ \\
\hline 11 & 5368 & 513 & 0.9501 & 4271 & 406 & 0.9008 \\
12 & 1531 & 141 & 0.9516 & 1217 & 111 & 0.9009 \\
13 & 762 & 68 & 0.9503 & 612 & 54 & 0.9028 \\
14 & 482 & 42 & 0.9534 & 384 & 33 & 0.9043 \\
15 & 340 & 29 & 0.9527 & 274 & 23 & 0.9034 \\
\hline \hline
\end{tabular}

Tabela 2.20: Menor número ( $n$ ) de alíquotas de $1 \mathrm{~m}^{3}$ para qual o poder do teste (2.15) é $\geq 1-b$ e o nível de significância é $\leq a(a=0.05)$, usando a distribuição Binomial Negativa com $k=25$.

\begin{tabular}{ccccccc}
\hline \hline \multirow{2}{*}{$\lambda$} & \multicolumn{3}{c}{$b=0.05$} & \multicolumn{3}{c}{$b=0.10$} \\
\cline { 2 - 7 } & $c$ & $n$ & $1-\beta(\lambda)$ & $c$ & $n$ & $1-\beta(\lambda)$ \\
\hline 11 & 1710 & 164 & 0.9509 & 1361 & 130 & 0.9030 \\
12 & 482 & 45 & 0.9540 & 377 & 35 & 0.9017 \\
13 & 240 & 22 & 0.9531 & 197 & 18 & 0.9119 \\
14 & 154 & 14 & 0.9586 & 121 & 11 & 0.9098 \\
15 & 110 & 10 & 0.9634 & 88 & 8 & 0.9158 \\
\hline \hline
\end{tabular}

(a) $\lambda=14$.

Neste caso, considerando $k=3$ o tamanho amostral para alíquotas de $1 m^{3}$ é 33 . Valores simulados sob estas condições estão apresentados na Tabela 2.21. Considerando $\alpha=0.05$, 


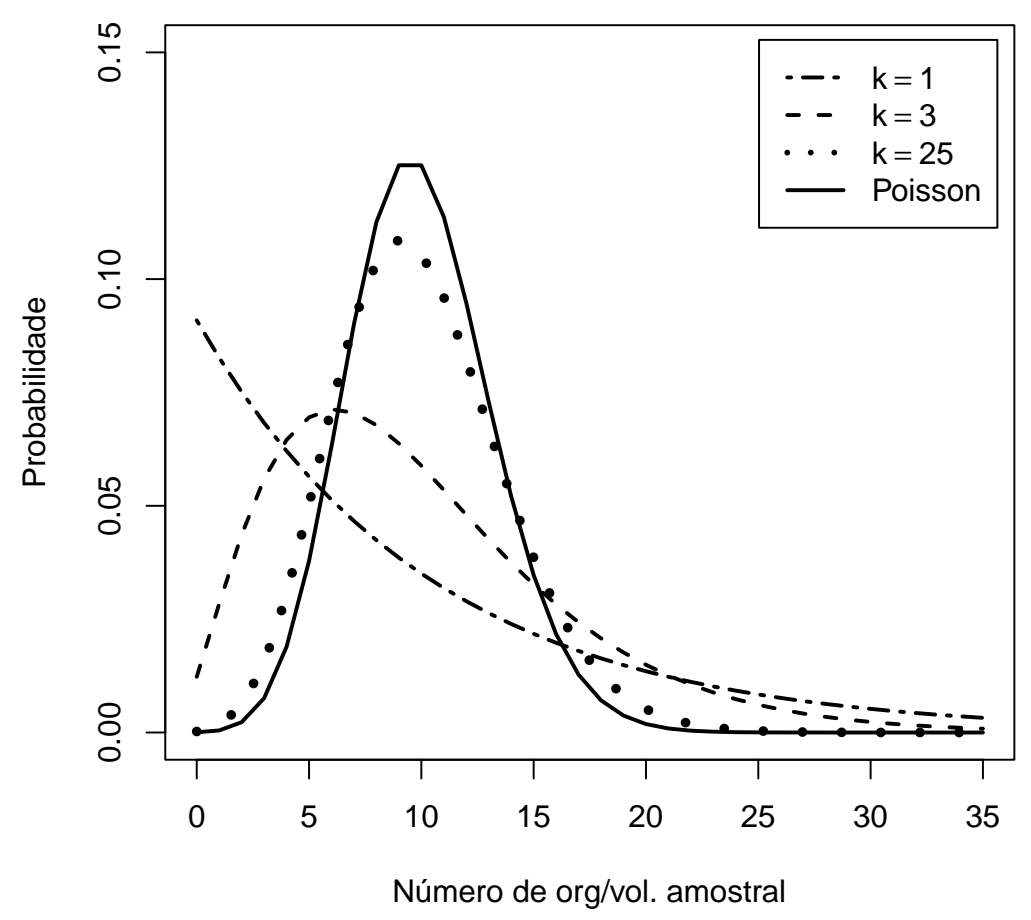

Figura 2.11: Distribuições Binomiais Negativas com média $\lambda=10$, e distribuição Poisson com média $\lambda=10$.

temos $c=384$ e como $\sum_{i=1}^{n} X_{i}=421>c$, concluimos que o tanque não cumpre a norma IMO. Considerando $k=25$ o tamanho amostral para alíquotas de $1 \mathrm{~m}^{3}$ é 11 . Valores simulados sob estas condições estão apresentados na Tabela 2.22. Considerando $\alpha=0.05$, temos $c=121 \mathrm{e}$ como $\sum_{i=1}^{n} X_{i}=129>c$, concluimos que o tanque não cumpre a norma IMO.

Tabela 2.21: Contagens de organismos simuladas usando (2.14) para $\lambda=14, k=3$ e $n=33$.

\begin{tabular}{ccccccccccc}
\hline \hline \multicolumn{11}{c}{ Contagens $\left(\mathrm{org} / \mathrm{m}^{3}\right)$} \\
\hline 0 & 2 & 16 & 4 & 7 & 12 & 28 & 4 & 17 & 3 & 12 \\
15 & 27 & 22 & 27 & 12 & 4 & 13 & 14 & 11 & 13 & 16 \\
16 & 12 & 16 & 18 & 14 & 3 & 13 & 28 & 4 & 5 & 13 \\
\hline \hline
\end{tabular}

Tabela 2.22: Contagens de organismos simuladas usando (2.14) para $\lambda=14, k=25$ e $n=11$.

\begin{tabular}{cccccc}
\hline \hline \multicolumn{6}{c}{ Contangens $\left(\mathrm{org} / \mathrm{m}^{3}\right)$} \\
\hline 4 & 6 & 8 & 11 & 12 & 21 \\
9 & 24 & 12 & 10 & 12 & - \\
\hline \hline
\end{tabular}

(b) $\lambda=15$.

Neste caso, considerando $k=3$ o tamanho amostral para alíquotas de $1 \mathrm{~m}^{3}$ é 23 . Valores 
simulados sob estas condições estão apresentados na Tabela 2.23. Considerando $\alpha=0.05$, temos $c=274$ e como $\sum_{i=1}^{n} X_{i}=250<c$, concluimos que o tanque cumpre a norma IMO. Considerando $k=25$ o tamanho amostral para alíquotas de $1 \mathrm{~m}^{3}$ é 8 . Valores simulados sob estas condições são $8,19,17,10,14,14,22$ e 9. Considerando $\alpha=0.05$, temos $c=88$ e como $\sum_{i=1}^{n} X_{i}=113>c$, concluimos que o tanque não cumpre a norma IMO.

Tabela 2.23: Contagens de organismos simuladas usando (2.14) para $\lambda=15, k=3$ e $n=23$.

\begin{tabular}{cccccccccccc}
\hline \hline \multicolumn{11}{c}{ Contanges $\left(\mathrm{org} / \mathrm{m}^{3}\right)$} \\
\hline 4 & 16 & 18 & 12 & 11 & 14 & 26 & 4 & 11 & 8 & 9 & 8 \\
9 & 6 & 21 & 1 & 7 & 17 & 19 & 2 & 12 & 1 & 14 & - \\
\hline \hline
\end{tabular}




\section{Capítulo 3}

\section{Avaliação do cumprimento das}

\section{normas IMO por meio de estimação}

\subsection{Introdução}

O procedimento baseado em testes de hipóteses é conveniente para tomada de decisões, porém não fornece estimativas sobre a magnitude da concentração média. Um enfoque em que ambas estas características são contempladas é o de estimação.

Considere $n$ alíquotas de $1 \mathrm{~m}^{3}$ colhidas aleatoriamente do tanque e sejam $X_{1}, X_{2}, \ldots, X_{n}$ os números de organismos nessas alíquotas. Suponha que o número esperado de organismos em cada alíquota de $1 \mathrm{~m}^{3}$ seja $\mathbb{E}\left[X_{i}\right]=\lambda$ e que a variância correspondente seja $\operatorname{Var}\left[X_{i}\right]=\sigma^{2}$. Um estimador natural para $\lambda$, é a média amostral $(\bar{X})$. O objetivo é determinar $n$ tal que

$$
\mathbb{P}[|\bar{X}-\lambda|<\epsilon]>1-\delta
$$

$\operatorname{com} \epsilon>0$ e $0<\delta<1$. Uma alternativa para calcular $n$ satisfazendo (3.1) é utilizar uma aproximação da distribuição de $\bar{X}$ pela distribuição Normal, obtendo

$$
n>\frac{\sigma_{X}^{2} z_{\delta / 2}^{2}}{\epsilon^{2}}
$$

em que $z_{\delta / 2}$ é o quantil de ordem $(1-\delta / 2)$ da distribuição Normal padrão. Um problema com (3.2) é o possível erro de aproximação e outro é que precisamos de uma estimativa da variância $\sigma_{X}^{2}$ para 
a obtenção do resultado. Outra alternativa é utilizar a desigualdade de Chebyshev, obtendo

$$
n>\frac{\sigma_{X}^{2}}{\epsilon^{2} \delta}
$$

Neste caso, também é necessário estimar a variância $\sigma_{X}^{2}$. Uma possível solução é considerar um modelo para a distribuição de probabilidades de $X$. Para problemas desta natureza, um modelo bastante utilizado é a distribuição Poisson.

\subsection{Tamanho amostral aproximado}

Chen (2008) deduz uma fórmula simples para calcular o tamanho amostral mínimo para estimar a média de uma distribuição Poisson baseado em um critério misto de limitação dos erros absoluto e relativo. O valor de $|\bar{X}-\lambda|$ é o erro absoluto; o erro relativo é $|\bar{X}-\lambda| / \lambda$. Por exemplo, se $\lambda=50$ e $\bar{X}=49$ então os erros absoluto e relativo são respectivamente $|\bar{X}-\lambda|=1$ e $|\bar{X}-\lambda| / \lambda=0.02$. Por outro lado se $\lambda=5$ e $\bar{X}=4$, os erros absoluto e relativo são, respectivamente, $|\bar{X}-\lambda|=1$ e $|\bar{X}-\lambda| / \lambda=0.2$.

Sob essa abordagem, não é necessário estimar nenhum parâmetro nem utilizar uma aproximação pela distribuição Normal; basta fixar o nível de confiança $(1-\delta)$ e os limites desejados para os erros absoluto e relativo.

Para aplicação ao problema sob investigação neste trabalho, inicialmente, reproduzimos os resultados de Chen (2008), admitindo que a concentração de organismos no tanque é homogênea e que o número de organismos por unidade de volume segue uma distribuição Poisson com média $\lambda$.

Teorema 3.1 (Chen, 2008). Seja $X_{i}(i=1, \ldots, n)$ uma amostra aleatória de uma distribuição Poisson com parâmetro $\lambda>0$. Seja $\widehat{\lambda}=n^{-1} \sum_{i=1}^{n} X_{i}$ um estimador para $\lambda$. Sejam $\epsilon_{a}, \epsilon_{r} \in(0,1)$ respectivamente os erros absoluto e relativo máximos e $\delta \in(0,1)$. Então, uma condição suficiente para que

$$
\mathbb{P}\left[|\widehat{\lambda}-\lambda|<\epsilon_{a} \text { ou }|\widehat{\lambda}-\lambda|<\epsilon_{r} \lambda\right]>1-\delta
$$

$e ́$

$$
n>\frac{\epsilon_{r}}{\epsilon_{a}} \times \frac{\log (2 / \delta)}{\left(1+\epsilon_{r}\right) \log \left(1+\epsilon_{r}\right)-\epsilon_{r}} .
$$

Para acomodar concentrações variáveis no tanque de lastro podemos utilizar uma distribuição Binomial Negativa e nesse caso, uma generalização do Teorema 3.1 é dada por

Teorema 3.2. Seja $X_{i}(i=1, \ldots, n)$ uma amostra aleatória de uma distribuição Binomial Negativa 
com parâmetros $\lambda$ e k. Seja $\widehat{\lambda}=n^{-1} \sum_{i=1}^{n} X_{i}$ um estimador para $\lambda$. Sejam $\epsilon_{a}>0, \epsilon_{r} \in(0,1)$ respectivamente os erros absoluto e relativo, e $\delta \in(0,1)$. Então, uma condição suficiente para que

$$
\mathbb{P}\left[|\widehat{\lambda}-\lambda|<\epsilon_{a} \text { ou }|\widehat{\lambda}-\lambda|<\epsilon_{r} \lambda\right]>1-\delta
$$

é

$$
n>\frac{\epsilon_{r}}{\epsilon_{a}} \times \frac{\log (2 / \delta)}{\left(1+\epsilon_{r}\right) \log \left(1+\epsilon_{r}\right)-\left(\frac{\epsilon_{r}}{\epsilon_{a}} k+1+\epsilon_{r}\right) \log \left(1+\frac{\epsilon_{a} \epsilon_{r}}{k \epsilon_{r}+\epsilon_{a}}\right)} .
$$

A demonstração está detalhada no Apêndice A.

Corolário 3.1. Seja $n_{B N}\left(n_{P}\right)$ o menor tamanho amostral satisfazendo o Teorema 3.2 (3.1). Então $n_{B N} \rightarrow n_{P}$ quando $k \rightarrow \infty$.

A demonstração está detalhada no Apêndice A.

Para calcular o valor de $n$ baseado no Teorema 3.2 é necessário fixar o valor de $k$. Como exemplo utilizamos os casos correspondentes às distribuições apresentadas na Figura 2.10. Alguns valores de $n$ podem ser encontrados nas Tabelas 3.1 e 3.2. Estudos de simulação mostram que os valores de $n$ obtidos por meio do Teorema 3.2 são bastante conservadores, ou seja, produzem níveis de confiança muito maiores que o limite $(1-\delta)$ fixado, chegando em alguns casos a produzir níveis de confiança muito próximos de 1. Métodos que forneçam resultados menos conservadores podem ser considerados.

Tabela 3.1: Tamanho amostral aproximado para estimar a concentração média $(\lambda)$ de organismos no tanque de lastro usando o Teorema $3.2(\delta=0.05)$.

\begin{tabular}{ccccc}
\hline \hline \multirow{2}{*}{$\lambda$} & \multirow{2}{*}{$\epsilon_{a}$} & \multicolumn{2}{c}{$n$} \\
\cline { 4 - 5 } & & & $\epsilon_{r}=0.05$ & $\epsilon_{r}=0.1$ \\
\hline 3 & 1 & 0.5 & 3350 & 940 \\
3 & 1 & 0.99 & 3201 & 864 \\
\hline 5 & 20 & 0.5 & 453 & 192 \\
5 & 20 & 0.99 & 304 & 117 \\
\hline 18 & 3 & 0.5 & 1317 & 415 \\
18 & 3 & 0.99 & 1168 & 340 \\
\hline 13 & 15 & 0.5 & 910 & 205 \\
13 & 15 & 0.99 & 762 & 130 \\
\hline 20 & 350 & 0.5 & 309 & 155 \\
20 & 350 & 0.99 & 161 & 80 \\
\hline \hline
\end{tabular}

\subsection{Tamanho amostral exato}

Chen $(2007,2011)$ estabelece um método de cálculo para determinar o tamanho amostral exato 
Tabela 3.2: Tamanho amostral aproximado para estimar a concentração média $(\lambda)$ de organismos no tanque de lastro usando o Teorema 3.2 $(\delta=0.01)$.

\begin{tabular}{ccccc}
\hline \hline \multirow{2}{*}{$\lambda$} & \multirow{2}{*}{$k$} & \multirow{2}{*}{$\epsilon_{a}$} & \multicolumn{2}{c}{$n$} \\
\cline { 4 - 5 } & & & $\epsilon_{r}=0.05$ & $\epsilon_{r}=0.1$ \\
\hline 3 & 1 & 0.5 & 4811 & 1349 \\
3 & 1 & 0.99 & 4598 & 1241 \\
\hline 5 & 20 & 0.5 & 650 & 276 \\
5 & 20 & 0.99 & 437 & 168 \\
\hline 18 & 3 & 0.5 & 1891 & 596 \\
18 & 3 & 0.99 & 1678 & 488 \\
\hline 13 & 15 & 0.5 & 723 & 295 \\
13 & 15 & 0.99 & 510 & 186 \\
\hline 20 & 350 & 0.5 & 444 & 223 \\
20 & 350 & 0.99 & 231 & 114 \\
\hline \hline
\end{tabular}

mínimo para estimar a média de distribuições Poisson e Binomial com nível de confiança prescrito, controlando o erro absoluto, ou o erro relativo, ou ambos, sendo apenas necessário a fixação de um intervalo que contenha a média. Seguindo a ideia de Chen $(2007,2011)$ estendemos tais resultados para o caso em que se deseja estimar a média de uma distribuição Binomial Negativa com a finalidade de aplicá-lo ao problema de estimação da concentração de organismos em um tanque de lastro.

\subsubsection{Fixação de limite apenas para o erro absoluto}

Considere uma amostra aleatória, $\left(X_{1}, X_{2}, \ldots, X_{n}\right)$, que representa as contagens de organismos em um tanque de lastro, assumindo que tais contagens seguem uma distribuição Binomial Negativa com média $\lambda$ e parâmetro de dispersão $k$. Seja $\epsilon_{a}$ o erro absoluto máximo que se deseja obter ao estimar o parâmetro $\lambda$ por meio do estimador $\widehat{\lambda}=n^{-1} \sum_{i=1}^{n} X_{i}$. Neste caso desejamos obter o valor de $n$ tal que

$$
\mathbb{P}\left[|\widehat{\lambda}-\lambda|<\epsilon_{a}\right]>1-\delta
$$

em que $\lambda \in[a, b]$ com $a$ e $b$ indicando limites pré-fixados e $\delta \in(0,1)$. Com essa finalidade, demonstramos o seguinte resultado a partir da ideia de Chen (2011).

Teorema 3.3. Sejam $\epsilon_{a}>0,0<a<b<\infty$ e $X_{1}, X_{2}, \ldots, X_{n}$ uma amostra aleatória de uma distribuição Binomial Negativa $(\lambda, k)$ com $\lambda \in[a, b]$ em que a e $b$ são constantes conhecidas. Seja $\widehat{\lambda}=n^{-1} \sum_{i=1}^{n} X_{i}$. Então, o mínimo de $\mathbb{P}\left[|\widehat{\lambda}-\lambda|<\epsilon_{a}\right]$ com $\lambda \in[a, b]$ é atingido no conjunto $\{a, b\} \cup\left\{\ell / n+\epsilon_{a} \in(a, b): \ell \in \mathbb{Z}\right\} \cup\left\{\ell / n-\epsilon_{a} \in(a, b): \ell \in \mathbb{Z}\right\}$, que possui menos que $2 n(b-a)+4$ elementos.

A demonstração está detalhada no Apêndice B. O Teorema 3.3 estabelece uma maneira para 
o cálculo do valor de $n$ fixando-se a priori o limite para erro absoluto máximo $\left(\epsilon_{a}\right)$, o nível de confiança mínimo $(1-\delta)$ e um intervalo que contenha a média $\lambda([a, b])$. Um pseudo-algoritmo explicando o cálculo do $n$ usando o Teorema 3.3 encontra-se no Apêndice D.

\subsubsection{Fixação de limite apenas para o erro relativo}

Seja $\epsilon_{r} \in(0,1)$ o erro relativo máximo que se deseja obter ao estimar o parâmetro $\lambda$ com o estimador $\hat{\lambda}$. Então, desejamos obter o valor de $n$ tal que

$$
\mathbb{P}\left[|\widehat{\lambda}-\lambda| / \lambda<\epsilon_{r}\right]>1-\delta
$$

em que $\lambda \in[a, b]$ com $a$ e $b$ indicando limites pré-fixados e $\delta \in(0,1)$. Com essa finalidade, demonstramos o seguinte resultado a partir da ideia de Chen (2011).

Teorema 3.4. Sejam $0<\epsilon_{r}<1,0<a<b<\infty$ e $X_{1}, X_{2}, \ldots, X_{n}$ uma amostra aleatória de uma distribuição Binomial Negativa $(\lambda, k)$ com $\lambda \in[a, b]$ em que a e $b$ são constantes conhecidas. Seja $\widehat{\lambda}=n^{-1} \sum_{i=1}^{n} X_{i}$. Então, o mínimo de $\mathbb{P}\left[|\widehat{\lambda}-\lambda| / \lambda<\epsilon_{r}\right]$ com relação a $\lambda \in[a, b]$ é atingido no conjunto $\{a, b\} \cup\left\{\ell /\left(n\left(1-\epsilon_{r}\right)\right) \in(a, b): \ell \in \mathbb{Z}\right\} \cup\left\{\ell /\left(n\left(1+\epsilon_{r}\right)\right) \in(a, b): \ell \in \mathbb{Z}\right\}$, que possui menos que $2 n(b-a)+4$ elementos.

A demonstração está detalhada no Apêndice C. Um pseudo-algoritmo explicando o cálculo do $n$ usando o Teorema 3.4 encontra-se no Apêndice D.

\subsubsection{Fixação de limites para os erros abosoluto e relativo}

Neste caso consideramos limites para ambos os erros absoluto e relativo. O objetivo é calcular $n$ tal que

$$
\mathbb{P}\left[|\widehat{\lambda}-\lambda|<\epsilon_{a} \text { ou }|\widehat{\lambda}-\lambda| / \lambda<\epsilon_{r}\right]>1-\delta
$$

em que $\lambda \in[a, b]$ com $a$ e $b$ indicando limites pré-fixados e $\delta \in(0,1)$. Com essa finalidade, demonstramos o seguinte resultado a partir da ideia de Chen (2011).

Teorema 3.5. Sejam $\epsilon_{a}>0,0<\epsilon_{r}<1,0<a<\epsilon_{a} / \epsilon_{r}<b<\infty$ e $X_{1}, X_{2}, \ldots, X_{n}$ uma amostra aleatória de uma distribuição Binomial Negativa $(\lambda, k) \operatorname{com} \lambda \in[a, b]$. Seja $\hat{\lambda}=n^{-1} \sum_{i=1}^{n} X_{i}$. Então, o mínimo de $\mathbb{P}\left[|\widehat{\lambda}-\lambda|<\epsilon_{a}\right.$ ou $\left.|\widehat{\lambda}-\lambda| / \lambda<\epsilon_{r}\right]$ com $\lambda \in[a, b]$ é alcançado no conjunto $\left\{a, b, \epsilon_{a} / \epsilon_{r}\right\} \cup\left\{\ell / n+\epsilon_{a} \in\left(a, \epsilon_{a} / \epsilon_{r}\right): \ell \in \mathbb{Z}\right\} \cup\left\{\ell / n-\epsilon_{a} \in\left(\epsilon_{a} / \epsilon_{r}, b\right): \ell \in \mathbb{Z}\right\} \cup\left\{\ell /\left(n\left(1+\epsilon_{r}\right)\right) \in\right.$ $\left.\left(a, \epsilon_{a} / \epsilon_{r}\right): \ell \in \mathbb{Z}\right\} \cup\left\{\ell /\left(n\left(1-\epsilon_{r}\right)\right) \in\left(\epsilon_{a} / \epsilon_{r}, b\right): \ell \in \mathbb{Z}\right\}$, que possui menos que $2 n(b-a)+7$ elementos. 
A demonstração está detalhada no Apêndice C. Um pseudo-algoritmo explicando o cálculo do $n$ usando o Teorema 3.5 encontra-se no Apêndice D.

Uma rotina escrita em $\mathrm{R}$ elaborada para calcular o tamanho amostral por meio dos Teoremas 3.3, 3.4 e 3.5 pode ser obtida em www.ime.usp.br/ ${ }^{\sim}$ eliardo. Para calcular o valor de $n$ por meio desses resultados é necessário fixar o valor de $k$ e o intervalo $[a, b]$. Como exemplo consideramos os casos descritos na Figura 2.10 em que para cada valor de $k$ os valores de $a$ e $b$ são os quantis $1 \%$ e $99 \%$, respectivamente. Uma das razões para isto é que no caso onde $\lambda=5$ e $k=20$, a distribuição da concentração de organismos tem média em torno de 5 e valores acima de 10 já apresentam probabilidade quase nula. Na Tabela 3.3 encontram-se os intervalos adotados para cada caso da Figura 2.10. Nas Tabelas 3.4, 3.5, 3.6 e 3.7 encontram-se alguns valores de $n$ correspondentes aos Teoremas 3.3, 3.4 e 3.5. Alguns valores nas Tabelas 3.6 e 3.7 não foram calculados pois em tais casos a restrição $a<\epsilon_{a} / \epsilon_{r}<b$ não é respeitada.

Tabela 3.3: Intervalos $[a ; b]$ fixados para o cálculo do tamanho amostral exato para os casos apresentados na Figura 2.10.

\begin{tabular}{cccc}
\hline \hline$\lambda$ & $k$ & $a$ & $b$ \\
\hline 3 & 1 & 0.03 & 13.82 \\
5 & 20 & 2.77 & 7.97 \\
13 & 15 & 6.47 & 22.06 \\
18 & 3 & 2.61 & 50.44 \\
20 & 350 & 17.58 & 22.58 \\
\hline \hline
\end{tabular}

Tabela 3.4: Tamanho amostral exato para estimar a concentração média de organismos no tanque de lastro usando o Teorema 3.3 .

\begin{tabular}{ccccc}
\hline \hline \multirow{2}{*}{$\lambda$} & \multirow{2}{*}{$k$} & \multirow{2}{*}{$\epsilon_{a}$} & \multicolumn{2}{c}{$n$} \\
\cline { 4 - 5 } & & $\delta=0.05$ & $\delta=0.01$ \\
\hline 3 & 1 & 0.5 & 3147 & 5436 \\
3 & 1 & 0.99 & 803 & 1387 \\
\hline 5 & 20 & 0.5 & 173 & 298 \\
5 & 20 & 0.99 & 44 & 76 \\
\hline 18 & 3 & 0.5 & 13806 & 23845 \\
18 & 3 & 0.99 & 3522 & 6083 \\
\hline 13 & 15 & 0.5 & 840 & 1448 \\
13 & 15 & 0.99 & 215 & 370 \\
\hline 20 & 350 & 0.5 & 372 & 640 \\
20 & 350 & 0.99 & 95 & 164 \\
\hline \hline
\end{tabular}

Esses resultados podem ser aplicados ao problema de estimação da concentração de organismos em um tanque de lastro. Uma amostra $\left(x_{1}, x_{2}, \ldots, x_{n}\right)$ com o número de organismos em cada uma das $n$ alíquotas de $1 \mathrm{~m}^{3}$ coletadas do tanque de lastro poderá ser usada para estimar a concentração média. Supondo que o número de organismos viáveis em cada alíquota segue uma 
Tabela 3.5: Tamanho amostral exato para estimar a concentração média de organismos no tanque de lastro usando o Teorema 3.4.

\begin{tabular}{ccccc}
\hline \hline \multirow{2}{*}{$\lambda$} & \multirow{2}{*}{$k$} & \multirow{2}{*}{$\epsilon_{r}$} & \multicolumn{2}{c}{$n$} \\
\cline { 4 - 5 } & & & $\delta=0.05$ & $\delta=0.01$ \\
\hline 3 & 1 & 0.05 & 53067 & 91192 \\
3 & 1 & 0.1 & 13395 & 22885 \\
\hline 5 & 20 & 0.05 & 635 & 1097 \\
5 & 20 & 0.1 & 161 & 276 \\
\hline 18 & 3 & 0.05 & 1106 & 1907 \\
18 & 3 & 0.1 & 278 & 478 \\
\hline 13 & 15 & 0.05 & 342 & 590 \\
13 & 15 & 0.1 & 86 & 149 \\
\hline 20 & 350 & 0.05 & 93 & 160 \\
20 & 350 & 0.1 & 24 & 41 \\
\hline \hline
\end{tabular}

Tabela 3.6: Tamanho amostral exato para estimar a concentração média de organismos no tanque de lastro usando o Teorema $3.5(\delta=0.05)$.

\begin{tabular}{ccccc}
\hline \hline \multirow{2}{*}{$\lambda$} & \multirow{2}{*}{$*$} & \multirow{2}{*}{$\epsilon_{a}$} & \multicolumn{2}{c}{$n$} \\
\cline { 4 - 5 } & & $\epsilon_{r}=0.05$ & $\epsilon_{r}=0.1$ \\
\hline 3 & 1 & 0.5 & 1692 & 463 \\
3 & 1 & 0.99 & - & 424 \\
\hline 5 & 20 & 0.5 & - & 98 \\
5 & 20 & 0.99 & - & - \\
\hline 18 & 3 & 0.5 & 668 & 357 \\
18 & 3 & 0.99 & 591 & 290 \\
\hline 13 & 15 & 0.5 & 259 & - \\
13 & 15 & 0.99 & 181 & 66 \\
\hline 20 & 350 & 0.5 & - & - \\
20 & 350 & 0.99 & 83 & - \\
\hline \hline
\end{tabular}

distribuição Binomial Negativa com média $\lambda$ e parâmetro de dispersão $k$, o estimador de máxima verossimilhança para $\lambda$ é $\widehat{\lambda}=\bar{x}$, a média amostral, e o estimador de máxima verossimilhança para $k$ é obtido por meio da solução da equação

$$
-\psi(\widehat{k})+n \log \left(\frac{\widehat{k}}{\widehat{\lambda}+\widehat{k}}\right)+\sum_{i=1}^{n} \psi\left(\widehat{k}+x_{i}\right)=0,
$$

que pode ser obtida por meio de métodos de otimização, em que $\psi(x)=\Gamma^{\prime}(x) / \Gamma(x)$ é a função digama. Com essa finalidade utilizamos a função fitdistr do pacote MASS do software R [R Development Core Team (2012)], baseada no método BFGS de otimização [Broyden (1970); Fletcher (1970); Goldfarb (1970); Shanno (1970)]. O erro padrão dos estimadores de máxima verossimilhança é calculado por intermédio da informação de Fisher e o intervalo de confiança é estabelecido a partir da normalidade assintótica do estimador de máxima verossimilhança [Ver Sen et al. (2009), por exemplo]. 
Tabela 3.7: Tamanho amostral exato para estimar a concentração média de organismos no tanque de lastro usando o Teorema $3.5(\delta=0.01)$.

\begin{tabular}{ccccc}
\hline \hline \multirow{2}{*}{$\lambda$} & \multirow{2}{*}{$k$} & \multirow{2}{*}{$\epsilon_{a}$} & \multicolumn{2}{c}{$n$} \\
\cline { 4 - 5 } & & & $\epsilon_{r}=0.05$ & $\epsilon_{r}=0.1$ \\
\hline 3 & 1 & 0.5 & 2923 & 800 \\
3 & 1 & 0.99 & - & 733 \\
\hline 5 & 20 & 0.5 & - & 168 \\
5 & 20 & 0.99 & - & - \\
\hline 18 & 3 & 0.5 & 1153 & 207 \\
18 & 3 & 0.99 & 1021 & 168 \\
\hline 13 & 15 & 0.5 & 445 & - \\
13 & 15 & 0.99 & 312 & 113 \\
\hline 20 & 350 & 0.5 & - & - \\
20 & 350 & 0.99 & 143 & - \\
\hline \hline
\end{tabular}

Para testar a hipótese (2.1) pode-se adotar o seguinte procedimento: se o limite inferior do intervalo de confiança for maior que 10, o tanque de lastro não cumpre a norma IMO; em caso contrário, o tanque de lastro cumpre a norma IMO.

Como ilustração, consideramos novamente os casos descritos na Figura 2.10 e os intervalos $(a, b)$ na Tabela 3.3. Para cada intervalo foram selecionados quatro valores diferentes para o parâmetro $\lambda$. Em cada um desses cenários, uma amostra de tamanho $n$ (obtido das Tabelas 3.4 e 3.5) foi simulada segundo essas especificações. Posteriormente, foram calculados os estimadores de máxima verossimilhança de $\lambda$ e $k$ e os respectivos intervalos de confiança. Consideramos os seguintes casos.

(a) $\lambda=3, k=1$

O tamanho amostral utilizado foi $n=803$ (Tabela 3.4$)$ e os limites para $\lambda$ foram $(0.03 ; 13.82)$ conforme a Tabela 3.3. O primeiro gráfico da Figura 3.1 apresenta estimativas de máxima verossimilhança $\left(\widehat{\lambda}_{i}\right)$ e respectivos intervalos de confiança (95\%). A linha tracejada representa a reta com 45 graus de inclinação. O segundo gráfico da Figura 3.1, apresenta estimativas de máxima verossimilhança de $k(\widehat{k})$ e seus respectivos intervalos de confiança $(95 \%)$. Na Figura 3.2 os círculos correspondem ao gráfico da distribuição Binomial Negativa com média $\lambda_{i}(i=1,2,3,4)$ e parâmetro de dispersão $k$; a linha tracejada indica a distribuição ajustada com base nas estimativas de máxima verossimilhança $\left(\widehat{\lambda}_{i}\right.$ e $\left.\widehat{k}\right)$. Note que em todos os casos, os intervalos de confiança contêm os verdadeiros valores dos parâmetros $\lambda_{i}$ e $k$ (Tabela 3.8).

(b) $\lambda=5, k=20$

O tamanho amostral utilizado foi $n=44$ (Tabela 3.4 ) e os limites para $\lambda$ foram $(2.77 ; 7.97$ ) conforme a Tabela 3.3. Note que exceto em um caso o intervalo de confiança não contém o verdadeiro valo do parâmetro $k$, nos demais os intervalos contêm (Figura 3.3). Somente o 
Tabela 3.8: Estimativas para os parâmetros do modelo (2.14) calculadas com base em uma amostra de tamanho $n=803$ simulada de uma distribuição Binomial Negativa com média $\lambda_{i}$ e $k=1$.

\begin{tabular}{c|ccc|cc}
\hline \hline$i$ & $\lambda_{i}$ & $\widehat{\lambda}_{i}$ & IC $(95 \%)$ & $\widehat{k}$ & IC $(95 \%)$ \\
\hline 1 & 1 & 0.951 & {$[0.855 ; 1.048]$} & 0.914 & {$[0.693 ; 1.135]$} \\
2 & 5 & 4.844 & {$[4.476 ; 5.123]$} & 0.997 & {$[0.871 ; 1.122]$} \\
3 & 9 & 8.922 & {$[8.288 ; 9.555]$} & 1.060 & {$[0.942 ; 1.178]$} \\
4 & 13 & 13.101 & {$[12.183 ; 14.019]$} & 1.052 & {$[0.942 ; 1.161]$} \\
\hline \hline
\end{tabular}
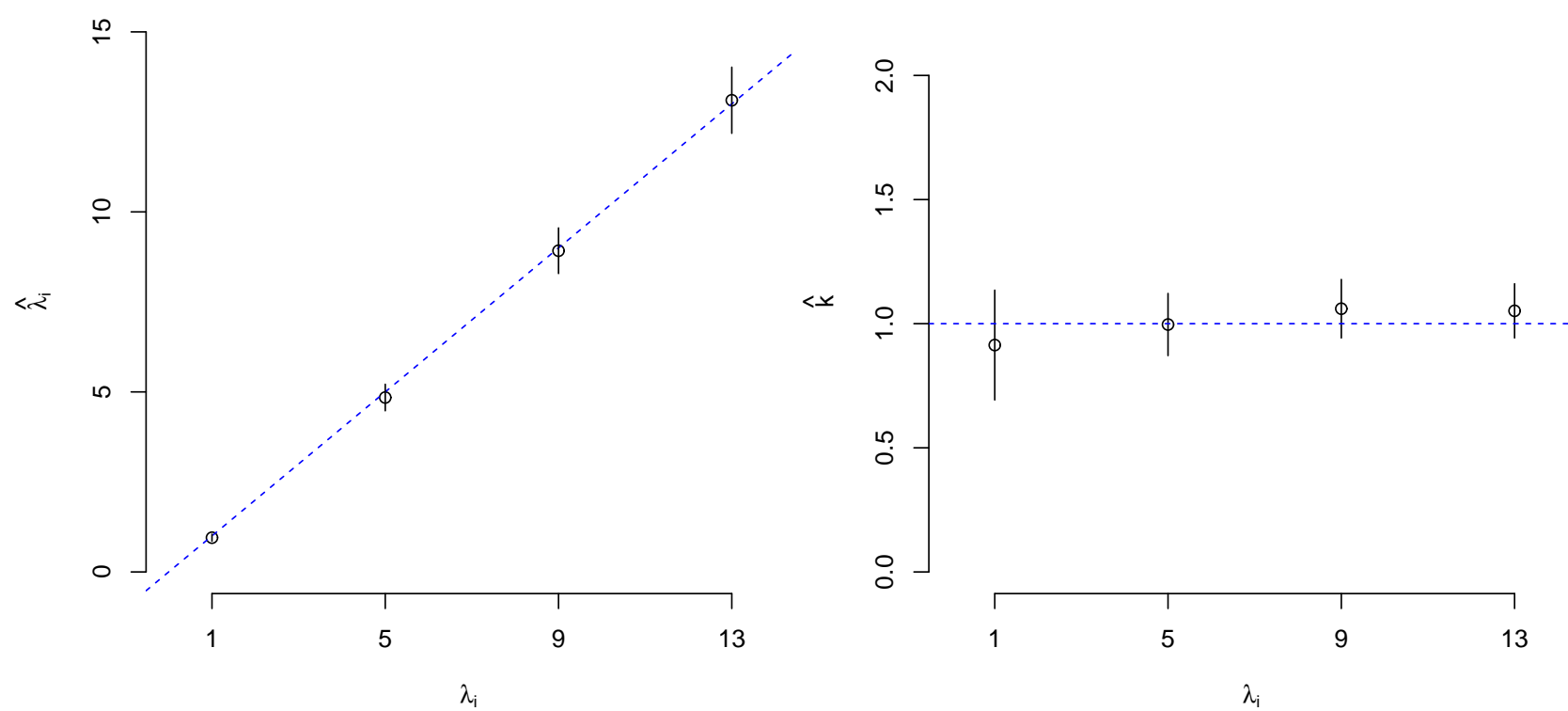

Figura 3.1: Estimativas de máxima verossimilhança $\left(\hat{\lambda}_{i} e \hat{k}\right)$ e intervalos de confiança calculados com base em uma amostra $(n=803)$ de uma Binomial Negativa com média $\lambda_{i}$ e dispersão $k$.

quarto intervalo de confiança para $k$ apresentou uma grande amplitude (Figura 3.3 e Tabela 3.9). As distribuições ajustadas estão bem próximas das distribuições teóricas mesmo no caso que apresentou intervalo de confiança para $k$ com grande amplitude (Figura 3.4).

(c) $\lambda=18, k=3$

O tamanho amostral utilizado foi $n=278$ (Tabela 3.5 ) e os limites para $\lambda$ foram $(2.61 ; 50.44$ ) conforme a Tabela 3.3. Note que em todos os casos, os intervalos de confiança contêm os verdadeiros valores dos parâmetros $\lambda_{i}$ e $k$ (Figura 3.5 e Tabela 3.10). As distribuições ajustadas estão bem próximas das distribuições teóricas (Figura 3.6).

(d) $\lambda=13, k=15$

O tamanho amostral utilizado foi $n=86$ (Tabela 3.5 ) e os limites para $\lambda$ foram $(6.47 ; 22.06$ ) conforme a Tabela 3.3. Note que em todos os casos, os intervalos de confiança contêm os ver- 


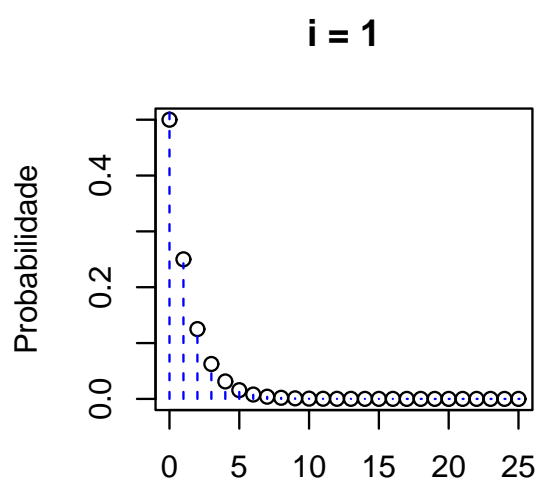

$\mathrm{X}$

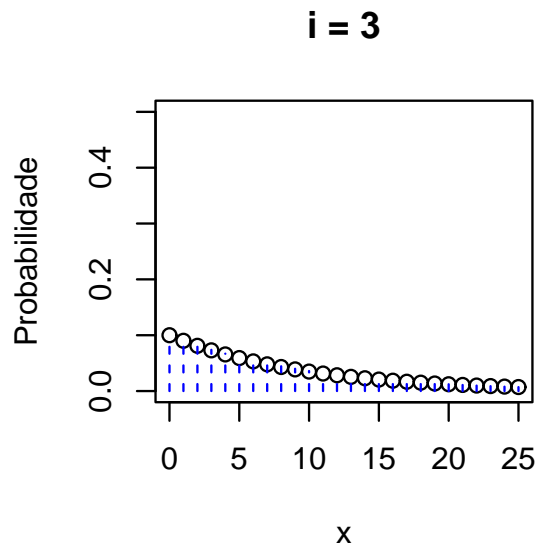

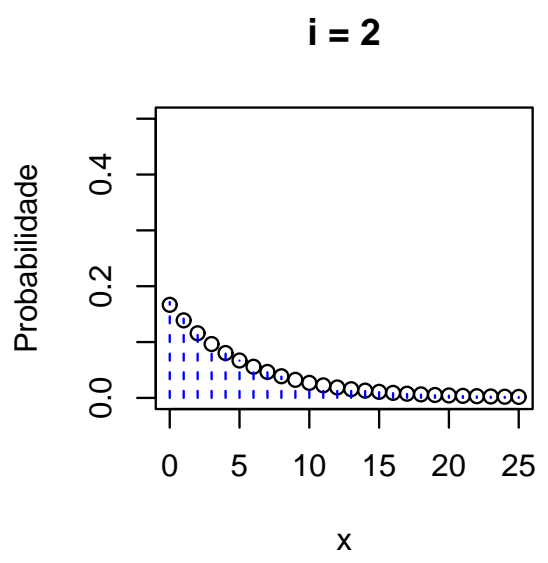

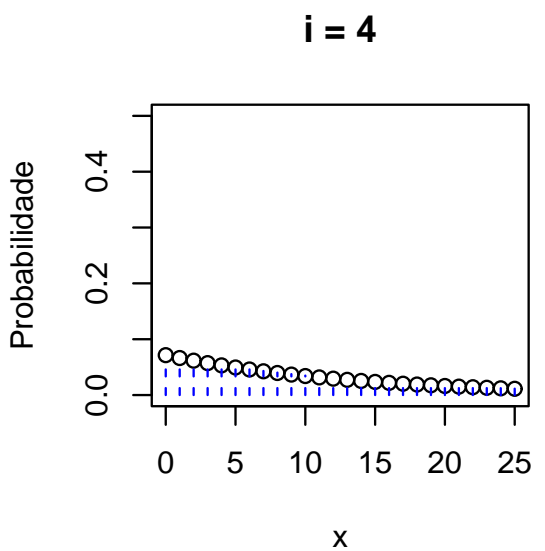

Figura 3.2: Distribuições de probabilidade teóricas com paramêtros $\lambda_{i}$ e $k$ (círculos) e distribuições ajustadas (linhas tracejadas).

dadeiros valores dos parâmetros $\lambda_{i}$ e $k$ (Figura 3.7 e Tabela 3.11). O primeiro intervalo de confiança para $k$ apresenta uma amplitude bastante grande (Figura 3.7). As distribuições ajustadas estão bem próximas das distribuições teóricas mesmo no caso que apresentou intervalo de confiança para $k$ com aplitude grande (Figura 3.8).

(e) $\lambda=20, k=350$

O tamanho amostral utilizado foi $n=803$ (Tabela 3.5) e os limites para $\lambda$ foram $(17.58 ; 22.58$ ) conforme a Tabela 3.3. Note que em apenas um caso o intervalo de confiança não contém o verdadeiro valor dos parâmetros $\lambda_{i}$ e $k$ (Figura 3.9 e Tabela 3.12). Todos os intervalos de confiança para $k$ apresentam amplitude bastande grande (Figura 3.9), entretanto as distribuições ajustadas estão bem próximas das distribuições teóricas (Figura 3.10). 
Tabela 3.9: Estimativas para os parâmetros do modelo (2.14) calculadas com base em uma amostra de tamanho $n=44$ simulada de uma distribuição Binomial Negativa com média $\lambda_{i}$ e $k=20$.

\begin{tabular}{c|ccc|cc}
\hline \hline$i$ & $\lambda_{i}$ & $\widehat{\lambda}_{i}$ & IC $(95 \%)$ & $\widehat{k}$ & IC $(95 \%)$ \\
\hline 1 & 3 & 2.5 & {$[1.934 ; 3.066]$} & 5.335 & {$[0 ; 12.773]$} \\
2 & 4.333 & 3.818 & {$[3.139 ; 4.497]$} & 9.978 & {$[0 ; 24.241]$} \\
3 & 5.667 & 5.477 & {$[4.677 ; 6.277]$} & 16.173 & {$[0 ; 42.789]$} \\
4 & 7 & 7.023 & {$[6.231 ; 7.814]$} & 318.041 & {$[0 ; 1540.963]$} \\
\hline \hline
\end{tabular}

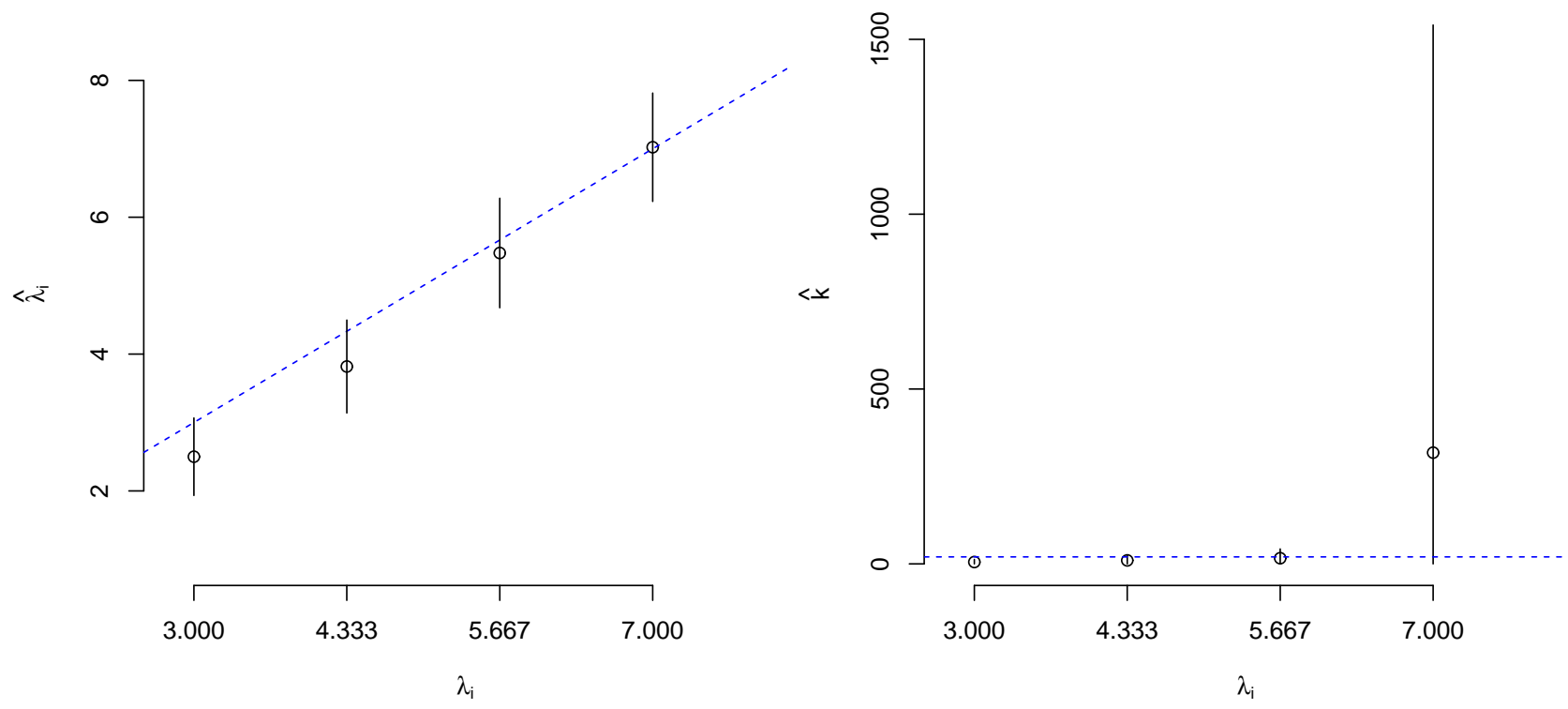

Figura 3.3: Estimativas de máxima verossimilhança $\left(\hat{\lambda}_{i}\right.$ e $\left.\hat{k}\right)$ e intervalos de confiança calculados com base em uma amostra aleatória de tamanho $n=44(\lambda=5, k=20)$.

Tabela 3.10: Estimativas para os parâmetros do modelo (2.14) calculadas com base em uma amostra de tamanho $n=278$ simulada de uma distribuição Binomial Negativa com média $\lambda_{i}$ e $k=3$.

\begin{tabular}{c|ccc|cc}
\hline \hline$i$ & $\lambda_{i}$ & $\widehat{\lambda}_{i}$ & IC $(95 \%)$ & $\widehat{k}$ & IC $(95 \%)$ \\
\hline 1 & 3 & 2.914 & {$[2.653 ; 3.174]$} & 4.236 & {$[2.430 ; 6.042]$} \\
2 & 18.667 & 18.871 & {$[17.406 ; 20.335]$} & 2.610 & {$[2.123 ; 3.098]$} \\
3 & 34.333 & 33.432 & {$[30.984 ; 35.879]$} & 2.794 & {$[2.308 ; 3.281]$} \\
4 & 50 & 49.489 & {$[45.998 ; 52.981]$} & 2.941 & {$[2.442 ; 3.441]$} \\
\hline \hline
\end{tabular}

Tabela 3.11: Estimativas para os parâmetros do modelo (2.14) calculadas com base em uma amostra de tamanho $n=86$ simulada de uma distribuição Binomial Negativa com média $\lambda_{i}$ e $k=15$.

\begin{tabular}{c|ccc|cc}
\hline \hline$i$ & $\lambda_{i}$ & $\widehat{\lambda}_{i}$ & IC $(95 \%)$ & $\widehat{k}$ & IC $(95 \%)$ \\
\hline 1 & 7 & 6.674 & {$[6.084 ; 7.265]$} & 39.627 & {$[0 ; 119.697]$} \\
2 & 12 & 11.593 & {$[10.541 ; 12.645]$} & 10.196 & {$[4.282 ; 16.110]$} \\
3 & 17 & 17.047 & {$[15.677 ; 18.416]$} & 11.655 & {$[5.7 ; 17.610]$} \\
4 & 22 & 22.907 & {$[21.506 ; 24.308]$} & 24.939 & {$[9.403 ; 40.475]$} \\
\hline \hline
\end{tabular}




$$
\mathbf{i}=\mathbf{1}
$$

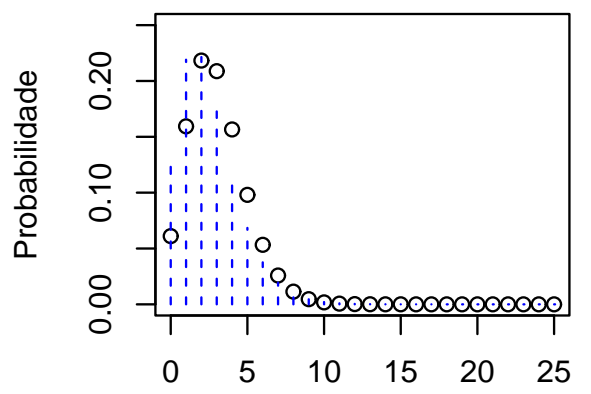

$\mathrm{X}$

$$
\mathbf{i}=\mathbf{3}
$$

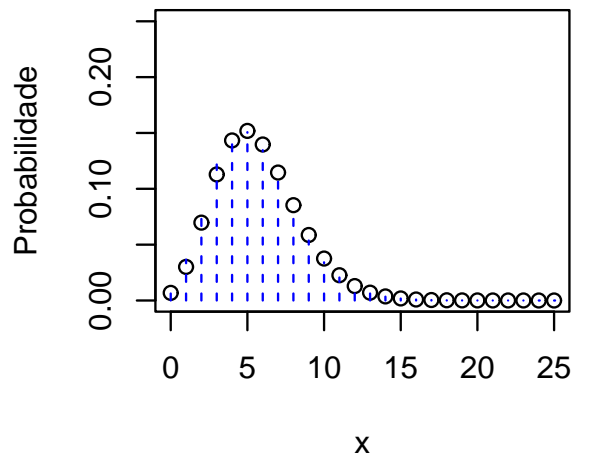

$\mathbf{i}=\mathbf{2}$

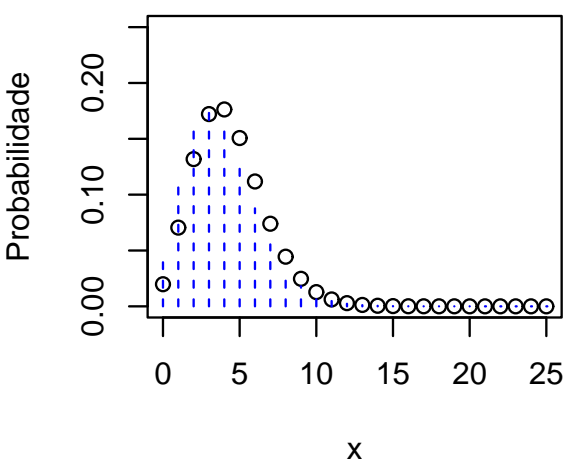

$\mathbf{i}=\mathbf{4}$

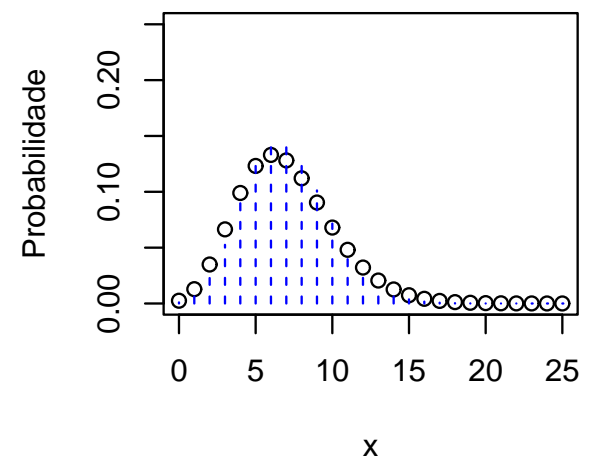

Figura 3.4: Distribuições de probabilidade com paramêtros $\lambda_{i}$ e $k$ (círculos) e distribuições ajustadas (linhas tracejadas) $(\lambda=5, k=20)$.
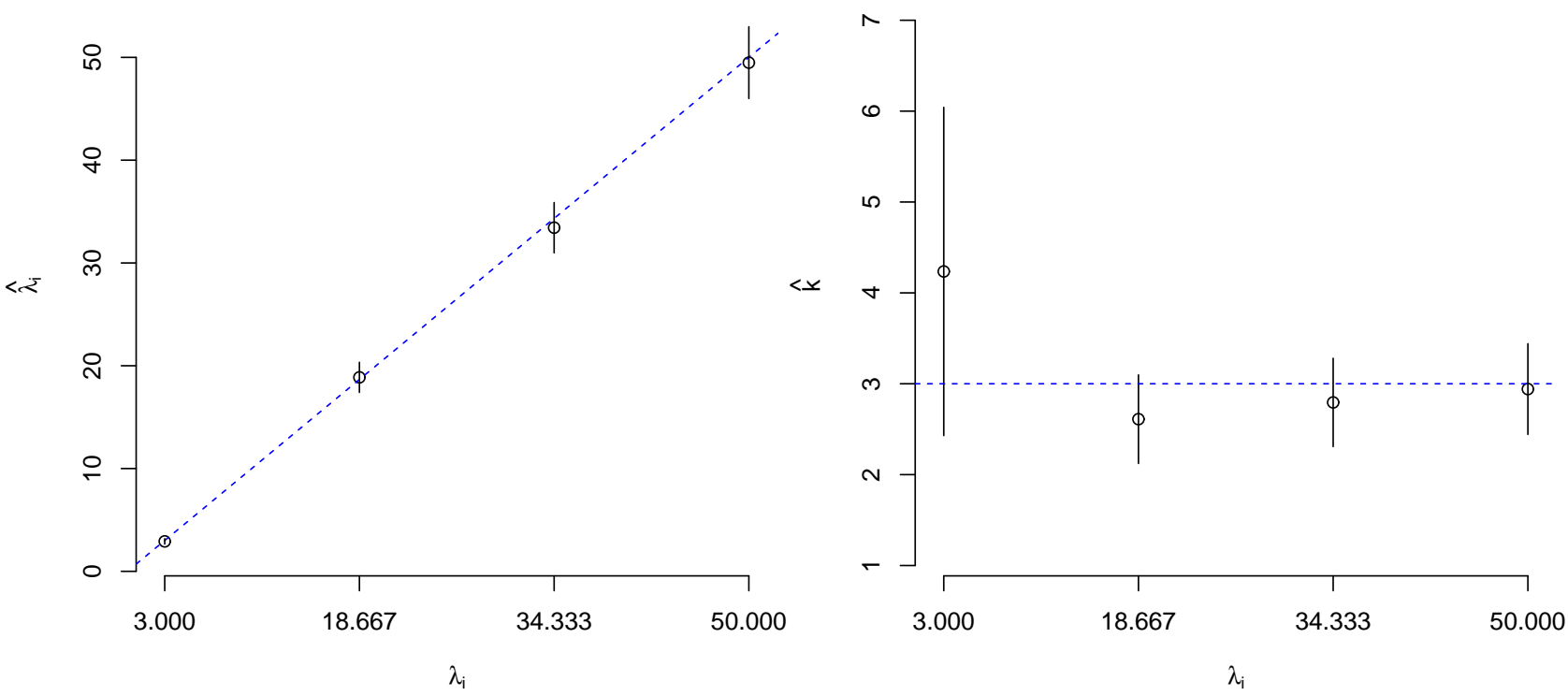

Figura 3.5: Estimativas de máxima verossimilhança $\left(\hat{\lambda_{i}}\right.$ e $\left.\hat{k}\right)$ e intervalos de confiança calculados com base em uma amostra $(n=278)$ de uma Binomial Negativa com média $\lambda_{i}$ e dispersão $k$. 
$\mathbf{i}=\mathbf{1}$

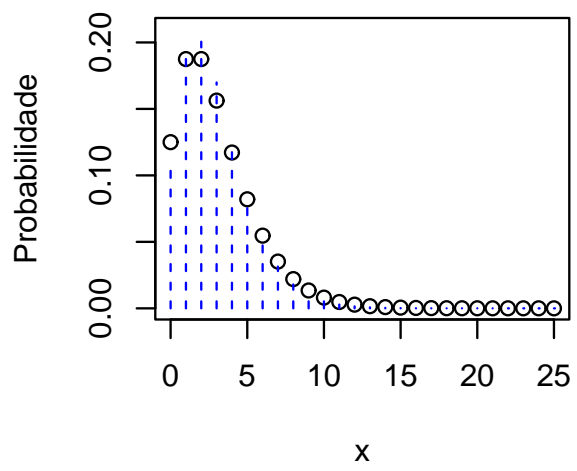

$\mathbf{i}=\mathbf{3}$

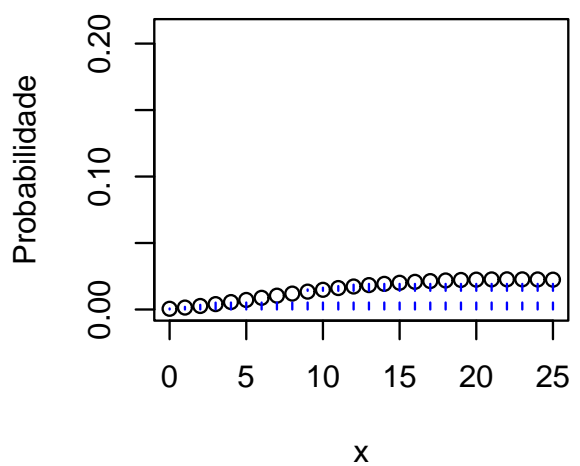

$\mathbf{i}=\mathbf{2}$

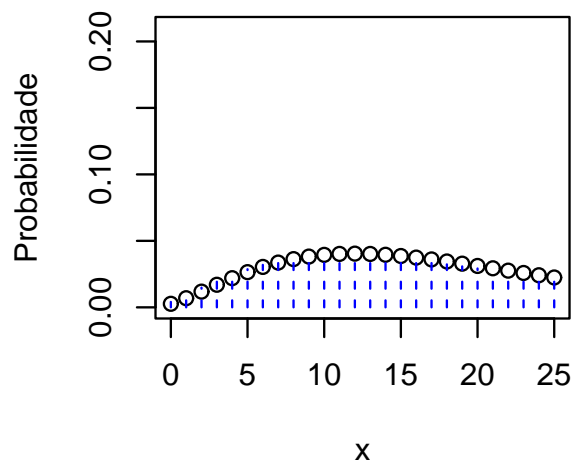

$\mathbf{i}=\mathbf{4}$

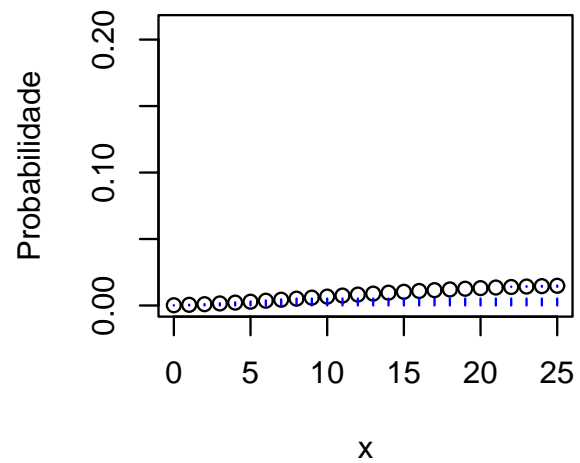

Figura 3.6: Distribuições de probabilidade com paramêtros $\lambda_{i}$ e $k$ (círculos) e distribuições ajustadas (linhas tracejadas) $(\lambda=18, k=3)$.
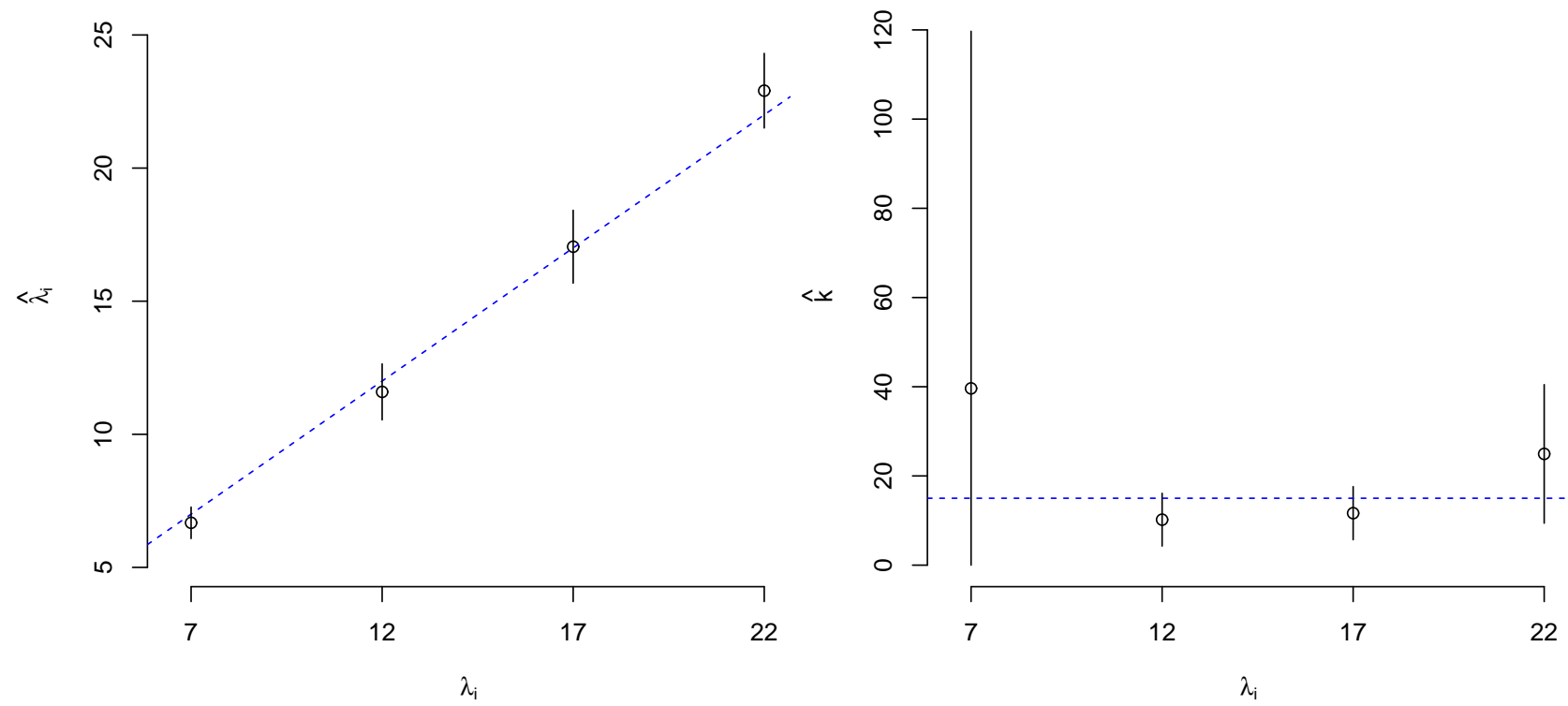

Figura 3.7: Estimativas de máxima verossimilhança $\left(\hat{\lambda_{i}}\right.$ e $\left.\hat{k}\right)$ e intervalos de confiança calculados com base em uma amostra $(n=86)$ de uma Binomial Negativa com média $\lambda_{i}$ e dispersão $k$. 
$\mathrm{i}=1$

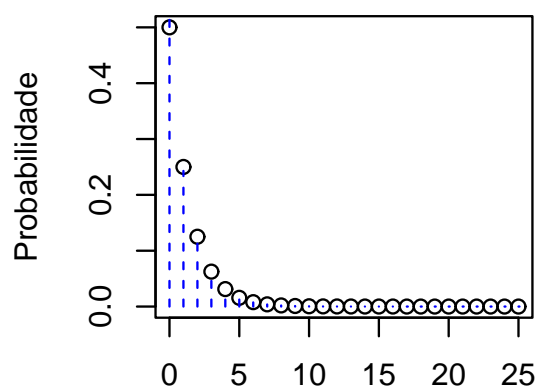

$x$

$\mathbf{i}=\mathbf{3}$

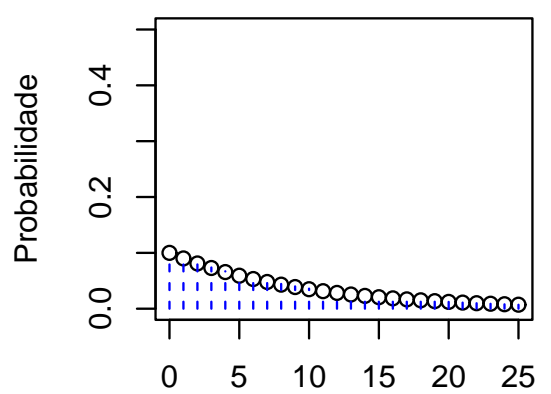

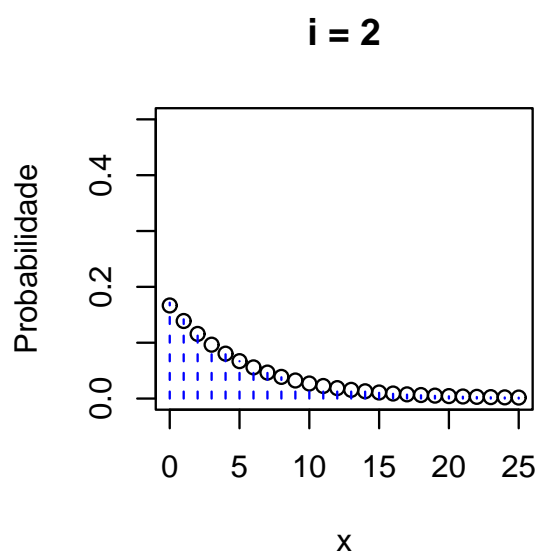

$i=4$

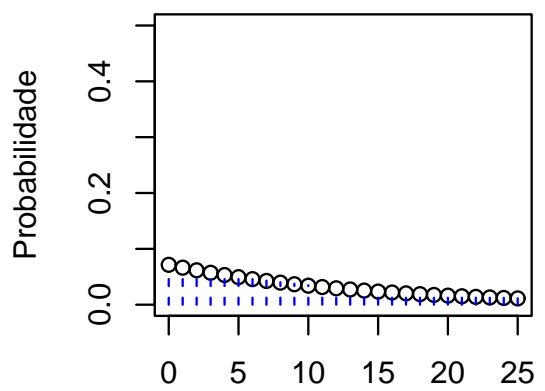

Figura 3.8: Distribuições de probabilidade com paramêtros $\lambda_{i}$ e $k$ (círculos) e distribuições ajustadas (linhas tracejadas) $(\lambda=13, k=15)$.

Tabela 3.12: Estimativas para os parâmetros do modelo (2.14) calculadas com base em uma amostra de tamanho $n=24$ simulada de uma distribuição Binomial Negativa com média $\lambda_{i}$ e $k=350$.

\begin{tabular}{c|ccc|cc}
\hline \hline$i$ & $\lambda_{i}$ & $\widehat{\lambda}_{i}$ & $\mathrm{IC}(95 \%)$ & $\widehat{k}$ & $\mathrm{IC}(95 \%)$ \\
\hline 1 & 18 & 17.250 & {$[15.546 ; 18.954]$} & 332.409 & {$[0 ; 1283.636]$} \\
2 & 19.333 & 18.667 & {$[16.800 ; 20.533]$} & 112.407 & {$[0 ; 558.113]$} \\
3 & 20.667 & 18.417 & {$[16.648 ; 20.185]$} & 301.152 & {$[0 ; 1414.064]$} \\
4 & 22 & 23.583 & {$[21.571 ; 25.596]$} & 322.335 & {$[0 ; 1173.922]$} \\
\hline \hline
\end{tabular}



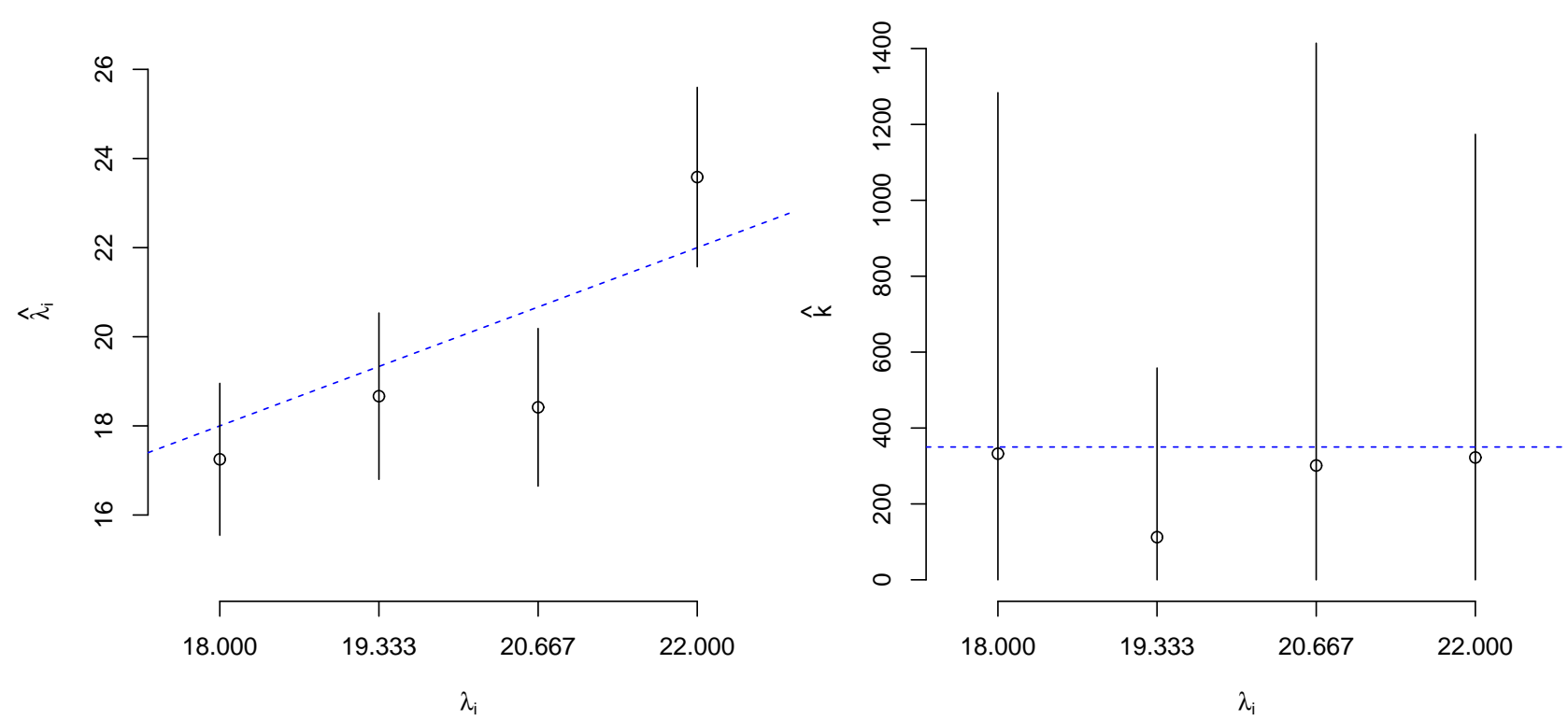

Figura 3.9: Estimativas de máxima verossimilhança $\left(\hat{\lambda_{i}}\right.$ e $\left.\hat{k}\right)$ e intervalos de confiança calculados com base em uma amostra $(n=24)$ de uma Binomial Negativa com média $\lambda_{i}$ e dispersão $k$.
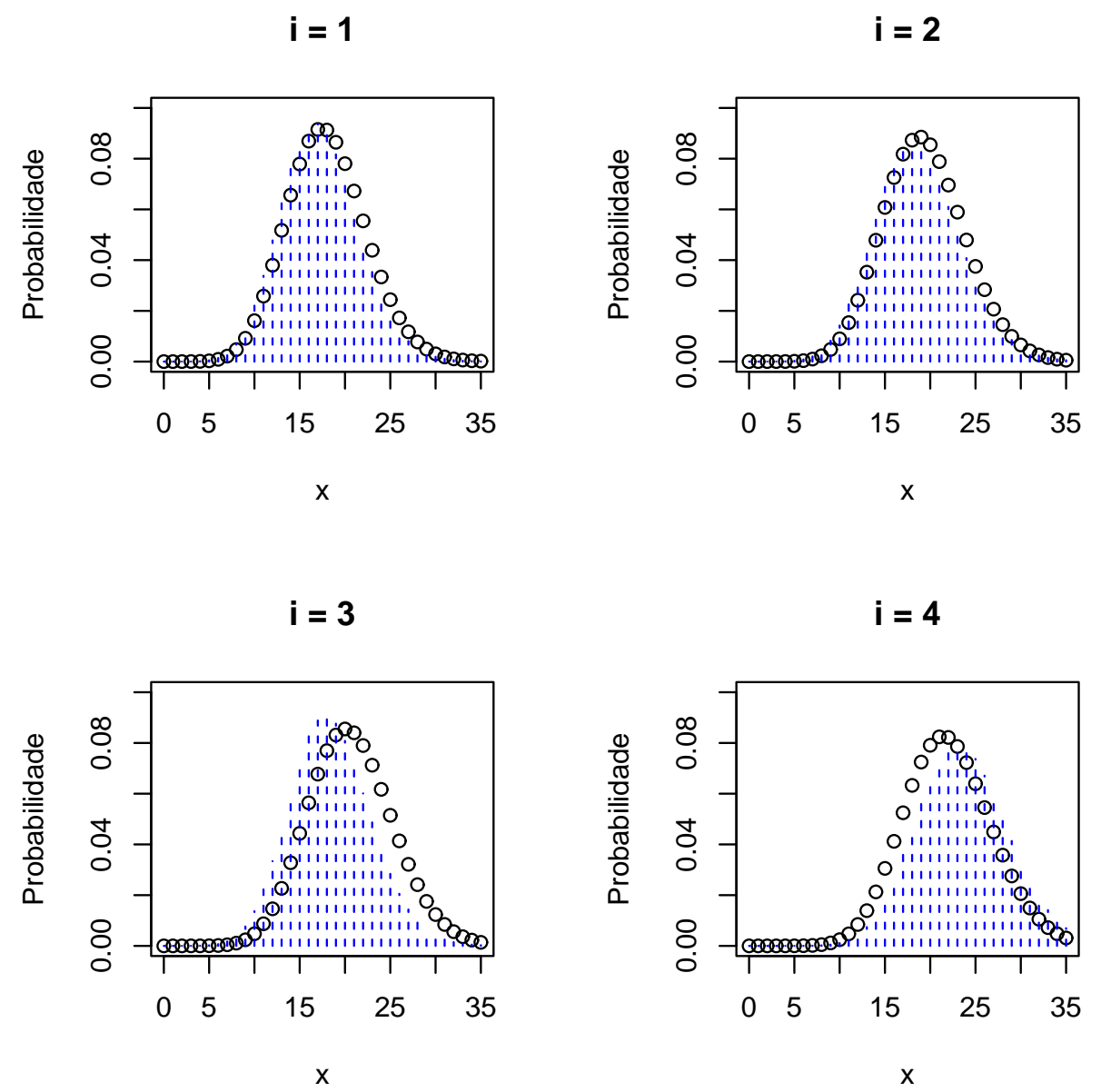

Figura 3.10: Distribuições de probabilidade com paramêtros $\lambda_{i}$ e $k$ (círculos) e distribuições ajustadas (linhas tracejadas) $(\lambda=20, k=350)$. 


\section{Capítulo 4}

\section{Conclusões e sugestões para futuras}

\section{pesquisas}

Neste estudo consideramos o problema de amostragem de água de lastro para avaliação do cumprimento das normas IMO [IMO (2008)]. Propomos métodos estatísticos alternativos que se aproximam mais da realidade do que aqueles apresentados na literatura [Basurko \& Mesbahi (2011); Miller et al. (2011)].

No caso da amostragem estratificada o volume amostral $(v)$ está relacionado com a diferença entre a verdadeira concentração $\lambda$ de organismos e o limite estabelecido pela IMO. Diferenças pequenas requerem volumes amostrais maiores (Tabelas 2.4 e 2.5). Sob o ponto de vista prático, a estratificação de um único tanque pode representar um problema. Realizar a amostragem em cada estrato de maneira que a água de um estrato não se misture com a de outro estrato pode não ser factível. Este modelo é mais aceitável nos casos em que o navio possui dois ou mais tanques com concentrações de organismos possivelmente diferentes e que corresponderiam aos estratos.

A abordagem por meio de processos de Poisson não-homogêneos é uma boa alternativa mas está sujeita ao conhecimento da função que modela o comportamento da concentração de organismos, $\lambda(v)$. Estudos de hidrodinâmica podem ser utilizados para identificação dessa função.

No caso da abordagem por meio de testes de hipóteses usando a distribuição Binomial Negativa o tamanho amostral $(n)$ o tamanho amostral está relacionado ao parâmetro $k$ da distribuição Binomial Negativa que diz respeito à quão variável é a concentração dos organismos no tanque de lastro. Valores pequenos de $k$ requerem tamanhos amostrais maiores, pois tal parâmetro está associado à variabilidade da distribuição da concentração de organismos no tanque, quanto maior o valor de $k$ menor é a variabilidade da distribuição da concentração de organismos. Além disso, o 
tamanho amostral $(n)$ também está relacionado à diferença entre a verdadeira concentração $\lambda$ de organismos e o limite estabelecido pela IMO. Diferenças pequenas requerem tamanhos amostrais maiores (Tabelas 2.18, 2.19 e 2.20). Além disso, neste caso,

A abordagem por meio de estimação é um método alternativo mais adequado pois além de fornecer um procedimento de tomada de decisão permite a avaliação da magnitude da concentração de organismos no tanque de lastro.

As abordagens que usam a distribuição Binomial Negativa (teste de hipóteses e estimação) produzem tamanhos amostrais maiores do que a abordagem que usa a distribuição Poisson, isso é devido a possível heterogeneidade da concentração de organismos que o modelo leva em conta. Neste caso o tamanho amostral está ligado a forma da distribuição da concentração. Um problema prático neste caso é a determinação do valor do parâmetro de dispersão $k$, uma possível solução é obtenção de uma estimativa a priori de informações históricas etc.

Neste trabalho consideramos a norma para organismos $>50 \mu \mathrm{m}$. Embora em alguns casos os volumes amostrais calculados (Ver Tabelas 2.4, 2.5, 2.18, 2.19 e 2.20) envolvam grandes volumes de água de lastro, técnicas de concentração como aquelas descritas em Bierman et al. (2012), podem ser utilizadas para tornar o processo factível. Os resultados obtidos são diretamente transferíveis para avaliação da norma referente a organismos com dimensão entre 10 e $50 \mu m$, bastando para isto substituir a unidade de volume, utilizando $m l$ em vez de $m^{3}$.

Os resultados obtidos aqui também podem ser aplicados a outros problemas de contagem como a distribuição de plantas e animais em um determinado espaço (Ecologia), amostragem de bactéria por unidade de área (Biologia), distribuição do número de lesões focais em virologia (Medicina), distribuição do número de pessoas em uma classe social (Sociologia) etc [Haight (1967)].

Alguns tópicos para pesquisas futuras são:

(a) Aplicar os resultados obtidos a dados reais com o propósito de avaliá-los e aprimorá-los. Adaptar a metodologia desenvolvida aqui para utilização no projeto SAMBA (Sistemas Automáticos de Monitoramento Biológico e Ambiental), que é desenvolvido pelo Instituto Oceanográfico entre outros departamentos da USP e de outras universidades.

(b) Considerar outra distribuição de probabilidade para modelar a concentração de organismos no tanque de lastro. 


\section{Apêndice A}

\section{Demonstrações do Teorema 3.2 e do}

\section{Corolário 3.1}

\section{A.1 Demonstração do Teorema 3.2}

Lema A.1. Sejam $X_{1}, \ldots, X_{n}$ variáveis aleatórias independentes e identicamente distribuídas e $S_{n}=\sum_{i=1}^{n} X_{i} ;$ então

1. $\mathbb{P}\left[S_{n} \geq r\right] \leq \inf _{t>0} e^{-t r}\left[\mu_{X_{1}}(t)\right]^{n} ;$

2. $\mathbb{P}\left[S_{n} \leq r\right] \leq \inf _{t>0} e^{t r}\left[\mu_{X_{1}}(-t)\right]^{n}$,

em que $\mu_{X}(t)$ é a função geradora de momentos de $X$.

Demonstração. Utilizando a desigualdade de Markov temos

$$
\mathbb{P}\left[S_{n} \geq r\right]=\mathbb{P}\left[\exp \left(t S_{n}\right) \geq \exp (t r)\right] \leq \frac{\mathbb{E}\left[\exp \left(t S_{n}\right)\right]}{\exp (t r)}=e^{-t r} \mu_{S_{n}}(t)=e^{-t r}\left[\mu_{X_{1}}(t)\right]^{n}, \quad t>0 .
$$

Logo

$$
\mathbb{P}\left[S_{n} \geq r\right] \leq \inf _{t>0} e^{-t r}\left[\mu_{X_{1}}(t)\right]^{n}
$$

A demonstração para o segundo caso é análoga.

Lema A.2. Seja X uma variável aleatória com distribuição Binomial Negativa com média $\lambda$ e parâmetro de dispersão $k$ (2.14). Então sua função geradora de momentos é $\mu_{X}(t)=\left[1+\frac{\lambda}{k}\left(1-e^{t}\right)\right]^{-k}$.

Lema A.3. Seja $X_{1}, \ldots, X_{n}$ uma amostra aleatória de uma distribuição Binomial Negativa com média $\lambda$ e parâmetro de dispersão $k$ (2.14), $S_{n}=\sum_{i=1}^{n} X_{i}$ e a, uma constante qualquer. Então

1. $\mathbb{P}\left[S_{n} \geq n a\right] \leq \exp \left[n(k+a) \log \left(\frac{k+a}{k+\lambda}\right)-n a \log \left(\frac{a}{\lambda}\right)\right]$ para $\lambda<a ;$

2. $\mathbb{P}\left[S_{n} \leq n a\right] \leq \exp \left[n(k+a) \log \left(\frac{k+a}{k+\lambda}\right)-n a \log \left(\frac{a}{\lambda}\right)\right]$ para $\lambda>a$.

Demonstração. Aplicando os Lemas A.1 e A.2, e fazendo $r=n a$ temos

$$
\mathbb{P}\left[S_{n} \geq n a\right] \leq \inf _{t>0} e^{-t n a}\left[\mu_{X_{1}}(t)\right]^{n}=\inf _{t>0} e^{-t n a}\left[1+\frac{\lambda}{k}\left(1-e^{t}\right)\right]^{-n k}=\inf _{t>0} e^{-n f(t)} k^{n k},
$$


em que $f(t)=a t+k \log \left[k+\lambda\left(1-e^{t}\right)\right]$. Logo

$$
\begin{aligned}
& \text { (i) } f^{\prime}(t)=a-\frac{k \lambda e^{t^{*}}}{k+\lambda\left(1-e^{t^{*}}\right)}=0 \Rightarrow t^{*}=\log \left[\frac{a(k+\lambda)}{\lambda(k+a)}\right]>0, \lambda<a, \\
& \text { (ii) } f^{\prime \prime}(t)=-\frac{k \lambda(k+\lambda) e^{t}}{\left[k+\lambda\left(1-e^{t}\right)\right]^{2}}<0 .
\end{aligned}
$$

De $(i)$ e (ii) concluímos que o valor máximo de $f$ ocorre para $t^{*}=\log \{[a(k+\lambda)] /[\lambda(k+a)]\}$ com $\lambda<a$, o que implica que o ínfimo do limitante superior da desigualdade desejada é atingido também neste ponto; então

$$
\begin{aligned}
\mathbb{P}\left[S_{n} \geq n a\right] \leq e^{-n f\left(t^{*}\right)} k^{n k} & =\exp \left[-n a t^{*}-n k \log \left(k+\lambda\left(1-e^{t^{*}}\right)\right)\right] k^{n k}= \\
& =\exp \left[n(k+a) \log \left(\frac{k+a}{k+\lambda}\right)-n a \log \left(\frac{a}{\lambda}\right)\right] .
\end{aligned}
$$

A demonstração para o segundo caso é análoga.

Em seguida definimos

$$
g(\epsilon, \lambda)=(k+\lambda+\epsilon) \log \left(1+\frac{\epsilon}{k+\lambda}\right)-(\lambda+\epsilon) \log \left(1+\frac{\epsilon}{\lambda}\right) \text { e } \hat{\lambda}=\frac{S_{n}}{n},
$$

para facilitar as demonstrações posteriores

Lema A.4. Para $\lambda>\epsilon>0$, temos $\mathbb{P}[\widehat{\lambda} \leq \lambda-\epsilon] \leq \exp (n g(-\epsilon, \lambda))$ e $g(-\epsilon, \lambda)$ é monotonicamente crescente em relação a $\lambda \in(\epsilon, \infty)$.

Demonstração. Fazendo $a=\lambda-\epsilon$ e aplicando o Lema A.3, para $\lambda>\epsilon$, temos

$$
\begin{aligned}
\mathbb{P}[\widehat{\lambda} \leq \lambda-\epsilon] & =\mathbb{P}\left[S_{n} \leq n a\right] \leq \exp \left[n(k+\lambda-\epsilon) \log \left(1-\frac{\epsilon}{k+\lambda}\right)-n(\lambda-\epsilon) \log \left(1-\frac{\epsilon}{\lambda}\right)\right]= \\
& =\exp (n g(-\epsilon, \lambda)) .
\end{aligned}
$$

Em seguida demonstramos que $g(-\epsilon, \lambda)$ é monotonicamente crescente para $\lambda>\epsilon$. Primeiramente, observemos que

$$
\frac{\partial g(-\epsilon, \lambda)}{\partial \lambda}=\log \left(1-\frac{\epsilon}{k+\lambda}\right)+\frac{\epsilon}{k+\lambda}-\left[\log \left(1-\frac{\epsilon}{\lambda}\right)+\frac{\epsilon}{\lambda}\right] .
$$

Definindo $h(x)=\log (1-x)+x$, temos $h^{\prime}(x)=-x /(1-x)<0$ se $x \in(0,1)$, logo $h(x)$ é uma função decrescente em $x \in(0,1)$; assim temos

$$
\frac{\partial g(-\epsilon, \lambda)}{\partial \lambda}=h\left(\frac{\epsilon}{k+\lambda}\right)-h\left(\frac{\epsilon}{\lambda}\right)>0
$$

implicando que $g(-\epsilon, \lambda)$ é uma função monotonicamente crescente para $\lambda>\epsilon$.

Lema A.5. Para $\epsilon>0$, temos $\mathbb{P}[\widehat{\lambda} \geq \lambda+\epsilon] \leq \exp (n g(\epsilon, \lambda))$ e $g(\epsilon, \lambda)$ é monotonicamente crescente em relação a $\lambda \in(0, \infty)$.

Demonstração. Fazendo $a=\lambda+\epsilon$ e aplicando o Lema A.3, para $\epsilon>0$, temos

$$
\begin{aligned}
\mathbb{P}[\widehat{\lambda} \geq \lambda+\epsilon] & =\mathbb{P}\left[S_{n} \geq n a\right] \leq \exp \left[n(k+\lambda+\epsilon) \log \left(1+\frac{\epsilon}{k+\lambda}\right)-n(\lambda+\epsilon) \log \left(1+\frac{\epsilon}{\lambda}\right)\right]= \\
& =\exp (n g(\epsilon, \lambda)) .
\end{aligned}
$$


Em seguida demonstramos que $g(\epsilon, \lambda)$ é uma função monotonicamente crescente para $\lambda>0$. Primeiramente, observemos que

$$
\frac{\partial g(\epsilon, \lambda)}{\partial \lambda}=\log \left(1+\frac{\epsilon}{k+\lambda}\right)-\frac{\epsilon}{k+\lambda}-\left[\log \left(1+\frac{\epsilon}{\lambda}\right)-\frac{\epsilon}{\lambda}\right] .
$$

Definindo $h(x)=\log (1+x)-x$, temos $h^{\prime}(x)=-x /(1+x)<0$ se $x \in(0, \infty), \log h(x)$ é uma função decrescente em $x \in(0, \infty)$, assim temos que

$$
\frac{\partial g(-\epsilon, \lambda)}{\partial \lambda}=h\left(\frac{\epsilon}{k+\lambda}\right)-h\left(\frac{\epsilon}{\lambda}\right)>0
$$

implicando que $g(\epsilon, \lambda)$ é uma função crescente para $\lambda>0$.

Lema A.6. Para $0<\epsilon<1$, temos $\mathbb{P}[\widehat{\lambda} \leq \lambda(1-\epsilon)] \leq \exp (n g(-\epsilon \lambda, \lambda))$ e $g(-\epsilon \lambda, \lambda)$ é monotonicamente decrescente em relação a $\lambda \in(0, \infty)$.

Demonstração. Fazendo $a=\lambda(1-\epsilon)$ e aplicando o Lema A.3, para $0<\epsilon<1$, temos

$$
\begin{aligned}
\mathbb{P}[\widehat{\lambda} \leq \lambda(1-\epsilon)] & =\mathbb{P}\left[S_{n} \leq n a\right] \leq \exp \left[n(k+\lambda-\lambda \epsilon) \log \left(1-\frac{\lambda \epsilon}{k+\lambda}\right)-n \lambda(1-\epsilon) \log (1-\epsilon)\right]= \\
& =\exp (n g(-\epsilon \lambda, \lambda)) .
\end{aligned}
$$

Em seguida demonstramos que $g(-\epsilon \lambda, \lambda)$ é monotonicamente decrescente para $\lambda>0$. Primeiramente, observemos que

$$
\frac{\partial g(-\epsilon \lambda, \lambda)}{\partial \lambda}=(1-\epsilon) \log \left(1-\frac{\epsilon \lambda}{k+\lambda}\right)+\frac{\epsilon \lambda}{k+\lambda}-[(1-\epsilon) \log (1-\epsilon)+\epsilon]
$$

Definindo $h(x)=(1-\epsilon) \log (1-x \epsilon)+x \epsilon$, temos $h^{\prime}(x)=\epsilon[1-(1-\epsilon) /(1-x \epsilon)]>0$ se $0<x<1$. Logo $h(x)$ é uma função crescente em $0<x<1$, e então

$$
\frac{\partial g(-\epsilon \lambda, \lambda)}{\partial \lambda}=h\left(\frac{\lambda}{k+\lambda}\right)-h(1)<0
$$

implicando que $g(-\epsilon \lambda, \lambda)$ é uma função monotonicamente decrescente para $\lambda>0$.

Lema A.7. Para $\epsilon>0$, temos $\mathbb{P}[\widehat{\lambda} \geq \lambda(1+\epsilon)] \leq \exp (n g(\epsilon \lambda, \lambda))$ e $g(\epsilon \lambda, \lambda)$ é monotonicamente decrescente em relação a $\lambda \in(0, \infty)$.

Demonstração. Fazendo $a=\lambda(1+\epsilon)$ e aplicando o Lema A.3, para $\epsilon>0$, temos

$$
\begin{aligned}
\mathbb{P}[\widehat{\lambda} \geq \lambda(1+\epsilon)] & =\mathbb{P}\left[S_{n} \geq n a\right] \leq \exp \left[n(k+\lambda+\lambda \epsilon) \log \left(1+\frac{\lambda \epsilon}{k+\lambda}\right)-n \lambda(1+\epsilon) \log (1+\epsilon)\right]= \\
& =\exp (n g(\epsilon \lambda, \lambda)) .
\end{aligned}
$$

Em seguida demonstramos que $g(\epsilon \lambda, \lambda)$ é monotonicamente decrescente para $\lambda>0$. Primeiramente, observemos que

$$
\frac{\partial g(\epsilon \lambda, \lambda)}{\partial \lambda}=(1+\epsilon) \log \left(1+\frac{\epsilon \lambda}{k+\lambda}\right)-\frac{\epsilon \lambda}{k+\lambda}-[(1+\epsilon) \log (1+\epsilon)-\epsilon] .
$$

Definindo $h(x)=(1+\epsilon) \log (1+x \epsilon)-x \epsilon$, temos $h^{\prime}(x)=\epsilon[(1+\epsilon) /(1+x \epsilon)-1]>0$ se $0<x<1$, ou seja $h(x)$ é uma função crescente em $0<x<1$. Então

$$
\frac{\partial g(\epsilon \lambda, \lambda)}{\partial \lambda}=h\left(\frac{\lambda}{k+\lambda}\right)-h(1)<0
$$


implicando que $g(\epsilon \lambda, \lambda)$ é uma função monotonicamente decrescente para $\lambda>0$.

Lema A.8. $g(\epsilon, \lambda)>g(-\epsilon, \lambda)$ para $\lambda>\epsilon$.

Demonstração. Primeiramente notemos que

$\frac{\partial g(\epsilon, \lambda)}{\partial \epsilon}=\log \left(1+\frac{\epsilon}{k+\lambda}\right)-\log \left(1+\frac{\epsilon}{\lambda}\right) \quad$ e $\quad \frac{\partial g(-\epsilon, \lambda)}{\partial \epsilon}=-\log \left(1-\frac{\epsilon}{k+\lambda}\right)+\log \left(1-\frac{\epsilon}{\lambda}\right)$.

Logo

$$
\frac{\partial[g(\epsilon, \lambda)-g(-\epsilon, \lambda)]}{\partial \epsilon}=\log \left(1+\frac{\epsilon}{k+\lambda}\right)+\log \left(1-\frac{\epsilon}{k+\lambda}\right)-\left[\log \left(1+\frac{\epsilon}{\lambda}\right)+\log \left(1-\frac{\epsilon}{\lambda}\right)\right] .
$$

Definindo $h(x)=\log (1+x)+\log (1-x)=\log \left(1-x^{2}\right)$, temos $h^{\prime}(x)=-2 x /(1-x)<0$ se $x \in(0,1)$.

Então $h(x)$ é uma função decrescente em $x \in(0,1)$, e

$$
\frac{\partial[g(\epsilon, \lambda)-g(-\epsilon, \lambda)]}{\partial \epsilon}=h\left(\frac{\epsilon}{k+\lambda}\right)-h\left(\frac{\epsilon}{\lambda}\right)>0,
$$

implicando que $g(\epsilon, \lambda)>g(-\epsilon, \lambda)$ para $\lambda>\epsilon$.

Para demonstrar o Teorema 3.2 é suficiente mostrar que $\mathbb{P}\left[|\widehat{\lambda}-\lambda| \geq \epsilon_{a}\right.$ e $\left.|\widehat{\lambda}-\lambda| \geq \epsilon_{r} \lambda\right]<\delta$ para $n$ satisfazendo (3.3), e considerar os seguintes casos:

- Caso 1: $0<\lambda<\epsilon_{a}$;

- Caso 2: $\lambda=\epsilon_{a}$;

- Caso 3: $\epsilon_{a}<\lambda \leq \epsilon_{a} / \epsilon_{r}$;

- Caso 4: $\lambda>\epsilon_{a} / \epsilon_{r}$.

Caso 1: Aplicando o Lema A.5 temos que

$$
\begin{aligned}
\mathbb{P}\left[|\widehat{\lambda}-\lambda| \geq \epsilon_{a} \text { e }|\widehat{\lambda}-\lambda| \geq \epsilon_{r} \lambda\right] & =\mathbb{P}\left[|\widehat{\lambda}-\lambda| \geq \epsilon_{a}\right]=\mathbb{P}\left[\widehat{\lambda} \leq \lambda-\epsilon_{a}\right]+\mathbb{P}\left[\widehat{\lambda} \geq \lambda+\epsilon_{a}\right]= \\
& =\mathbb{P}\left[\widehat{\lambda} \geq \lambda+\epsilon_{a}\right] \leq \exp \left(n g\left(\epsilon_{a}, \lambda\right)\right) \leq \exp \left(n g\left(\epsilon_{a}, \epsilon_{a}\right)\right) \leq \\
& \leq \exp \left(n g\left(\epsilon_{a}, \epsilon_{a} / \epsilon_{r}\right)\right)<\delta / 2 .
\end{aligned}
$$

Caso 2: Aplicando o Lema A.5 temos que

$$
\begin{aligned}
\mathbb{P}\left[|\widehat{\lambda}-\lambda| \geq \epsilon_{a} \text { e }|\widehat{\lambda}-\lambda| \geq \epsilon_{r} \lambda\right] & =\mathbb{P}\left[|\widehat{\lambda}-\lambda| \geq \epsilon_{a}\right]=\mathbb{P}\left[\widehat{\lambda} \leq \lambda-\epsilon_{a}\right]+\mathbb{P}\left[\widehat{\lambda} \geq \lambda+\epsilon_{a}\right]= \\
& =\mathbb{P}[\widehat{\lambda}=0]+\mathbb{P}\left[\widehat{\lambda} \geq \lambda+\epsilon_{a}\right]=\mathbb{P}\left[X_{i}=0, \forall i\right]+\mathbb{P}\left[\widehat{\lambda} \geq \lambda+\epsilon_{a}\right]= \\
& =\left[\mathbb{P}\left[X_{1}=0\right]\right]^{n}+\mathbb{P}\left[\widehat{\lambda} \geq \lambda+\epsilon_{a}\right]= \\
& =\exp \left[n k \log \left(\frac{k}{k+\epsilon_{a}}\right)\right]+\mathbb{P}\left[\widehat{\lambda} \geq \lambda+\epsilon_{a}\right] \leq 2 \exp \left(n g\left(\epsilon_{a}, \epsilon_{a}\right)\right) \leq \\
& \leq 2 \exp \left(n g\left(\epsilon_{a}, \epsilon_{a} / \epsilon_{r}\right)\right)<\delta .
\end{aligned}
$$

Caso 3: Aplicando os Lemas A.4, A.5 e A.8 temos que

$$
\begin{aligned}
\mathbb{P}\left[|\widehat{\lambda}-\lambda| \geq \epsilon_{a} \text { e }|\widehat{\lambda}-\lambda| \geq \epsilon_{r} \lambda\right] & =\mathbb{P}\left[\widehat{\lambda} \leq \lambda-\epsilon_{a}\right]+\mathbb{P}\left[\widehat{\lambda} \geq \lambda+\epsilon_{a}\right] \leq \\
& \leq \exp \left(n g\left(-\epsilon_{a}, \lambda\right)\right)+\exp \left(n g\left(\epsilon_{a}, \lambda\right)\right) \leq \\
& \leq \exp \left(n g\left(-\epsilon_{a}, \epsilon_{a} / \epsilon_{r}\right)\right)+\exp \left(n g\left(\epsilon_{a}, \epsilon_{a} / \epsilon_{r}\right)\right) \leq \\
& \leq 2 \exp \left(n g\left(\epsilon_{a}, \epsilon_{a} / \epsilon_{r}\right)\right)<\delta .
\end{aligned}
$$


Caso 4: Aplicando os Lemas A.6, A.7 e A.8 temos que

$$
\begin{aligned}
\mathbb{P}\left[|\widehat{\lambda}-\lambda| \geq \epsilon_{a} \text { e }|\widehat{\lambda}-\lambda| \geq \epsilon_{r} \lambda\right] & =\mathbb{P}\left[|\widehat{\lambda}-\lambda| \geq \epsilon_{r} \lambda\right]=\mathbb{P}\left[\widehat{\lambda} \leq \lambda\left(1-\epsilon_{r}\right)\right]+\mathbb{P}\left[\widehat{\lambda} \geq \lambda\left(1+\epsilon_{r}\right)\right] \leq \\
& \leq \exp \left(n g\left(-\epsilon_{r} \lambda, \lambda\right)\right)+\exp \left(n g\left(\epsilon_{r} \lambda, \lambda\right)\right) \leq \\
& \leq \exp \left(n g\left(-\epsilon_{a}, \epsilon_{a} / \epsilon_{r}\right)\right)+\exp \left(n g\left(\epsilon_{a}, \epsilon_{a} / \epsilon_{r}\right)\right) \leq \\
& \leq 2 \exp \left(n g\left(\epsilon_{a}, \epsilon_{a} / \epsilon_{r}\right)\right)<\delta,
\end{aligned}
$$

o que completa a demonstração.

\section{A.2 Demonstração do Corolário 3.1}

Primeiramente note que somente a segunda expressão do denominador de (3.3) depende de $k$; então

$$
\begin{aligned}
& \lim _{k \rightarrow \infty}\left[\left(\frac{\epsilon_{r}}{\epsilon_{a}} k+1+\epsilon_{r}\right) \log \left(\frac{k \epsilon_{r}+\epsilon_{a}+\epsilon_{a} \epsilon_{r}}{k \epsilon_{r}+\epsilon_{a}}\right)\right]=\frac{1}{\epsilon_{a}} \lim _{k \rightarrow \infty}\left[\epsilon_{a} \epsilon_{r} \log \left(1+\frac{\epsilon_{a} \epsilon_{r}}{k \epsilon_{r}+\epsilon_{a}}\right)\right]+ \\
& +\frac{1}{\epsilon_{a}} \lim _{k \rightarrow \infty}\left[\log \left(1+\frac{\epsilon_{a} \epsilon_{r}}{k \epsilon_{r}+\epsilon_{a}}\right)^{k \epsilon_{r}+\epsilon_{a}}\right]=\frac{1}{\epsilon_{a}}\left(0+\log e^{\epsilon_{a} \epsilon_{r}}\right)=\frac{1}{\epsilon_{a}} \epsilon_{a} \epsilon_{r}=\epsilon_{r},
\end{aligned}
$$

completando a demonstração. 
APÊNDICE A 


\section{Apêndice B}

\section{Demonstração Teorema 3.3}

Os Lemas B.1, B.2, B.3, B.4 e B.7 são contribuições de Chen (2011) e foram reproduzidos nesta dissertação para facilitar a compreensão do leitor. Os Lemas B.5 e B.6 são extensões de resultados de Chen (2011) para o caso da Binomial Negativa.

Definamos a probabilidade de cobertura como

$$
C(\lambda)=\mathbb{P}[|\bar{X}-\lambda|<\epsilon]=\mathbb{P}\left[g(\lambda) \leq \sum_{i=1}^{n} X_{i} \leq h(\lambda)\right],
$$

em que $X_{1}, X_{2}, \ldots, X_{n}$ é uma amostra aleatória com distribuição Binomial Negativa com média $\lambda$ e parâmetro de dispersão $k, \bar{X}=n^{-1} \sum_{i=1}^{n} X_{i}, g(\lambda)=\lfloor n(\lambda-\epsilon)\rfloor+1$ e $h(\lambda)=\lceil n(\lambda+\epsilon)\rceil-1$. Consideremos a função de probabilidade da distribuição Binomial Negativa definida em (2.14) denotada por $B N(\lambda, x, k)$. Também definamos

$$
S(\lambda, g, h, k)=\sum_{x=g}^{h} B N(\lambda, x, k) .
$$

Lema B.1 (Chen, 2011). Seja $\lambda_{\ell}=\ell / n-\epsilon$ em que $\ell \in \mathbb{Z}$. Então $h(\lambda)=h\left(\lambda_{\ell+1}\right)=\ell$ para todo $\lambda \in\left(\lambda_{\ell}, \lambda_{\ell+1}\right)$.

Demonstração. Para $\lambda \in\left(\lambda_{\ell}, \lambda_{\ell+1}\right)$, temos $0<n\left(\lambda-\lambda_{\ell}\right)<1 \mathrm{e}$

$$
\begin{aligned}
h(\lambda) & =\lceil n(\lambda+\epsilon)\rceil-1=\left\lceil n\left(\lambda_{\ell}+\epsilon+\lambda-\lambda_{\ell}\right)\right\rceil-1=\left\lceil n\left(\frac{\ell}{n}-\epsilon+\epsilon+\lambda-\lambda_{\ell}\right)\right\rceil-1= \\
& =\ell-1+\left\lceil n\left(\lambda-\lambda_{\ell}\right)\right\rceil=\ell=\left\lceil n\left(\frac{\ell+1}{n}-\epsilon+\epsilon\right)\right\rceil-1=h\left(\lambda_{\ell+1}\right) .
\end{aligned}
$$

Lema B.2 (Chen, 2011). Seja $\lambda_{\ell}=\ell / n+\epsilon$ em que $\ell \in \mathbb{Z}$. Então, $g(\lambda)=g\left(\lambda_{\ell}\right)=\ell+1$ para todo $\lambda \in\left(\lambda_{\ell}, \lambda_{\ell+1}\right)$.

Demonstração. Para $\lambda \in\left(\lambda_{\ell}, \lambda_{\ell+1}\right)$, temos $-1<n\left(\lambda-\lambda_{\ell+1}\right)<0$ e

$$
\begin{aligned}
g(\lambda) & =\lfloor n(\lambda-\epsilon)\rfloor+1=\left\lfloor n\left(\lambda_{\ell+1}-\epsilon+\lambda-\lambda_{\ell+1}\right)\right\rfloor+1= \\
& =\left\lfloor n\left(\frac{\ell+1}{n}+\epsilon-\epsilon\right)\right\rfloor+\left\lfloor n\left(\lambda-\lambda_{\ell+1}\right)\right\rfloor+1= \\
& =\left\lfloor n\left(\frac{\ell+1}{n}+\epsilon-\epsilon\right)\right\rfloor-1+1=\ell+1=\left\lfloor n\left(\frac{\ell}{n}+\epsilon-\epsilon\right)\right\rfloor+1=g\left(\lambda_{\ell}\right) .
\end{aligned}
$$


Lema B.3 (Chen, 2011). Sejam $\alpha<\beta$ dois elementos consecutivos do arranjo ascendente de todos os elementos distintos de $\{a, b\} \cup\{\ell / n+\epsilon \in(a, b): \ell \in \mathbb{Z}\} \cup\{\ell / n-\epsilon \in(a, b): \ell \in \mathbb{Z}\}$. Então, $g(\mu)$ e $h(\mu)$ são constantes para qualquer $\mu \in(\alpha, \beta)$.

Demonstração. Uma vez que $\alpha$ e $\beta$ são dois elementos consecutivos do arranjo ascendente de todos os elementos distintos do conjunto, não há inteiro $\ell$ tal que $\alpha<\ell / n+\epsilon<\beta$ or $\alpha<\ell / n-\epsilon<\beta$. Logo existem dois inteiros $\ell$ e $\ell^{\prime}$ tais que $(\alpha, \beta) \subseteq(\ell / n+\epsilon,(\ell+1) / n+\epsilon)$ e $(\alpha, \beta) \subseteq\left(\ell^{\prime} / n-\epsilon,\left(\ell^{\prime}+1\right) / n-\epsilon\right)$. Aplicando os Lemas B.1 e B.2, temos $g(\mu)=g(\ell / n+\epsilon)$ e $h(\mu)=h\left(\left(\ell^{\prime}+1\right) / n-\epsilon\right)$ para todo $\mu \in(\alpha, \beta)$.

Lema B.4 (Chen, 2011). Para todo $\lambda \in(0, \infty), \lim _{\eta \rightarrow 0} C(\lambda+\eta) \geq C(\lambda)$ e $\lim _{\eta \rightarrow 0} C(\lambda-\eta) \geq C(\lambda)$.

Demonstração. Observemos que $h(\lambda+\eta) \geq h(\lambda)$ para todo $\eta>0$ e que

$$
\begin{aligned}
g(\lambda+\eta) & =\lfloor n(\lambda+\eta-\epsilon)\rfloor+1=\lfloor n(\lambda-\epsilon)\rfloor+1+\lfloor n(\lambda-\epsilon)-\lfloor n(\lambda-\epsilon)\rfloor+n \eta\rfloor= \\
& =\lfloor n(\lambda-\epsilon)\rfloor+1=g(\lambda)
\end{aligned}
$$

para $0<\eta<\{1+\lfloor n(\lambda-\epsilon)\rfloor-n(\lambda-\epsilon)\} / n$. Então

$$
S(n(\lambda+\eta), g(\lambda+\eta), h(\lambda+\eta), n k) \geq S(n(\lambda+\eta), g(\lambda), h(\lambda), n k)
$$

para $0<\eta<\{1+\lfloor n(\lambda-\epsilon)\rfloor-n(\lambda-\epsilon)\} / n$. Uma vez que

$$
h(\lambda+\eta)=\lceil n(\lambda+\eta+\epsilon)\rceil-1=\lceil n(\lambda+\epsilon)\rceil-1+\lceil n(\lambda+\epsilon)-\lceil n(\lambda+\epsilon)\rceil+n \eta\rceil,
$$

temos

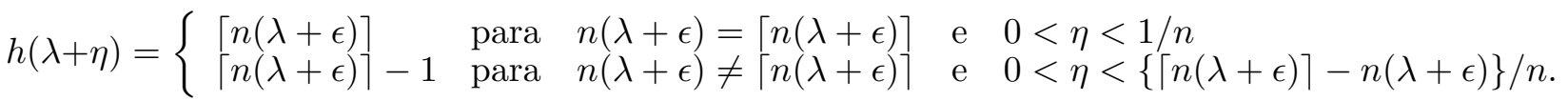

Logo $g(\lambda+\eta)$ e $h(\lambda+\eta)$ são independente de $\eta$ se $\eta>0$ é pequeno o bastante. $S(n, g, h, \lambda+\eta)$ é contínua em relação a $\eta$ para $g$ e $h$ fixados, o $\lim _{\eta \rightarrow 0} S(n(\lambda+\eta), g(\lambda+\eta), h(\lambda+\eta), n k)$ existe. Então

$$
\begin{aligned}
\lim _{\eta \rightarrow 0} C(\lambda+\eta) & =\lim _{\eta \rightarrow 0} S(n(\lambda+\eta), g(\lambda+\eta), h(\lambda+\eta), n k) \geq \lim _{\eta \rightarrow 0} S(n(\lambda+\eta), g(\lambda), h(\lambda), n k)= \\
& =S(n \lambda, g(\lambda), h(\lambda), n k)=C(\lambda)
\end{aligned}
$$

em que a desigualdade segue de (B.2). Observemos que $g(\lambda-\eta) \leq g(\lambda)$ para todo $\eta>0$ e que

$$
\begin{aligned}
h(\lambda-\eta) & =\lceil n(\lambda-\eta+\epsilon)\rceil-1=\lceil n(\lambda+\epsilon)\rceil-1+\lceil n(\lambda+\epsilon)-\lceil n(\lambda+\epsilon)\rceil-n \eta\rceil= \\
& =\lceil n(\lambda+\epsilon)\rceil-1=h(\lambda)
\end{aligned}
$$

para $0<\eta<\{1+n(\lambda+\epsilon)-\lceil n(\lambda+\epsilon)\rceil\} / n$. Então

$$
S(n(\lambda-\eta), g(\lambda-\eta), h(\lambda-\eta), n k) \geq S(n(\lambda-\eta), g(\lambda), h(\lambda), n k)
$$

para $0<\eta<\{1+n(\lambda+\epsilon)-\lceil n(\lambda+\epsilon)\rceil\} / n$. Uma vez que

$$
g(\lambda-\eta)=\lfloor n(\lambda-\eta-\epsilon)\rfloor+1=\lfloor n(\lambda-\epsilon)\rfloor+1+\lfloor n(\lambda-\epsilon)-\lfloor n(\lambda-\epsilon)\rfloor-n \eta\rfloor,
$$

temos

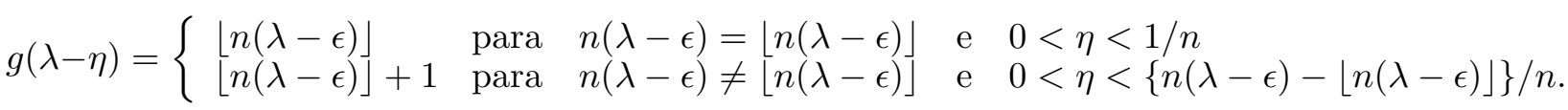

Logo $g(\lambda-\eta)$ e $h(\lambda-\eta)$ são independentes de $\eta$ se $\eta>0$ é pequeno o bastante. Uma vez que $S(n, g, h, \lambda-\eta)$ é contínua com relação a $\eta$ para $g$ e $h$ fixos, o $\lim _{\eta \rightarrow 0} S(n(\lambda-\eta), g(\lambda-\eta), h(\lambda-\eta), n k)$ 
existe. Então

$$
\begin{aligned}
\lim _{\eta \rightarrow 0} C(\lambda-\eta) & =\lim _{\eta \rightarrow 0} S(n(\lambda-\eta), g(\lambda-\eta), h(\lambda-\eta), n k) \geq \lim _{\eta \rightarrow 0} S(n(\lambda-\eta), g(\lambda), h(\lambda), n k)= \\
& =S(n \lambda, g(\lambda), h(\lambda), n k)=C(\lambda)
\end{aligned}
$$

em que a desigualdade segue de (B.3).

Lema B.5. Sejam $h, g, k>0, g \leq h$ e $\Gamma$ a função gama. Então

$$
\frac{\Gamma(n k+h+1) \Gamma(g)}{\Gamma(n k+g) \Gamma(h+1)}>1
$$

Demonstração. Primeiramente, notemos que

$$
\frac{\Gamma(n k+h+1) \Gamma(g)}{\Gamma(n k+g) \Gamma(h+1)}=\left(\frac{n k}{h}+1\right) \frac{\Gamma(n k+h) \Gamma(g)}{\Gamma(h) \Gamma(n k+g)} .
$$

Então é suficiente mostrar que $\frac{\Gamma(n k+h) \Gamma(g)}{\Gamma(h) \Gamma(n k+g)}>1$. Suponhamos que

$$
\frac{\Gamma(n k+h) \Gamma(g)}{\Gamma(h) \Gamma(n k+g)}<1
$$

que implica

$$
\frac{\Gamma(h) \Gamma(n k)}{\Gamma(h+n k)}>\frac{\Gamma(g) \Gamma(n k)}{\Gamma(g+n k)} .
$$

Logo $B(h, n k)>B(g, n k)$, em que $B(x, y)=\int_{0}^{1} t^{x-1}(1-t)^{y-1} d t$ é a função beta. Mas notemos que como

$$
B(x, y)=\frac{\Gamma(x) \Gamma(y)}{\Gamma(x+y)}=\exp [\log \Gamma(x)+\log \Gamma(y)-\log \Gamma(x+y)]
$$

obtemos

$$
\frac{\partial}{\partial x} B(x, y)=B(x, y)[\psi(x)-\psi(x+y)]
$$

em que $\psi($.$) é a função digama. Como \psi($.$) é uma função crescente [Beals \& Wong (2012)], \psi(x)-$ $\psi(x+y)<0$ e assim $B(x, y)$ é decrescente em $x$ para $y$ fixo. Logo $B(h, n k)>B(g, n k)$ não pode ser verdade pois $g \leq h$.

Lema B.6. Sejam $0<u<v<\infty$ e $g \leq h$. Então

$$
\min _{\lambda \in[u, v]} S(n \lambda, g, h, n k)=\min \{S(n u, g, h, n k), S(n v, g, h, n k)\} .
$$

Demonstração. Primeiramente, notemos que

$$
\begin{aligned}
\frac{\partial}{\partial \lambda} B N(n \lambda, x, n k) & =\frac{\partial}{\partial \lambda}[\exp (\log B N(n \lambda, x, n k))]=\exp [\log B N(n \lambda, x, n k)] \frac{\partial}{\partial \lambda} \log B N(n \lambda, x, n k)= \\
& =B N(n \lambda, x, n k)\left(\frac{x}{\lambda}-\frac{x+n k}{\lambda+k}\right)
\end{aligned}
$$


também notemos que

$$
\begin{aligned}
\frac{x+n k}{\lambda+k} B N(n \lambda, x, n k) & =\frac{x+n k}{\lambda+k} \frac{\Gamma(n k+x)}{\Gamma(x+1) \Gamma(n k)}\left(\frac{\lambda}{\lambda+k}\right)^{x}\left(\frac{k}{\lambda+k}\right)^{n k}= \\
& =\frac{x+1}{\lambda} \frac{\Gamma(n k+x+1)}{\Gamma(x+2) \Gamma(n k)}\left(\frac{\lambda}{\lambda+k}\right)^{x+1}\left(\frac{k}{\lambda+k}\right)^{n k}= \\
& =\frac{x+1}{\lambda} B N(n \lambda, x+1, n k) .
\end{aligned}
$$

Logo, de (B.4) e (B.5) temos

$$
\frac{\partial}{\partial \lambda} B N(n \lambda, x, n k)=\frac{x}{\lambda} B N(n \lambda, x, n k)-\frac{x+1}{\lambda} B N(n \lambda, x+1, n k) .
$$

Então,

$$
\begin{aligned}
\frac{\partial}{\partial \lambda} S(n \lambda, 0, l, n k) & =\sum_{x=0}^{l} \frac{\partial}{\partial \lambda} B N(n \lambda, x, n k)=\sum_{y=0}^{l}\left[\frac{x}{\lambda} B N(n \lambda, x, n k)-\frac{x+1}{\lambda} B N(n \lambda, x+1, n k)\right]= \\
& =\sum_{x=1}^{l} \frac{x}{\lambda} B N(n \lambda, x, n k)-\sum_{x=0}^{l} \frac{x+1}{\lambda} B N(n \lambda, x+1, n k)= \\
& =\sum_{x=1}^{l} \frac{x}{\lambda} B N(n \lambda, x, n k)-\sum_{x=1}^{l+1} \frac{x}{\lambda} B N(n \lambda, x, n k)=-\frac{l+1}{\lambda} B N(n \lambda, l+1, n k) .(\text { B.6 })
\end{aligned}
$$

É necessário considerar os seguintes casos.

(1) $g \leq h<0$. Neste caso, $S(n \lambda, g, h, n k)=0$ para qualquer $\lambda \in[u, v]$ e claramente o Lema é verdade.

(2) $g \leq 0 \leq h$. Neste caso, $S(n \lambda, g, h, n k)=S(n \lambda, 0, h, n k)$, que é uma função decrescente de acordo com (B.6) e claramente o Lema é verdade.

(3) $0<g \leq h$. Através de (B.6) temos

$$
\begin{aligned}
\frac{\partial}{\partial \lambda} C(\lambda) & =\frac{\partial}{\partial \lambda} S(n \lambda, 0, h, n k)-\frac{\partial}{\partial \lambda} S(n \lambda, 0, g-1, n k)= \\
& =-\frac{h+1}{\lambda} B N(n \lambda, h+1, n k)+\frac{g}{\lambda} B N(n \lambda, g, n k)= \\
& =\frac{g}{\lambda} \frac{\Gamma(n k+g)}{\Gamma(g+1) \Gamma(n k)}\left(\frac{\lambda}{\lambda+k}\right)^{g}\left(\frac{k}{\lambda+k}\right)^{n k}-\frac{h+1}{\lambda} \frac{\Gamma(n k+h+1)}{\Gamma(h+2) \Gamma(n k)}\left(\frac{\lambda}{\lambda+k}\right)^{h+1}\left(\frac{k}{\lambda+k}\right)^{n k}= \\
& =\frac{k^{n k}}{\Gamma(n k)(\lambda+k)^{n k}}\left[\frac{\Gamma(n k+g) \lambda^{g-1}}{\Gamma(g)(\lambda+k)^{g}}-\frac{\Gamma(n k+h+1) \lambda^{h}}{\Gamma(h+1)(\lambda+k)^{h+1}}\right]= \\
& =\frac{\Gamma(n k+h+1)}{\Gamma(n k) \Gamma(h+1)} \frac{\lambda^{g-1}}{(\lambda+k)^{g}}\left(\frac{k}{\lambda+k}\right)^{n k}\left[\frac{\Gamma(h+1) \Gamma(n k+g)}{\Gamma(n k+h+1) \Gamma(g)}-\left(\frac{\lambda}{\lambda+k}\right)^{h-g+1}\right] .
\end{aligned}
$$

Consequentemente,

$$
\frac{\partial C(\lambda)}{\partial \lambda}>0 \quad \text { se } \quad \lambda<\xi=k\left\{\left[\frac{\Gamma(n k+h+1) \Gamma(g)}{\Gamma(n k+g) \Gamma(h+1)}\right]^{1 /(h-g+1)}-1\right\}^{-1} .
$$

Pelo Lema B.5 $\xi>0$, então a função $C(\lambda)$ pode ser monotonicamente crescente (decrescente) no intervalo $[u, v]$, ou a função $C(\lambda)$ cresce até um determinado ponto do intervalo $[u, v] \mathrm{e}$ depois decresce, o que completa a demonstração. 
Lema B.7 (Chen, 2011). Sejam $\alpha<\beta$ dois elementos consecutivos do arranjo ascendente de todos os elementos distintos de $\{a, b\} \cup\{\ell / n+\epsilon \in(a, b): \ell \in \mathbb{Z}\} \cup\{\ell / n-\epsilon \in(a, b): \ell \in \mathbb{Z}\}$. Então, $C(\lambda) \geq \min \{C(\alpha), C(\beta)\}$ para todo $\lambda \in(\alpha, \beta)$.

Demonstração. Pelo Lema B.3, $g(\lambda)$ e $h(\lambda)$ são constantes para qualquer $\lambda \in(\alpha, \beta)$. Por isso, omitimos o argumento e escrevemos $g(\lambda)=g, h(\lambda)=h$ e $C(\lambda)=S(\lambda, g, h, k)$. Para $\lambda \in(\alpha, \beta)$, definamos o intervalo $[\alpha+\eta, \beta-\eta] \operatorname{com} 0<\eta<\min (\lambda-\alpha, \beta-\lambda,(\beta-\alpha) / 2)$. Então, $\lambda \in[\alpha+\eta, \beta-\eta]$. Pelo Lema B.6,

$$
C(\lambda) \geq \min _{\omega \in[\alpha+\eta, \beta-\eta]} C(\omega)=\min \{C(\alpha+\eta), C(\beta-\eta)\}
$$

para $0<\eta<\min \{\lambda-\alpha, \beta-\lambda,(\beta-\alpha) / 2\}$. Pelo Lema B.4, $\lim _{\eta \rightarrow 0} C(\alpha+\eta)$ e $\lim _{\eta \rightarrow 0} C(\beta-\eta)$ existem e são limitados abaixo por $C(\alpha)$ e $C(\beta)$, respectivamente. Logo

$$
C(\lambda) \geq \lim _{\eta \rightarrow 0} \min \{C(\alpha+\eta), C(\beta-\eta)\}=\min \left\{\lim _{\eta \rightarrow 0} C(\alpha+\eta), \lim _{\eta \rightarrow 0} C(\beta-\eta)\right\} \geq \min \{C(\alpha), C(\beta)\},
$$

para qualquer $\lambda \in(\alpha, \beta)$.

A afirmação sobre o mínimo da probabilidade de cobertura segue imediatamente do Lema B.7. O número de elementos do conjunto discreto descrito no Teorema 3.3 pode ser calculado como indicado em Chen (2011). Como

$$
a<\ell / n-\epsilon<b \Leftrightarrow 1+\lfloor n(a+\epsilon)\rfloor \leq \ell \leq\lceil n(b+\epsilon)\rceil,
$$

o número de elementos em $\{\ell / n-\epsilon \in(a, b): \ell \in \mathbb{Z}\}$ é

$$
\lceil n(b+\epsilon)\rceil-\lfloor n(a+\epsilon)\rfloor-1<n(b+\epsilon)+1-[n(a+\epsilon)-1]-1=n(b-a)+1 .
$$

Por outro lado,

$$
a<\ell / n+\epsilon<b \Leftrightarrow 1+\lfloor n(a-\epsilon)\rfloor \leq \ell \leq\lceil n(b-\epsilon)\rceil-1,
$$

e o número de elementos em $\{\ell / n+\epsilon \in(a, b): \ell \in \mathbb{Z}\}$ é

$$
\lceil n(b-\epsilon)\rceil-\lfloor n(a-\epsilon)\rfloor-1<n(b-\epsilon)+1-[n(a-\epsilon)-1]-1=n(b-a)+1 .
$$

Consequentemente, o número total de elementos no conjunto $\{a, b\} \cup\{\ell / n+\epsilon \in(a, b): \ell \in$ $\mathbb{Z}\} \cup\{\ell / n-\epsilon \in(a, b): \ell \in \mathbb{Z}\}$ é menor que $2 n(b-a)+4$, o que completa a demonstração. 
APÊNDICE B 


\section{Apêndice $\mathrm{C}$}

\section{Demonstrações dos Teoremas 3.4 e 3.5}

\section{C.1 Demonstração do Teorema 3.4}

Os Lemas C.1, C.2, C.3, C.4 e C.5 são contribuições de Chen (2011) e foram reproduzidos nesta dissertação para facilitar a compreensão do leitor.

Definamos a probabilidade de cobertura como

$$
C(\lambda)=\mathbb{P}[|\bar{X}-\lambda|<\lambda \epsilon]=\mathbb{P}\left[g(\lambda) \leq \sum_{i=1}^{n} X_{i} \leq h(\lambda)\right]
$$

em que $X_{1}, X_{2}, \ldots, X_{n}$ é uma amostra aleatória com distribuição Binomial Negativa com média $\lambda$ e parâmetro de dispersão $k, \bar{X}=n^{-1} \sum_{i=1}^{n} X_{i}, g(\lambda)=\lfloor n \lambda(1-\epsilon)\rfloor+1$ e $h(\lambda)=\lceil n \lambda(1+\epsilon)\rceil-1$. Seja a função de probabilidade da distribuição Binomial Negativa definida em (2.14) denotada por $B N(\lambda, x, k)$. Também definamos

$$
S(\lambda, g, h, k)=\sum_{x=g}^{h} B N(\lambda, x, k)
$$

Lema C.1 (Chen, 2011). Seja $\lambda_{\ell}=\ell /(n(1+\epsilon))$ em que $\ell \in \mathbb{Z}$. Então, $h(\lambda)=h\left(\lambda_{\ell+1}\right)=\ell$ para todo $\lambda \in\left(\lambda_{\ell}, \lambda_{\ell+1}\right)$.

Demonstração. Para $\lambda \in\left(\lambda_{\ell}, \lambda_{\ell+1}\right)$, temos $0<n(1+\epsilon)\left(\lambda-\lambda_{\ell}\right)<1$ e

$$
\begin{aligned}
h(\lambda) & =\lceil n \lambda(1+\epsilon)\rceil-1=\left\lceil n \lambda_{\ell}(1+\epsilon)+n(1+\epsilon)\left(\lambda-\lambda_{\ell}\right)\right\rceil-1= \\
& =\left\lceil n\left[\frac{\ell}{n}+(1+\epsilon)\left(\lambda-\lambda_{\ell}\right)\right]\right\rceil-1=\ell-1+\left\lceil n(1+\epsilon)\left(\lambda-\lambda_{\ell}\right)\right\rceil= \\
& =\ell=\left\lceil n\left[\frac{\ell+1}{n(1+\epsilon)}(1+\epsilon)\right]\right\rceil-1=h\left(\lambda_{\ell+1}\right) .
\end{aligned}
$$

Lema C.2 (Chen, 2011). Seja $\lambda_{\ell}=\ell /(n(1-\epsilon))$ em que $\ell \in \mathbb{Z}$. Então, $g(\lambda)=g\left(\lambda_{\ell}\right)=\ell+1$ para todo $\lambda \in\left(\lambda_{\ell}, \lambda_{\ell+1}\right)$.

Demonstração. Para $\lambda \in\left(\lambda_{\ell}, \lambda_{\ell+1}\right)$, temos $-1<n(1-\epsilon)\left(\lambda-\lambda_{\ell+1}\right)<0$ e

$$
\begin{aligned}
g(\lambda) & =\lfloor n \lambda(1-\epsilon)\rfloor+1=\left\lfloor n\left[\lambda_{\ell+1}(1-\epsilon)=(1-\epsilon)\left(\lambda-\lambda_{\ell+1}\right)\right]\right\rfloor+1= \\
& =\left\lfloor n \frac{\ell+1}{n(1-\epsilon)}(1-\epsilon)\right\rfloor+\left\lfloor n(1-\epsilon)\left(\lambda-\lambda_{\ell+1}\right)\right\rfloor+1=\left\lfloor n \frac{\ell+1}{n(1-\epsilon)}(1-\epsilon)\right\rfloor-1+1= \\
& =\ell+1=\left\lfloor n \frac{\ell}{n(1-\epsilon)}(1-\epsilon)\right\rfloor+1=g\left(\lambda_{\ell}\right) .
\end{aligned}
$$


Lema C.3 (Chen, 2011). Sejam $\alpha<\beta$ dois elementos consecutivos do arranjo ascendente de todos os elementos distintos de $\{a, b\} \cup\{\ell /(n(1-\epsilon)) \in(a, b): \ell \in \mathbb{Z}\} \cup\{\ell /(n(1+\epsilon)) \in(a, b): \ell \in \mathbb{Z}\}$. Então, $g(\lambda)$ e $h(\lambda)$ são constantes para qualquer $\lambda \in(\alpha, \beta)$.

Demonstração. Uma vez que $\alpha$ e $\beta$ são dois elementos consecutivos do arranjo ascendente de todos os elementos distintos do conjunto, não há inteiro $\ell$ tal que $\alpha<\ell /(n(1-\epsilon))<\beta$ or $\alpha<\ell /(n(1+\epsilon))<$ $\beta$. Logo existem dois inteiros $\ell$ e $\ell^{\prime}$ tais que $(\alpha, \beta) \subseteq(\ell /(n(1-\epsilon)),(\ell+1) /(n(1-\epsilon)))$ e $(\alpha, \beta) \subseteq$ $\left(\ell^{\prime} /(n(1+\epsilon)),\left(\ell^{\prime}+1\right) /(n(1+\epsilon))\right)$. Aplicando os Lemas C.1 e C.2, temos $g(\lambda)=g(\ell /(n(1-\epsilon)))$ e $h(\lambda)=h\left(\left(\ell^{\prime}+1\right) /(n(1+\epsilon))\right)$ para todo $\lambda \in(\alpha, \beta)$.

Lema C.4 (Chen, 2011). Para todo $\lambda \in(0, \infty), \lim _{\eta \rightarrow 0} C(\lambda+\eta) \geq C(\lambda)$ e $\lim _{\eta \rightarrow 0} C(\lambda-\eta) \geq C(\lambda)$.

Demonstração. Observemos que $h(\lambda+\eta) \geq h(\lambda)$ para todo $\eta>0$ e que

$$
\begin{aligned}
g(\lambda+\eta) & =\lfloor n(\lambda+\eta)(1-\epsilon)\rfloor+1=\lfloor n \lambda(1-\epsilon)\rfloor+1+\lfloor n \lambda(1-\epsilon)-\lfloor n \lambda(1-\epsilon)\rfloor+n \eta(1-\epsilon)\rfloor= \\
& =\lfloor n \lambda(1-\epsilon)\rfloor+1=g(\lambda)
\end{aligned}
$$

para $0<\eta<\{1+\lfloor n \lambda(1-\epsilon)\rfloor-n \lambda(1-\epsilon)\} /(n(1-\epsilon))$. Então

$$
S(n(\lambda+\eta), g(\lambda+\eta), h(\lambda+\eta), n k) \geq S(n(\lambda+\eta), g(\lambda), h(\lambda), n k)
$$

para $0<\eta<\{1+\lfloor n \lambda(1-\epsilon)\rfloor-n \lambda(1-\epsilon)\} /(n(1-\epsilon))$. Como

$$
h(\lambda+\eta)=\lceil n(\lambda+\eta)(1+\epsilon)\rceil-1=\lceil n \lambda(1+\epsilon)\rceil-1+\lceil n \lambda(1+\epsilon)-\lceil n \lambda(1+\epsilon)\rceil+n \eta(1+\epsilon)\rceil,
$$

temos

$$
h(\lambda+\eta)=\left\{\begin{array}{lllll}
\lceil n \lambda(1+\epsilon)\rceil & \text { para } & n \lambda(1+\epsilon)=\lceil n \lambda(1+\epsilon)\rceil & \text { e } & 0<\eta<1 /(n(1+\epsilon)) \\
\lceil n \lambda(1+\epsilon)\rceil-1 & \text { para } & n \lambda(1+\epsilon) \neq\lceil n \lambda(1+\epsilon)\rceil & \text { e } & 0<\eta<\frac{\lceil n \lambda(1+\epsilon)\rceil-n \lambda(1+\epsilon)}{n(1+\epsilon)} .
\end{array}\right.
$$

Logo $g(\lambda+\eta)$ e $h(\lambda+\eta)$ são independente de $\eta$ se $\eta>0$ é pequeno o bastante. $S(n(\lambda+\eta), g, h, n k)$ é contínua em relação a $\eta$ para $g$ e $h$ fixados, o $\lim _{\eta \rightarrow 0} S(n(\lambda+\eta), g(\lambda+\eta), h(\lambda+\eta), n k)$ existe. Assim temos que

$$
\begin{aligned}
\lim _{\eta \rightarrow 0} C(\lambda+\eta) & =\lim _{\eta \rightarrow 0} S(n(\lambda+\eta), g(\lambda+\eta), h(\lambda+\eta), n k) \geq \lim _{\eta \rightarrow 0} S(n(\lambda+\eta), g(\lambda), h(\lambda), n k)= \\
& =S(n \lambda, g(\lambda), h(\lambda), n k)=C(\lambda)
\end{aligned}
$$

em que a desigualdade segue de (C.2). Observemos que $g(\lambda-\eta) \leq g(\lambda)$ para todo $\eta>0$ e que

$$
\begin{aligned}
h(\lambda-\eta) & =\lceil n(\lambda-\eta)(1+\epsilon)\rceil-1=\lceil n \lambda(1+\epsilon)\rceil-1+\lceil n \lambda(1+\epsilon)-\lceil n \lambda(1+\epsilon)\rceil-n \eta(1+\epsilon)\rceil= \\
& =\lceil n \lambda(1+\epsilon)\rceil-1=h(\lambda)
\end{aligned}
$$

para $0<\eta<\{1+n \lambda(1+\epsilon)-\lceil n \lambda(1+\epsilon)\rceil\} /(n(1+\epsilon))$. Então

$$
S(n(\lambda-\eta), g(\lambda-\eta), h(\lambda-\eta), n k) \geq S(n(\lambda-\eta), g(\lambda), h(\lambda), n k)
$$

para $0<\eta<\{1+n \lambda(1+\epsilon)-\lceil n \lambda(1+\epsilon)\rceil\} / n(1+\epsilon)$. Como

$$
g(\lambda-\eta)=\lfloor n(\lambda-\eta)(1-\epsilon)\rfloor+1=\lfloor n \lambda(1-\epsilon)\rfloor+1+\lfloor n \lambda(1-\epsilon)-\lfloor n \lambda(1-\epsilon)\rfloor-n \eta(1-\epsilon)\rfloor,
$$

temos

$$
g(\lambda-\eta)=\left\{\begin{array}{lllll}
\lfloor n \lambda(1-\epsilon)\rfloor & \text { para } & n \lambda(1-\epsilon)=\lfloor n \lambda(1-\epsilon)\rfloor & \text { e } & 0<\eta<1 /(n(1-\epsilon)) \\
\lfloor n \lambda(1-\epsilon)\rfloor+1 & \text { para } & n \lambda(1-\epsilon) \neq\lfloor n \lambda(1-\epsilon)\rfloor & \text { e } & 0<\eta<\frac{n \lambda(1-\epsilon)-\lfloor n \lambda(1-\epsilon)\rfloor}{n(1-\epsilon)} .
\end{array}\right.
$$


Logo $g(\lambda-\eta)$ e $h(\lambda-\eta)$ são independentes de $\eta$ se $\eta>0$ é pequeno o bastante. Uma vez que $S(n, g, h, \lambda-\eta)$ é contínua com relação a $\eta$ para $g$ e $h$ fixos, o $\lim _{\eta \rightarrow 0} S(n(\lambda-\eta), g(\lambda-\eta), h(\lambda-\eta), n k)$ existe. Então

$$
\begin{aligned}
\lim _{\eta \rightarrow 0} C(\lambda-\eta) & =\lim _{\eta \rightarrow 0} S(n(\lambda-\eta), g(\lambda-\eta), h(\lambda-\eta), n k) \geq \lim _{\eta \rightarrow 0} S(n(\lambda-\eta), g(\lambda), h(\lambda), n k)= \\
& =S(n \lambda, g(\lambda), h(\lambda), n k)=C(\lambda)
\end{aligned}
$$

em que a desigualdade segue de (C.3).

Lema C.5 (Chen, 2011). Sejam $\alpha<\beta$ dois elementos consecutivos do arranjo ascendente de todos os elementos distintos de $\{a, b\} \cup\{\ell /(n(1-\epsilon)) \in(a, b): \ell \in \mathbb{Z}\} \cup\{\ell /(n(1+\epsilon)) \in(a, b): \ell \in \mathbb{Z}\}$. Então, $C(\lambda) \geq \min \{C(\alpha), C(\beta)\}$ para todo $\lambda \in(\alpha, \beta)$.

Demonstração. A demonstração deste Lema é similar a demonstração do Lema B.7.

A afirmação sobre o mínimo da probabilidade de cobertura segue imediatamente do Lema C.5. O número de elementos do conjunto discreto descrito no Teorema 3.4 pode ser calculado como indicado em Chen (2011). Como

$$
a<\ell /(n(1+\epsilon))<b \Leftrightarrow 1+\lfloor n a(1+\epsilon)\rfloor \leq \ell \leq\lceil n b(1+\epsilon)\rceil-1,
$$

o número de elementos em $\{\ell /(n(1+\epsilon)) \in(a, b): \ell \in \mathbb{Z}\}$ é

$$
\lceil n b(1+\epsilon)\rceil-\lfloor n a(1+\epsilon)\rfloor-1<n b(1+\epsilon)+1-[n a(1+\epsilon)-1]-1=n(b-a)(1+\epsilon)+1 .
$$

Por outro lado,

$$
a<\ell /(n(1-\epsilon))<b \Leftrightarrow 1+\lfloor n a(1-\epsilon)\rfloor \leq \ell \leq\lceil n b(1-\epsilon)\rceil-1,
$$

e o número de elementos em $\{\ell /(n(1-\epsilon)) \in(a, b): \ell \in \mathbb{Z}\}$ é

$$
\lceil n b(1-\epsilon)\rceil-\lfloor n a(1-\epsilon)\rfloor-1<n b(1-\epsilon)+1-[n a(1-\epsilon)-1]-1=n(b-a)(1-\epsilon)+1 .
$$

Consequentemente, o número total de elementos no conjunto $\{a, b\} \cup\{\ell /(n(1-\epsilon)) \in(a, b): \ell \in$ $\mathbb{Z}\} \cup\{\ell /(n(1+\epsilon)) \in(a, b): \ell \in \mathbb{Z}\}$ é menor que $2 n(b-a)+4$, o que completa a demonstração.

\section{C.2 Demonstração do Teorema 3.5}

Primeiramente notemos que

$$
\mathbb{P}\left[|\widehat{\lambda}-\lambda|<\epsilon_{a} \text { ou }|\widehat{\lambda}-\lambda| / \lambda<\epsilon_{r}\right]= \begin{cases}\mathbb{P}\left[|\widehat{\lambda}-\lambda|<\epsilon_{a}\right], & \text { se } \quad \lambda \in\left[a, \epsilon_{a} / \epsilon_{r}\right], \\ \mathbb{P}\left[|\widehat{\lambda}-\lambda| / \lambda<\epsilon_{r}\right], & \text { se } \quad \lambda \in\left(\epsilon_{a} / \epsilon_{r}, b\right] .\end{cases}
$$

Logo o resultado segue dos Teoremas 3.3 e 3.4. 
APÊNDICE C 


\section{Apêndice D}

\section{Pseudo-algoritmos}

Todos os pseudo-algoritmos apresentados neste apêndice foram implementados no software R e podem ser obtidos na página www.ime.usp.br/ eliardo

\section{D.1 Tamanho amostral para o teste (2.4)}

Considere $S=\sum_{i=1}^{n} X_{i}$. Para calcular o menor tamanho amostral $n$ para o qual o poder do teste (2.4) é $\geq 1-b$ e a significância é $\leq a$, com $a$ e $b$ fixados, e considerando alíquotas de $v m^{3}$ execute os seguintes passos.

1. Fixe o valor de $a, b, v$ e $\lambda_{1}$ tal que $\lambda_{1}>10$;

2. Faça $n=2$;

3. Calcule $c$ tal que $a=\mathbb{P}[S \geq c \mid \lambda=10]$ por meio de (2.6);

4. Calcule a probabilidade $\beta\left(\lambda_{1}\right)=\mathbb{P}\left[S<c \mid \lambda=\lambda_{1}\right]$ utilizando (2.6);

5. Se $1-\beta\left(\lambda_{1}\right) \geq 1-b$, pare o processo. O valor $n$ obtido nesse passo é o valor desejado. Em caso contrário, faça $n=n+1$ e volte para 3 .

\section{D.2 Tamanho amostral para o teste (2.7)}

Considere $S_{e}=\sum_{h=1}^{L} X_{h}$. Para calcular os menores volumes amostrais $\left(v_{h}\right)$ para o qual o poder do teste (2.7) é $\geq 1-b$ e a significância é $\leq a$, com $a$ e $b$ fixados, e considerando alíquotas de $v m^{3}$ execute os seguintes passos.

1. Fixe o valor de $a, b$ e $v$;

2. Fixe os valores $V_{h}$ e $\lambda_{h}(h=1,2, \ldots, L)$ tais que $\lambda_{1}=(1 / V) \sum_{h=1}^{L} \lambda_{h} V_{h}>10$, em que $V=\sum_{h=1}^{L} V_{h}$

3. Faça $n=2$;

4. Calcule $c$ tal que $a=\mathbb{P}\left[S_{e} \geq c \mid \lambda=10\right]$ utilizando (2.8);

5. Calcule a probabilidade $\beta\left(\lambda_{1}\right)=\mathbb{P}\left[S_{e}<c \mid \lambda=\lambda_{1}\right]$ utilizando (2.8);

6. Se $1-\beta\left(\lambda_{1}\right) \geq 1-b$, pare o processo. O valor $v$ obtido nesse passo é o valor desejado. Em caso contrário, faça $v=v+0.001$ e volte para 4 .

Por meio de $v$ calcula-se o volume amostral para cada estrato $(h)$ utilizando $v_{h}=\left(V_{h} / V\right) v$. Nesta dissertação foi usado 0.001 como incremento de $v$ no passo 6 do algoritmo devido à magnitude dos valores de $V_{h}$, porém outro valor pode ser utilizado dependendo do problema em estudo. 


\section{D.3 Tamanho amostral para o teste (2.15)}

Considere $S=\sum_{i=1}^{n} X_{i}$. Para calcular o menor tamanho amostral $n$ para o qual o poder do teste (2.4) é $\geq 1-b$ e a significância é $\leq a$, com $a$ e $b$ fixados, e considerando alíquotas de $v m^{3}$ execute os seguintes passos.

1. Fixe o valor de $a, b, k$ e $\lambda_{1}$ tal que $\lambda_{1}>10$;

2. Faça $n=2$;

3. Calcule $c$ tal que $a=\mathbb{P}[S \geq c \mid \lambda=10]$ por meio de (2.16);

4. Calcule a probabilidade $\beta\left(\lambda_{1}\right)=\mathbb{P}\left[S<c \mid \lambda=\lambda_{1}\right]$ utilizando (2.16);

5. Se $1-\beta\left(\lambda_{1}\right) \geq 1-b$, pare o processo. O valor $n$ obtido nesse passo é o valor desejado. Em caso contrário, faça $n=n+1$ e volte para 3 .

\section{D.4 Tamanho amostral exato para o Teorema 3.3}

Seja o conjunto $\mathcal{S}=\{a, b\} \cup\left\{\ell / n+\epsilon_{a} \in(a, b): \ell \in \mathbb{Z}\right\} \cup\left\{\ell / n-\epsilon_{a} \in(a, b): \ell \in \mathbb{Z}\right\} \operatorname{com} a, b, k$ e $\epsilon_{a}$ fixados. Para calcular $n$ execute os seguintes passos.

1. Fixe o nível de confiança $1-\delta$;

2. Faça $n=2$;

3. Calcule a probabilidade de cobertura, $C(\lambda)$ (B.1), para todos os elementos do conjunto $\mathcal{S}$ utilizando (2.16);

4. Obtenha o mínimo dessas probabilidades e se esse valor for maior que $1-\delta$, pare o processo. $\mathrm{O}$ valor $n$ obtido nesse processo é o valor desejado. Em caso contrário, faça $n=n+1$ e volte para 3.

\section{D.5 Tamanho amostral exato para o Teorema 3.4}

Seja o conjunto $\mathcal{S}=\{a, b\} \cup\left\{\ell /\left(n\left(1-\epsilon_{r}\right)\right) \in(a, b): \ell \in \mathbb{Z}\right\} \cup\left\{\ell /\left(n\left(1+\epsilon_{r}\right)\right) \in(a, b): \ell \in \mathbb{Z}\right\}$ com $a, b, k$ e $\epsilon_{r}$ fixados. Para calcular $n$ execute os seguintes passos.

1. Fixe o nível de confiança $1-\delta$;

2. Faça $n=2$;

3. Calcule a probabilidade de cobertura, $C(\lambda)$ (C.1), para todos os elementos do conjunto $\mathcal{S}$ utilizando (2.16);

4. Obtenha o mínimo dessas probabilidades e se esse valor for maior que $1-\delta$, pare o processo. O valor $n$ obtido nesse processo é o valor desejado. Em caso contrário, faça $n=n+1$ e volte para 3.

\section{D.6 Tamanho amostral exato para o Teorema 3.5}

Aplique conjuntamente o pseudo-algoritmo D.4 considerando os limites $a$ e $b=\epsilon_{a} / \epsilon_{r}$, e o pseudo-algoritmo D.5 considerando os limites $a=\epsilon_{a} / \epsilon_{r}$ e $b$. 


\section{Referências}

Basawa, I. V. \& RAO, B. L. S. (1980). Statistical inference for stochastic processes. London: Academic Press. 11

Basurko, O. C. \& Mesbahi, E. (2011). Statistical representativeness of ballast water sampling. Em Proceedings of the Institution of Mechanical Engineer, Part M. Journal of Engineering for the Maritime Environment, 183-190. 3, 5, 41

Beals, R. \& Wong, R. (2012). Special functions: A graduate text. Cambridge: Cambridge University Press. 51

Beasley, C. R., Tagliaro, C. H. \& Figueiredo, W. B. (2003). The occurrence of the asian clam corbicula fluminea in lower amazon basin. Acta Amazônica 33, 317-324. 1

Bierman, S. M., VRies, P. \& KaAg, N. H. B. M. (2012). The development of a full standard methodology for testing ballast water discharges for gross non-compliance of the IMO's Ballast Water Management Convention (EMSA/NEG/12/2012). Relatório técnico C124/12, IMARES Wageningen UR. 21, 42

Broyden, C. G. (1970). The convergence of a class of double-rank minimization algorithms. Journal of the Institute of Mathematics and Its Applications 6, 76-90. 12, 31

Carlton, J. T. (2001). Introduced species in U.S. coastal waters: Environmental impacts and management priorities. Pew Oceans Commission. Arlington, Virginia. 1

Carmo, M. C. (2008). Plano de gestão de água de lastro de embarcações. Dissertação de mestrado. Instituto Militar de Engenharia, Rio de Janeiro. 1

Carney, K. J., Basurko, O. C., Pazouki, K., Marsham, S., Delany, J. E., DeSaI, D. V., Anil, A. C. \& Mesbahi, E. (2013). Difficulties in obtaining representative samples for compliance with the Ballast Water Management Convention. http://dx.doi.org/10.1016/j.marpolbul.2012.12.016. 2

Carqueija, C. R. G. \& GouvêA, E. P. (1996). A ocorrência na costa brasileira de um Portunidae (Crustacea, Decapoda) originário do indo-pacífico e mediterrâneo. Nauplius 4, 105-112. 1

Chen, X. (2007). Exact computation of minimum sample size for estimation of Poisson parameters. http://arxiv.org/abs/0707.2116. 27, 28

Chen, X. (2008). A simple sample size formula for estimating means of Poisson random variables. http://arxiv.org/abs/0804.3033. 26

Chen, X. (2011). Exact computation of minimum sample size for estimation of binomial parameters. Journal of Statistical Planning and Inference 141, 2622-2632. 27, 28, 29, 49, 53, 55, 57

David, M., Gollasch, S., Cabrini, M., Perkovič, M., Bosnjak, D. \& Virgilio, D. (2007). Results from the first ballast water sampling study in the Mediterranean Sea, the Port of Koper study. Marine Pollution Bulletin 54, 53-65. 1 
DeGroot, M. H. (1975). Probability and statistics. Menlo Park: Addison-Wesley Publishing Company. 22

Fletcher, R. (1970). A new approach to variable metric algorithms. Computer Journal 13, 317-322. 12,31

Goldfarb, D. (1970). A family of variable metric updates derived by variational means. Mathematics of Computation 24, 23-26. 12, 31

Hager, A. X. (2008). Identificação genética de espécies exóticas invasoras do filo mollusca nos rios Tapajós e Amazonas, mesorregião do baixo Amazonas. Dissertação de mestrado. Universidade Federal do Pará, Santarém. 1

Haight, F. A. (1967). Handbook of the Poisson distribution. New York: John Wiley \& Sons Inc. 5,42

IMO (2008). Report of the Marine Environment Protection Committee, Annex 3: Resolution MEPC 173(58) - Guidelines for ballast water sampling (G2). Relatório técnico MEPC 58/23, IMO, London. 2, 41

IMO/MEPC (1998). Harmful aquatic organisms in ballast water, report of the working group on ballast water convened during MEPC 41. Relatório técnico MEPC 42/8, IMO. 1

Mansfield, B. (2012). 'Law of the sea - Protecting the oceans', Te Ara - the Encyclopedia of New Zealand. 2

McCarthy, S. A., Mcphearson, R. M., Guarino, A. \& Gaines, J. (1992). Toxigenic Vibrio cholerae 01 and cargo ships entering Gulf of Mexico. The Lancet 339, 624-625. 1

Miller, A. W., Frazier, M., Smith, G. E., Perry, E. S., Ruiz, G. M. \& Tamburri, M. N. (2011). Enumerating sparse organisms in ships' ballast water: Why counting to 10 is not easy. Environmental Science and Technology 45, 3539-3546. 3, 5, 6, 9, 41

Murphy, K. R., Ritz, D. \& Hewitt, C. L. (2002). Heterogeneous zooplankton distribution in a ship's ballast tanks. Journal of Plankton Research 24, 729-734. 3, 9

National Ballast Information Clearinghouse (2009). NBIC Online Database. Electronic publication, Smithsonian Environmental Research Center \& United States Coast Guard. 3

Pereira, N. N. (2012). Alternativas de tratamento da água de lastro em portos exportadores de minério de ferro. Tese de doutorado. Escola Politécnica, Universidade de São Paulo, São Paulo. 2,4

R Development Core Team (2012). R: A Language and Environment for Statistical Computing. R Foundation for Statistical Computing, Vienna, Austria. ISBN 3-900051-07-0, URL http://www.R-project.org/. 12, 31

Ross, S. M. (1996). Stochastic processes. New York: John Wiley \& Sons. 11

Roussas, G. G. (1997). A course in mathematical statistics, $2^{\mathrm{a}}$ ed. San Diego: Academic Press. 11

Ruiz, G. M., Rawlings, T. K., Dobbs, F. C., Drake, L. A., Mullady, T., Huq, A. \& Colwell, R. R. (2000). Global spread of microorganisms by ships: Ballast water discharged from vessels harbours a cocktail of potential pathogens. Nature 408, 49-50. 1

Sen, P. K., Singer, J. M. \& De Lima, A. C. P. (2009). From Finite Sample to Asymptotic Methods in Statistics. Cambridge: Cambridge University Press. 11, 31 
Shanno, D. F. (1970). Conditioning of quasi-newton methods for function minimization. Mathematics of Computation 24, 647-656. 12, 31

Souza, R. C. C. L., Fernandes, F. C., Danelon, O. M., Larsen, K. T. S., Silva, J. S. V., Collichio, F. \& Rapagnã, L. (2001). Metodologia de amostragem dos organismos transportados em água de lastro dos navios mercantes. Pesquisa Naval 14, 221-236. 1

Tavares, M. \& Mendonça JR., J. B. (2004). Introdução de crustáceos decápodes exóticos no brasil: Uma roleta ecológica. Em J. S. V. Silva \& R. C. C. L. Souza, eds., Água de lastro e Bioinvasão. Interciência. 1

Walton, H. P. (1998). Synthesizing science and policy to reduce the probability of marine biological invasions due to ballast water discharge in the Port of Morehead city. Dissertação de mestrado. Universidade Duke, Durham. 1 